\title{
HOMOLOGY AND STANDARD CONSTRUCTIONS
}

\author{
MICHAEL BARR AND JON BECK
}

\section{Introduction}

In ordinary homological algebra, if $M$ is an $R$-module, the usual way of starting to construct a projective resolution of $M$ is to let $F$ be the free $R$-module generated by the elements of $M$ and $F \rightarrow M$ the epimorphism determined by $(m) \mapsto m$. One then takes the kernel of $F \rightarrow M$ and continues the process. But notice that in the construction of $F \rightarrow M$ a lot of structure is customarily overlooked. $F$ is actually a functor $M G$ of $M$, $F \rightarrow M$ is an instance of a natural transformation $G \rightarrow$ (identity functor); there is also a "comultiplication" $G \rightarrow G G$ which is a little less evident. The functor $G$, equipped with these structures, is an example of what is called a standard construction or "cotriple".

In this paper we start with a category $\mathbf{C}$, a cotriple $\mathbf{G}$ in $\mathbf{C}$, and show how resolutions and derived functors or homology can be constructed by means of this tool alone. The category $\mathbf{C}$ will be non-abelian in general (note that even for modules the cotriple employed fails to respect the additive structure of the category), and the coefficients will consist of an arbitrary functor $E: \mathbf{C} \rightarrow \mathscr{A}$, where $\mathscr{A}$ is an abelian category. For ordinary homology and cohomology theories, $E$ will be tensoring, homming or deriving with or into a module of some kind.

To summarize the contents of the paper: In Section 1 we define the derived functors and give several examples of categories with cotriples. In Section 2 we study the derived functors $H_{n}(, E)_{\mathbf{G}}$ as functors on $\mathbf{C}$ and give several of their properties. In Section 3 we fix a first variable $X \in \mathbf{C}$ and study $H_{n}(X,)_{\mathbf{G}}$ as a functor of the abelian variable $E$. As such it admits a simple axiomatic characterization. Section 4 considers the case in which $\mathbf{C}$ is additive and shows that the general theory can always, in effect, be reduced to that case. In Section 5 we study the relation between cotriples and projective classes (defined - essentially - by Eilenberg-Moore [Eilenberg \& Moore (1965)]) and show that the homology only depends on the projective class defined by the cotriple. Sections 6-9 are concerned largely with various special properties that these derived functors possess in well known algebraic categories (groups, modules, algebras, ...). In Section 10 we consider the problem of defining a cotriple to produce a given projective class (in a sense, the converse problem to that studied in Section 5) by means of "models". We also compare the results with other theories of derived functors based on models. Section 11 is concerned with some technical items on acyclic models.

The first author is partially supported by NSF Grant GP 5478 and the second has been supported by an NAS-NRC Postdoctoral Fellowship

(C) Michael Barr and Jon Beck, . Permission to copy for private use granted. 
Before beginning the actual homology theory, we give some basic definitions concerning the simplicial objects which will be used. Let $\mathbf{G}=(G, \varepsilon, \delta)$ be a cotriple in $\mathbf{C}$, that is,

$$
\begin{gathered}
\mathbf{C} \stackrel{G}{\longrightarrow} \mathbf{C} \\
G \stackrel{\varepsilon}{\longrightarrow} \mathbf{C} \quad \text { and } \quad G \stackrel{\delta}{\longrightarrow} G G
\end{gathered}
$$

and the unitary and associative laws hold, as given in the Introduction to this volume. (Note that here and throughout we identify identity maps with the corresponding objects; thus $\mathbf{C}$ denotes the identity functor $\mathbf{C} \rightarrow \mathbf{C}$.) If $X$ is an object in $\mathbf{C}$, the following is an augmented simplicial object in $\mathbf{C}$ :

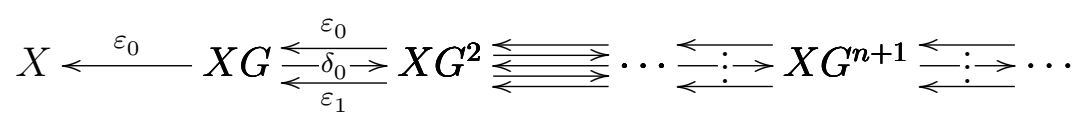

$X G^{n+1}$ is the $n$-dimensional component,

$$
\varepsilon_{i}=G^{i} \varepsilon G^{n-i}: G^{n+1} \rightarrow G^{n} \text { and } \delta_{i}=G^{i} \delta G^{n-i}: G^{n+1} \rightarrow G^{n+2}
$$

for $0 \leq i \leq n$, and the usual simplicial identities hold:

$$
\begin{array}{ll}
\varepsilon_{i} \varepsilon_{j}=\varepsilon_{j+1} \varepsilon_{i} & \text { for } i \leq j \\
\delta_{i} \delta_{j}=\delta_{j-1} \delta_{i} & \text { for } i<j
\end{array} \quad \delta_{i} \varepsilon_{j}= \begin{cases}\varepsilon_{j-1} \delta_{i} & \text { for } i<j-1 \\
\text { identity } & \text { for } i=j-1 \text { and } i=j \\
\varepsilon_{j} \delta_{i-1} & \text { for } i>j .\end{cases}
$$

(composition is from left to right).

If $X$ admits a map $s: X \rightarrow X G$ such that $s \circ X \varepsilon=X$ ( $\operatorname{such} X$ are called $\mathbf{G}$-projective, see (2.1)), then the above simplicial object develops a contraction

$$
X \stackrel{h_{-1}}{\longrightarrow} X G \stackrel{h_{0}}{\longrightarrow} X G^{2} \longrightarrow \cdots \longrightarrow X G^{n+1} \stackrel{h_{n}}{\longrightarrow} \cdots
$$

namely $h_{n}=s G^{n+1}$. These operators satisfy the equations

$$
h_{n} \varepsilon_{0}=X G^{n+1} \quad \text { and } \quad h_{n} \varepsilon_{i}=\varepsilon_{i-1} h_{n-1}
$$

for $0<i \leq n+1$ and $n \geq-1$. They express the fact that the simplicial object $\left(X G^{n+1}\right)_{n \geq 0}$ is homotopically equivalent to the constant simplicial object which has $X$ in all dimensions.

If $\left(X_{n}\right)_{n \geq-1}$ is a simplicial set with such a contraction, we conclude $\Pi_{n}(X)=0$ for $n>0$, and $\Pi_{0}(X)=X_{-1}$.

On the other hand, if $E: \mathbf{C} \rightarrow \mathscr{A}$ is a functor into any other category and $E$ possesses a natural transformation $\vartheta: E \rightarrow G E$ such that $\vartheta \circ \varepsilon E=E$, then $\left(X G^{n+1} E\right)_{n \geq-1}$ also has a contraction

$$
X E \stackrel{h_{-1}}{\longrightarrow} X G E \stackrel{h_{0}}{\longrightarrow} X G^{2} E \longrightarrow \cdots \longrightarrow X G^{n+1} E \stackrel{h_{n}}{\longrightarrow} \cdots
$$


Here $h_{n}=X G^{n+1} \vartheta$ and the identities satisfied are a little different (This is a "right" homotopy [Kleisli (1967)]):

$$
h_{n} \varepsilon_{i}=\varepsilon_{i} h_{n-1} \quad \text { and } \quad h_{n} \varepsilon_{n+1}=X G^{n+1} E
$$

for $0 \leq i \leq n$ and $n \geq-1$. Both here and above some equations involving degeneracies also hold, but our concern is usually with homology so we omit them.

If the functor $E$ takes values in an abelian category, then as follows from a well known theorem of J.C. Moore [Moore (1956)] the homotopy in any sense of $\left(X G^{n+1} E\right)_{n \geq 0}$ is the same as the homology of the associated chain complex

$$
0 \longleftarrow X G E \longleftarrow \partial_{1} X G^{2} E \longleftarrow \stackrel{\partial_{2}}{\longleftarrow} \cdots \stackrel{\partial_{n}}{\longleftarrow} X G^{n+1} E \longleftarrow \cdots
$$

where $\partial_{n}=\sum(-1)^{i} \varepsilon_{i} E$. If there is a contraction, $H_{n}=0$ for $n>0, H_{0}=X E$.

\section{Definition of the homology theory $H_{n}(X, E)_{\mathbf{G}}$}

Let $X \in \mathbf{C}$, let $\mathbf{G}=(G, \varepsilon, \delta)$ be a cotriple in $\mathbf{C}$, and let $E: \mathbf{C} \rightarrow \mathscr{A}$ be a functor into an abelian category. Applying $E$ to $\left(X G^{n+1}\right)_{n \geq-1}$ we get an augmented simplicial object in $\mathscr{A}$ :

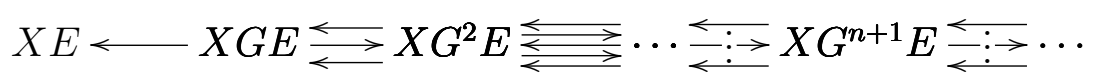

The homotopy of this simplicial object, or what is the same thing by Moore's theorem, the homology of the associated chain complex

$$
0 \longleftarrow X G E \longleftarrow \partial_{1} G^{2} E \longleftarrow \partial_{2}
$$

is denoted by $H_{n}(X, E)_{\mathbf{G}}$, for $n \geq 0$. These are the homology groups (objects) of $X$ with coefficients in $E$ relative to the cotriple $\mathbf{G}$. Often $\mathbf{G}$ is omitted from the notation if it is clear from the context.

The homology is functorial with respect to maps $X \rightarrow X_{1}$ in $\mathbf{C}$ and natural transformations of the coefficient functors $E \rightarrow E_{1}$.

A natural transformation (augmentation)

$$
H_{0}(, E)_{\mathbf{G}} \stackrel{\lambda=\lambda E}{\longrightarrow} E
$$

is defined by the fact that $H_{0}$ is a cokernel:

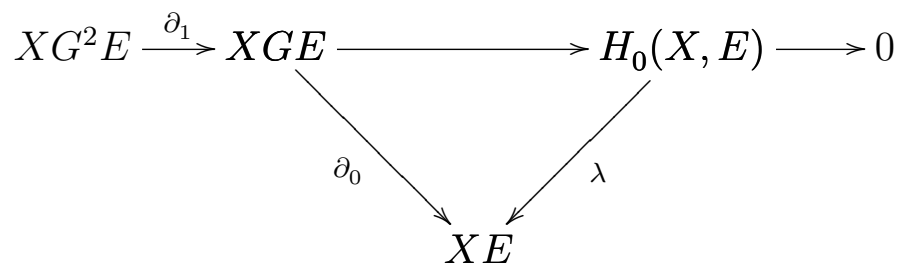


$\lambda\left(H_{0}(, E)\right)$ and $H_{0}(, \lambda E)$ coincide since they both fit in the diagram

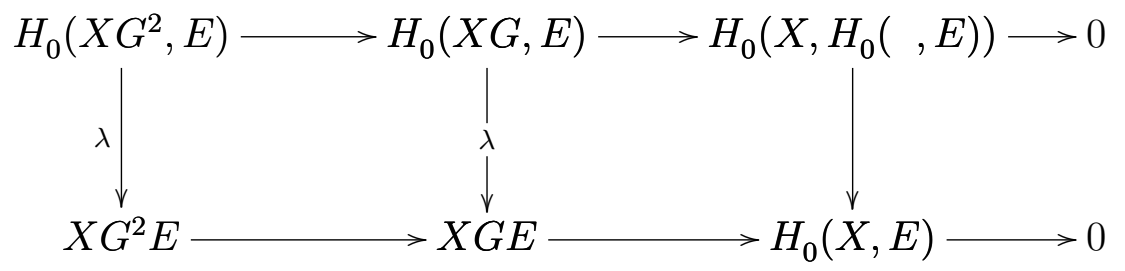

Thus $\lambda$ can be viewed as a reflection into the subcategory of all functors $E: \mathbf{C} \rightarrow \mathscr{A}$ with $\lambda: H_{0}(, E) \stackrel{\cong}{\longrightarrow} E$. These are the functors which transform $X G^{2} \Longrightarrow X G \longrightarrow X$ into a coequalizer diagram in $\mathscr{A}$, for all $X \in \mathbf{C}$, a sort of right exactness property.

The following variations occur. If, dually, $\mathbf{T}=(T, \eta, \mu)$ is a triple in $\mathbf{C}$ and $E: \mathbf{C} \rightarrow \mathscr{A}$ is a coefficient functor, cohomology groups $H^{n}(X, E)_{\mathbf{T}}$, for $n \geq 0$, are defined by means of the cochain complex

$$
0 \longrightarrow X T E \stackrel{d^{1}}{\longrightarrow} X T^{2} E \stackrel{d^{2}}{\longrightarrow} \cdots \longrightarrow X T^{n+1} E \stackrel{d^{n}}{\longrightarrow} \cdots
$$

where $d^{n}=\sum(-1)^{i} X \eta_{i} E$ for $0 \leq i \leq n$, and $\eta_{i}=T^{i} \eta T^{n-i}$.

If $\mathbf{G}=(G, \varepsilon, \delta)$ is a cotriple and $E: \mathbf{C}^{\text {op }} \rightarrow \mathscr{A}$ is a functor (or $E: \mathbf{C} \rightarrow \mathscr{A}$ is contravariant), the complex would take the form

$$
0 \longrightarrow X G E \longrightarrow X G^{2} E \longrightarrow \cdots \longrightarrow X G^{n+1} E \longrightarrow \cdots
$$

In effect this is cohomology with respect to the triple $\mathbf{G}^{\text {op }}$ in the dual category. However, we write the theory as $H^{n}(X, E)_{\mathbf{G}}$.

For the most part we will only state theorems about the cotriple-covariant functor situation and leave duals to the reader. Usually cotriples arise from adjoint functors, although another method of construction will be essayed in Section 10. If $F: \mathbf{A} \rightarrow \mathbf{C}$ is left adjoint to $U: \mathbf{C} \rightarrow \mathbf{A}$, there are well know natural transformations $\eta: \mathbf{A} \rightarrow F U$ and $\varepsilon: U F \rightarrow \mathbf{C}$. If we set $G=U F$, we have $\varepsilon: G \rightarrow \mathbf{C}$, and if we set $\delta=U \eta F$, then $\delta: G \rightarrow G^{2}{ }^{2}$ The relations obeyed by $\eta$ and $\varepsilon$
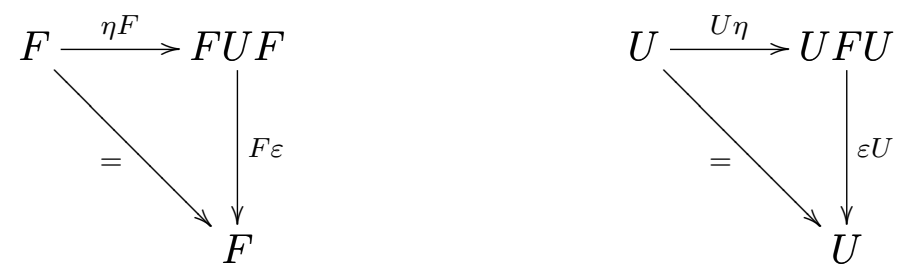

imply that $\mathbf{G}=(G, \varepsilon, \delta)$ is a cotriple in $\mathbf{C}$. This fact was first recognized by Huber [Huber (1961)].

${ }^{a}$ Editor's footnote: The $\mathbf{C}$ here and in the sentence before were $\mathbf{B}$ in the original, but as this was a mistake they are corrected now. 
1.1 Additive example: Homology of modules. Let $R$-Mod be the category of (left) $R$-modules. Let $\mathbf{G}=(G, \varepsilon, \delta)$ be the cotriple generated by the adjoint pair

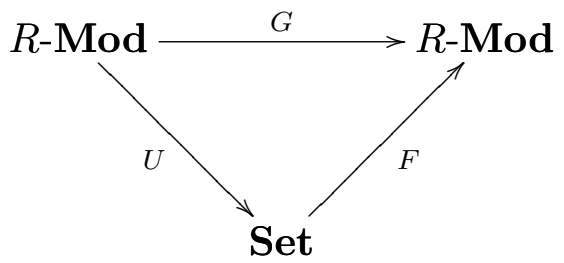

$U$ is the usual underlying set functor, $F \dashv U$ is the free $R$-module functor. Thus we have $M G=R(M)$, the free $R$-module with the elements of $M$ as basis, and the counit $M \varepsilon: M g \rightarrow M$ is the map which takes each basis element into the same element in $M$ (just the usual way of starting to construct an $R$-free resolution of $M)$. The comultiplication $M \delta: M G \rightarrow M G^{2}$ we leave to the reader.

Later we shall show that the complex

$$
0 \longleftarrow M \longleftarrow \partial_{0} \longleftarrow \partial_{1} \stackrel{\partial_{1}}{\longleftarrow} M G^{2} \longleftarrow \cdots \stackrel{\partial_{n}}{\longleftarrow} M G^{n+1} \longleftarrow \cdots
$$

where $\partial_{n}=\sum(-1)^{i} M \varepsilon_{i}$ for $0 \leq i \leq n$, is an $R$-free resolution of $M$ (the only issue is exactness). Taking as coefficient functors $M E=A \otimes_{R} M$ or $M E=\operatorname{Hom}_{R}(M, A)$, we obtain $H_{n}\left(M, A \otimes_{R}\right)$ and $H^{n}\left(M, \operatorname{Hom}_{R}(, A)\right)$ as $n$-th homology or cohomology of

$$
\begin{gathered}
0 \longleftarrow A \otimes_{R} M G \longleftarrow A \otimes_{R} M G^{2} \leftarrow \cdots \\
0 \longrightarrow \operatorname{Hom}_{R}(M G, A) \longrightarrow \operatorname{Hom}_{R}\left(M G^{2}, A\right) \longrightarrow \cdots
\end{gathered}
$$

That is, $H_{n}=\operatorname{Tor}_{n}^{R}(A, M)$ and $H^{n}=\operatorname{Ext}_{R}^{n}(M, A)$.

Since $R$-modules are an additive category and the coefficient functors considered were additive, we could form the alternating sum of the face operators to obtain a chain complex in $R$-Mod before applying the coefficient functor.

As another example of this we mention the Eckmann-Hilton homotopy groups $\Pi_{n}(M, N)$ (as re-indexed in accordance with [Huber (1961)]). These are the homology groups of the complex

$$
0 \longleftarrow \operatorname{Hom}_{R}(M, N G) \longleftarrow \operatorname{Hom}_{R}\left(M, N G^{2}\right) \longleftarrow \cdots
$$

Of course, in these examples the homology should have a subscript $\mathbf{G}$ to indicate that the cotriple relative to the underlying category of sets was used to construct the resolution. Other underlying categories and cotriples are possible. For example, if

$$
K \stackrel{\varphi}{\longrightarrow} R
$$

is a ring map, we get an adjoint pair

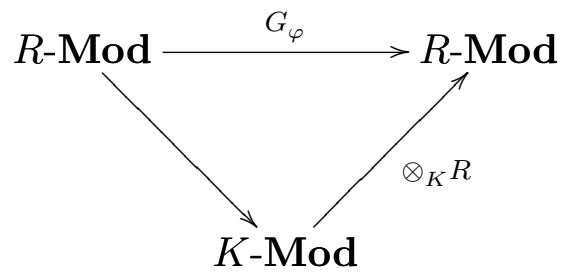


where the underlying [functor] is restriction of operators to $K$ by means of $\varphi$. We have $M G_{\varphi}=M \otimes_{K} R$. The standard resolution is

$$
M \longleftarrow M \otimes_{K} R \leftleftarrows M \otimes_{K} R \otimes_{K} R \leftleftarrows
$$

Using the above coefficient functors we will find that the homology and cohomology are Hochschild's $K$-relative Tor and Ext [Hochschild (1956)]:

$$
\begin{aligned}
H_{n}\left(M, A \otimes_{R}\right) & =\operatorname{Tor}_{n}^{\varphi}(A, M) \\
H^{n}\left(M, \operatorname{Hom}_{R}(, A)\right) & =\operatorname{Ext}_{\varphi}^{n}(M, A)
\end{aligned}
$$

Hochschild actually considered a subring $K \rightarrow R$ and wrote $\operatorname{Tor}^{(R, K)}$, etc.

We now turn to homology of groups and algebras. A useful device in the non-additive generalizations of homology theory is the comma category $(\mathbf{C}, X)$ of all objects (of a given category $\mathbf{C}$ ) over a fixed object $X$. That is, an object of $(\mathbf{C}, X)$ is a map $C \rightarrow X$, and a map of $(\mathbf{C}, X)$ is a commutative triangle

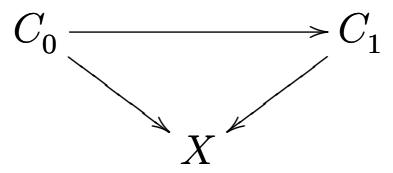

A cotriple $\mathbf{G}=(G, \varepsilon, \delta)$ in $\mathbf{C}$ naturally operates in $(\mathbf{C}, X)$ as well. The resulting cotriple $(\mathbf{G}, X)$ has

$$
\begin{gathered}
(C \stackrel{p}{\longrightarrow} X)(G, X)=C G \stackrel{C \varepsilon}{\longrightarrow} C \stackrel{p}{\longrightarrow} X \\
(C \longrightarrow X)(\varepsilon, X)=C G \stackrel{C \varepsilon}{\longrightarrow} C \\
(C \longrightarrow X)(\delta, X)=C G \stackrel{C \delta}{\longrightarrow} C G^{2}
\end{gathered}
$$

The standard $(\mathbf{G}, X)$-resolution of an object $C \rightarrow X$ over $X$ comes out in the form

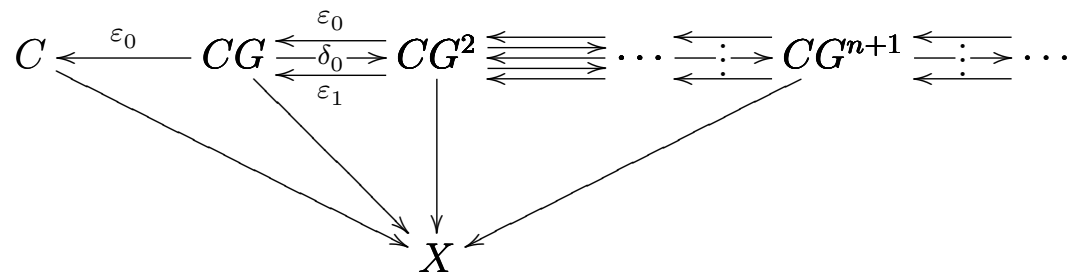

In other words, the usual faces and degeneracies turn out to be maps over X.

Homology groups $H_{n}(C, E)_{(\mathbf{G}, X)}$ are then defined, when $E:(\mathbf{C}, X) \rightarrow \mathscr{A}$ is a coefficient functor. We could write, with greater precision, $H_{n}(p, E)_{(\mathbf{G}, X)}$, or with less, $H_{n}(C, E)_{X}$ or $H_{n}(C, E)$, leaving $X$ understood. 
Usually the coefficient functors involve a module over the terminal object $X$. This can be treated as a module over all the objects of $(\mathbf{C}, X)$ simultaneously, by pullback via the structural maps to $X$. For example, derivations or differentials with values in an $X$-module become functors on the category of all algebras over $X$. This is the way in which homology and cohomology of algebras arise.

1.2 Homology of Groups. Let $\mathbf{G r}$ be the category of groups and $\mathbf{G}$ the cotriple arising from

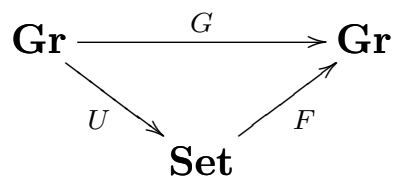

Thus $\Pi G$ is the free group on the underlying set of $\Pi$, and the counit $\Pi G \rightarrow \Pi$ is the natural surjection of the free group onto $\Pi$.

If $W \rightarrow \Pi$ is a group over $\Pi$ and $M$ is a left $\Pi$-module, a derivation $f: W \rightarrow M$ (over $\Pi$ ) is

a function such that $\left(w w^{\prime}\right) f=w \cdot w^{\prime} f+w f(W \rightarrow \Pi$ allows $W$ to act on $M)$. The abelian group of such derivations, $\operatorname{Der}(W, M)_{\Pi}$, gives a functor $(\mathbf{G r}, \Pi)^{\mathrm{op}} \rightarrow \mathbf{A b}$. We define the cohomology of $W \rightarrow \Pi$ with coefficients in $M, H^{n}(W, M)_{\Pi}$ (relative to $\mathbf{G}$ ) as the cohomology of the cochain complex

$$
0 \longrightarrow \operatorname{Der}(W G, M)_{\Pi} \longrightarrow \operatorname{Der}\left(W G^{2}, M\right)_{\Pi} \longrightarrow \cdots \longrightarrow \operatorname{Der}\left(W G^{n+1}, M\right)_{\Pi} \longrightarrow \cdots
$$

It is known that this theory coincides with Eilenberg-Mac Lane cohomology except for a shift in dimension [Barr \& Beck (1966)]

$$
H^{n}(W, M)_{\Pi} \stackrel{\cong}{\longrightarrow} \begin{cases}\operatorname{Der}(W, M)_{\Pi} & \text { for } n=0 \\ H_{E-M}^{n+1}(W, M) & \text { for } n>0\end{cases}
$$

Derivations $W \rightarrow M$ are represented by a $\Pi$-module of differentials of $W$ (over $\Pi$ ) which we write as $\operatorname{Diff}_{\Pi}(W)$ :

$$
\begin{aligned}
\operatorname{Der}(W, M) \Pi & \cong \operatorname{Hom}_{W}(I W, M) \\
& =\operatorname{Hom}_{\Pi}\left(\mathbf{Z} \Pi \otimes_{W} I W, M\right)
\end{aligned}
$$

Hence $\operatorname{Diff}_{\Pi}(W)=\mathbf{Z} \Pi \otimes_{W} I W$. (It is well known that the augmentation ideal $I W=$ $\operatorname{ker}(\mathbf{Z} W \rightarrow \mathbf{Z})$ represents derivations of $W$ into $W$-modules [Cartan \& Eilenberg (1956), Mac Lane (1963)]. This is fudged by $\mathbf{Z} \Pi \otimes_{W}$ to represent derivations into $\Pi$-modules.)

The homology of $W \rightarrow \Pi$ with coefficients in a right $\Pi$-module $M$ is defined as the homology of

$0 \leftarrow M \otimes_{\mathbf{z} \Pi} \operatorname{Diff}_{\Pi}(W G) \leftarrow M \otimes_{\mathbf{z} \Pi} \operatorname{Diff}_{\Pi}\left(W G^{2}\right) \leftarrow \cdots \leftarrow M \otimes_{\mathbf{z} \Pi} \operatorname{Diff}_{\Pi}\left(W G^{n+1}\right) \leftarrow \cdots$

Then

$$
H_{n}(W, M)_{\Pi} \stackrel{\cong}{\longrightarrow} \begin{cases}M \otimes_{\mathbf{z \Pi}} \operatorname{Diff}_{\Pi}(W)=M \otimes_{\mathbf{z} \Pi} I W & \text { for } n=0 \\ H_{n+1}^{E-M}(W, M) & \text { for } n>0\end{cases}
$$


This is because $\left(\operatorname{Diff}_{\Pi}\left(W G^{n+1}\right)\right)_{n \geq-1}$ is a $\Pi$-free resolution of $\operatorname{Diff}_{\Pi}(W)$, and as [Cartan \& Eilenberg (1956), Mac Lane (1963)] show, the Eilenberg-Mac Lane homology can be identified with $\operatorname{Tor}_{n+1}^{\mathbf{Z W}}(\mathbf{Z}, N)=\operatorname{Tor}_{n}^{\mathbf{Z W}}(I W, N)$. П-Freeness is because $\operatorname{Diff}_{\Pi}(W G)=$ $\mathbf{Z} \Pi \otimes_{W} I(W G)$, and $I(W G)$ is well known to be $W G$-free. As for acyclicity, the cohomology of

$$
0 \longrightarrow \operatorname{Hom}_{\Pi}\left(\operatorname{Diff}_{\Pi}(W), Q\right) \longrightarrow \operatorname{Hom}_{\Pi}\left(\operatorname{Diff}_{\Pi}(W G), Q\right) \longrightarrow \cdots
$$

is zero in all dimensions $\geq-1$, if $Q$ is an injective $\Pi$-module; this is true because the cohomology agrees with the Eilenberg-Mac Lane theory, which vanishes on injective coefficient modules. A direct acyclic-models proof of the coincidence of the homology theories can also be given.

As special cases note: if $\Pi$ is regarded as a group over $\Pi$ by means of the identity map $\Pi \rightarrow \Pi$, the $H^{n}(\Pi, M)_{\Pi}$ and $H_{n}(\Pi, M)_{\Pi}$ are the ordinary (co-)homology groups of $\Pi$ with coefficients in a $\Pi$-module. On the other hand, if $\Pi=1$, any $W$ can be considered as a group over $\Pi$. Since a 1-module is just an abelian group, $\operatorname{Diff}_{1}(W)=W /[W, W]$, [which is] $W$ abelianized, i.e. with its commutator subgroup divided out. The (co-)homology is that of $W$ with coefficients in a trivial module.

REMARK. [Beck (1967), Barr \& Beck (1966)] Via interpretation as split extensions, $\Pi$-modules can be identified with the abelian group objects in the category $(\mathbf{G r}, \Pi)$. $\operatorname{Der}(W, M)_{\Pi}$ is then the abelian group of maps in $(\mathbf{G r}, \Pi)$ :

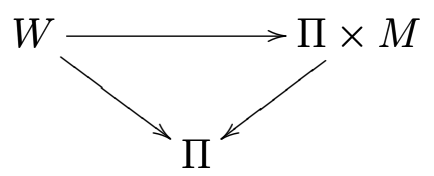

$\operatorname{Diff}_{\Pi}$ is just the free abelian group functor, that is, the left adjoint of the forgetful functor

$$
(\mathbf{G r}, \Pi) \leftarrow \mathbf{A b}(\mathbf{G r}, \Pi)=\Pi-\mathbf{M o d}
$$

where $\mathbf{A b}(\mathbf{G r}, \Pi)$ denotes the abelian groups in $(\mathbf{G r}, \Pi)$.

For general triple cohomology this interpretation is essential. In particular, the analogue of Diff exists for any category tripleable over Set, provided the triple has a rank in the sense of [Linton (1966a)].

For the next example we need the comma category $(X, \mathbf{C})$ of objects and maps in $\mathbf{C}$ under $X$. An object of this category is a map $X \rightarrow Y$, a map is a commutative triangle

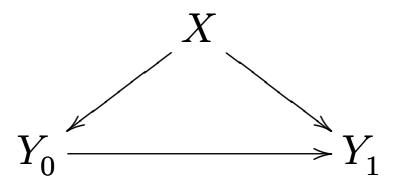

Assuming $\mathbf{C}$ has coproducts $X * Y$, a cotriple $\mathbf{G}=(G, \varepsilon, \delta)$ in $\mathbf{C}$ naturally induces a 
cotriple $(X, \mathbf{G})=((X, G), \ldots)$ in $(X, \mathbf{C})$ :

$$
\begin{aligned}
& (X \stackrel{f}{\longrightarrow} Y)(X, G)=X \longrightarrow X * Y G \quad \text { (coproduct injection) }
\end{aligned}
$$

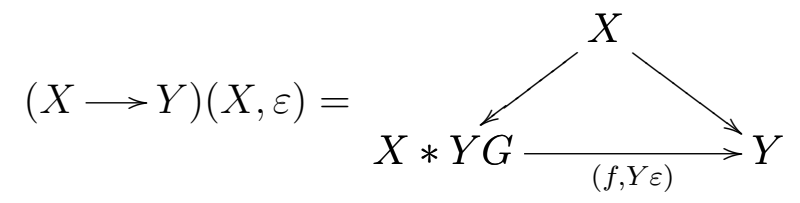

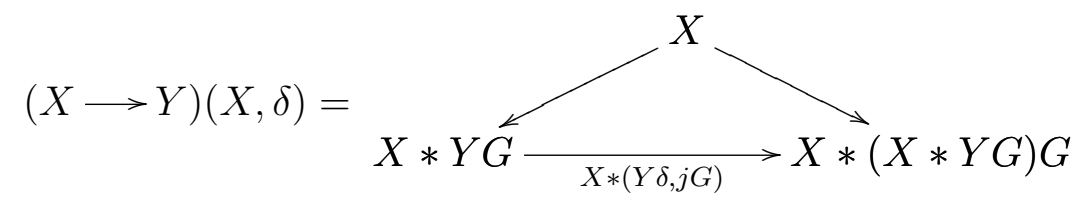

where $j: Y G \rightarrow X * Y G$ is a coproduct injection.

Actually, the coproduct $X *()$ defines an adjoint pair of functors $(X, \mathbf{C}) \rightarrow \mathbf{C} \rightarrow(X, \mathbf{C})$; the right adjoint is $(X \rightarrow C) \mapsto C$, the left adjoint is $C \mapsto(X \rightarrow X * C)$. By a general argument [Huber (1961)], the composition

$$
(X, \mathbf{C}) \longrightarrow \mathbf{C} \stackrel{G}{\longrightarrow} \mathbf{C} \stackrel{X *()}{\longrightarrow}(X, \mathbf{C})
$$

is then a cotriple in $(X, \mathbf{C})$, namely $(X, \mathbf{G})$.

Replacing $(X, \mathbf{C}) \rightarrow \mathbf{C} \rightarrow(X, \mathbf{C})$ by an arbitrary adjoint pair and specializing $\mathbf{G}$ to the identity cotriple proves the remark preceding (1.1).

Homology and cohomology relative to the cotriple $(X, \mathbf{G})$ will be studied in more detail in Section 8. This cotriple enters in a rather mild way into:

1.3 Homology of commutative Rings and algebras. Let Comm be the category of commutative rings. For $A \in \mathbf{C o m m}$ let $(A, \mathbf{C o m m})$ be the category of commutative rings under $A$, that is, maps $A \rightarrow B \in \mathbf{C o m m}$. Thus ( $A$, Comm) is our notation for the category of commutative $A$-algebras. We review the notions of differentials and derivations in this category.

For the same reason as in the category of groups we place ourselves in a category of algebras over a fixed commutative ring $D$, that is, in a double comma category $(A, \mathbf{C o m m}, D)$; here an object is an $A$-algebra $A \rightarrow B$ equipped with a map $B \rightarrow D$, and a map is a commutative diagram

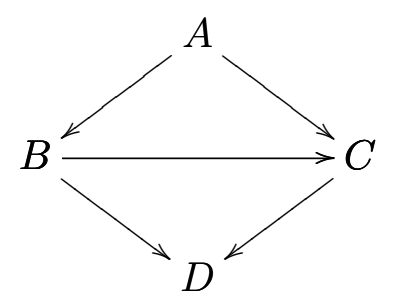

If $M$ is a $D$-module, an $A$-derivation $B \rightarrow M$ is an $A$-linear function satisfying $\left(b b^{\prime}\right) f=b^{\prime} \cdot b f+b \cdot b^{\prime} f$, where $B \in(A, \mathbf{C o m m}, D)$ and $A$ and $B$ act on $M$ via the given 
maps $A \rightarrow B \rightarrow D$. Such modules of derivations define a functor

$$
(A, \text { Comm }, D)^{\text {op }} \stackrel{A-\operatorname{Der}(, M)_{D}}{\longrightarrow} D \text {-Mod }
$$

This eventually gives rise to cohomology.

As is well known, any $A$-derivation $B \rightarrow M$, where $M$ is a $B$-module, factors uniquely through a $B$-module map

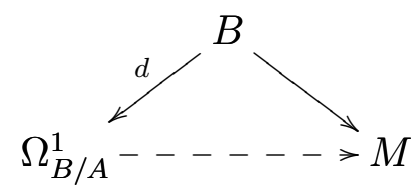

where $\Omega_{B / A}^{1}$ is the $B$-module of $A$-differentials of $B$, and $d$ is the universal such derivation. $\Omega_{B / A}^{1}$ can be viewed as $I / I^{2}$ where $I=\operatorname{ker}\left(B \otimes_{A} B \rightarrow B\right)$ and $d b=b \otimes 1-1 \otimes b$, or as the free $B$-module on symbols $d b$ modulo $d\left(b+b^{\prime}\right)=d b+d b^{\prime}$ as well as $d(a b)=a \cdot d b$ and $d\left(b b^{\prime}\right)=b^{\prime} \cdot d b+b \cdot d b^{\prime}$ [Lichtenbaum \& Schlessinger (1967), Grothendieck \& Dieudonné (1964)]. [A] universal [object] for $A$-derivations of $B \rightarrow M$, where $M$ is a $D$-module, is then

$$
\operatorname{Diff}_{D}(A \rightarrow B)=\Omega_{B / A}^{1} \otimes_{B} D
$$

The functor which is usually used as coefficients for homology is

$$
(A, \mathbf{C o m m}, D) \stackrel{\left.\left.\operatorname{Diff}_{D}(A \rightarrow()) \otimes_{D} M=\Omega_{(}^{1}\right) / A^{\otimes_{(}}\right)^{M}}{\longrightarrow} D \text {-Mod }
$$

There are two natural ways of defining homology in the category of $A$-algebras (over $D$ ), depending on the choice of cotriple, or equivalently, choice of the underlying category.

First let $\mathbf{G}=(G, \varepsilon, \delta)$ be the cotriple in the category of commutative rings arising from the adjoint pair

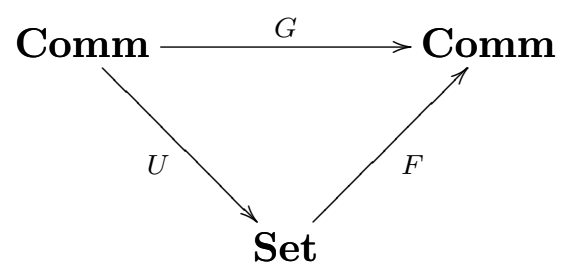

Then $C G=\mathbf{Z}[C]$, the polynomial ring with the elements of $C$ as variables; the counit $C G \rightarrow C$ is the map defined by sending the variable $c$ [to the element] $c \in C$. This cotriple operates in (Comm, $D)$ in the natural fashion described before (1.2).

Now consider the category $(A, \mathbf{C o m m})$ of commutative $A$-algebras. According to the remarks preceding this section, $\mathbf{G}$ gives rise to a cotriple $(A, \mathbf{G})$ in this category. Since the coproduct in the category $\mathbf{C o m m}$ is $A \otimes_{\mathbf{z}} B$, we have

$$
\begin{aligned}
(A \rightarrow C)(A, G) & =A \rightarrow A \otimes_{\mathbf{Z}} C G \\
& =A \rightarrow A \otimes_{\mathbf{Z}} \mathbf{Z}[C] \\
& =A \rightarrow A[C]
\end{aligned}
$$


the polynomial $A$-algebra with the elements of $C$ as variables. This cotriple is just that which is induced by the underlying set and free $A$-algebra functors

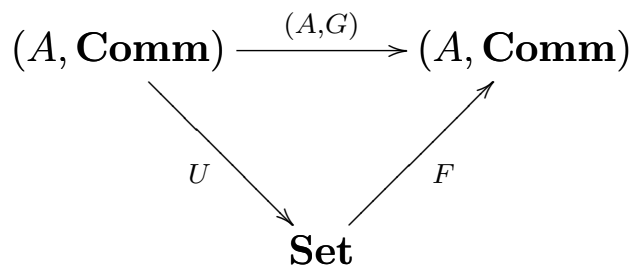

Furthermore, $(A, \mathbf{G})$ operates in the category of $A$-algebras over $D,(A, \mathbf{C o m m}, D)$, the values of $(A, \mathbf{G}, D)$ being given by:

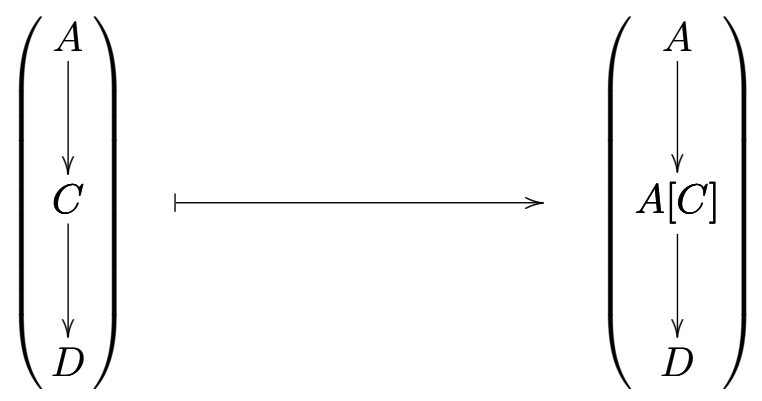

The counit is:

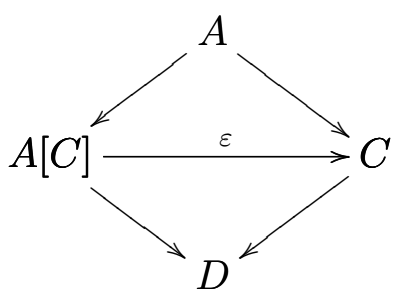

If $M$ is a $D$-module we thus have homology and cohomology $D$-modules $H_{n}(C, M)$ and $H^{n}(C, M)$ for $n \geq 0$, writing simply $C$ for an $A$-algebra over $D$. These are defined by

$$
\begin{aligned}
H_{n}(C, M) & =H_{n}\left[\left(\operatorname{Diff}_{D}\left(C(A, G)^{p+1}\right) \otimes_{D} M\right)_{p \geq 0}\right] \\
& =H_{n}\left[\left(\Omega_{A[\cdots[C] \cdots] / A}^{1} \otimes_{A} M\right)_{p \geq 0}\right]
\end{aligned}
$$

where there are $p+1$ applications of the $A$-polynomial operation to $C$ in dimension $p$, and by

$$
\begin{aligned}
H^{n}(C, M) & =H^{n}\left[\left(A-\operatorname{Der}_{D}\left(C(A, G)^{p+1}, M\right)\right)_{p \geq 0}\right] \\
& =H^{n}\left[\left(A-\operatorname{Der}_{D}(A[\cdots[C] \cdots], M)\right)_{p \geq 0}\right]
\end{aligned}
$$

again with $p+1 A[$ ]'s.

This homology theory of commutative algebras over $D$ coincides with those considered in [André (1967), Quillen (1967)]; of course, one generally simplifies the setting slightly 
by taking $C=D$ above. Both of these papers contain proofs that the cotriple theory coincides with theirs. The homology theory of [Lichtenbaum \& Schlessinger (1967)] also agrees.

This theory, however it is described, is called the "absolute" homology theory of commutative algebras. The term arises as a reference to the underlying category which is involved, namely that of sets; no underlying object functor could forget more structure. But it also seems germane to consider so-called relative homology theories of algebras for which the underlying category is something else, usually a category of modules.

As an example of this, consider the homology theory in $(A, \mathbf{C o m m})$ comming from the adjoint functors

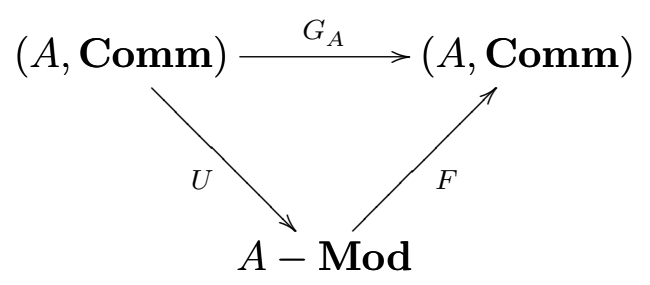

That is

$$
(A \rightarrow C) G_{A}=A+C+\frac{C \otimes_{A} C}{S_{2}}+\frac{C \otimes_{A} C \otimes_{A} C}{S_{3}}+\cdots
$$

the symmetric $A$-algebra on $C$ (the $S$ 's are the symmetric groups). Note that this cotriple is not of the form $(A, \mathbf{G})$ for any cotriple $\mathbf{G}$ on the category of commutative rings. Exactly as above we now have homology and cohomology groups

$$
\begin{aligned}
H_{n}(C, M) & =H_{n}\left[\left(\operatorname{Diff}_{D}\left(C G_{A}^{p+1}\right) \otimes_{D} M\right)_{p \geq 0}\right] \\
& =H_{n}\left[\left(\Omega_{C G_{A}^{1} / A}^{1} \otimes_{D} M\right)_{p \geq 0}\right] \\
H^{n}(C, M) & =H^{n}\left[\left(A-\operatorname{Der}\left(C G_{A}^{p+1}, M\right)_{D}\right)_{p \geq 0}\right]
\end{aligned}
$$

where $M$ is a $D$-module, and we are writing $C$ instead of $A \rightarrow C$ for an $A$-algebra.

These two cohomology theories should really be distinguished by indicating the cotriple used to define them:

$$
\begin{aligned}
H^{n}(C, M)_{(A, \mathbf{G})} & =\text { absolute theory, relative to sets } \\
H^{n}(C, M)_{\mathbf{G}_{A}} & =\text { theory relative to } A \text {-modules }
\end{aligned}
$$

The following is an indication of the difference between them: if $C=D$ and $M$ is a $C$-module, then $H^{1}(C, M)_{(A, \mathbf{G})}$ classifies commutative $A$-algebra extensions $E \rightarrow C$ such that $I=\operatorname{ker}(E \rightarrow C)$ is an ideal of $E$ with $I^{2}=0$, and such that there exists a lifting of the counit

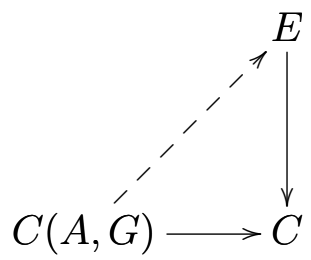


$H^{1}(C, M)_{\mathbf{G}_{A}}$ classifies those extensions with kernel of square zero that have liftings

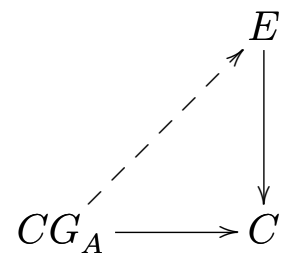

The absolute lifting condition is equivalent to the existence of a set section of $E \rightarrow C$, i.e. to surjectivity, the $A$-relative condition to the existence of an $A$-linear splitting of $E \rightarrow C$, as one can easily check. The relative theory is thus insensitive to purely $A$-linear phenomena, while the absolute theory takes all the structure into account. (We refer to [Beck (1967)] for details on classification of extensions).

The $A$-relative cohomology theory has been studied but little. Harrison has given an $A$-relative theory in [Harrison (1962)] ( $A$ was a ground field but his formulas are meaningful for any commutative ring). Barr [Barr (1968)] has proved that

$$
H^{n}(C, M) \cong \begin{cases}\operatorname{Der}(C, M) & \text { for } n=0 \\ \operatorname{Harr}^{n+1}(C, M) & \text { for } n>0\end{cases}
$$

if $A$ is a field of characteristic zero.

1.4 Homology of Associative $K$-Algebras. Let $\mathbf{G}_{K}$ be the cotriple relative to the underlying category of $K$-modules:

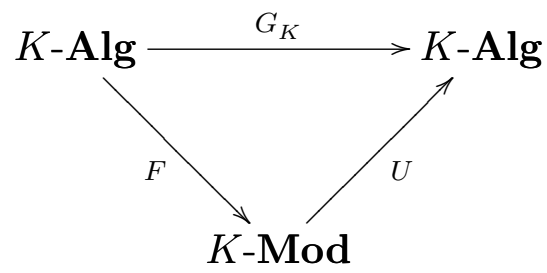

Thus if $\Lambda$ is an associative algebra with unit over the commutative ring $K$, then

$$
\Lambda G_{K}=K+\Lambda+\Lambda \otimes \Lambda+\cdots
$$

the $K$-tensor algebra.

If $\Gamma \rightarrow \Lambda$ and $M$ is a $\Lambda$ - $\Lambda$-bimodule, we define $H^{n}(\Gamma, M)_{\Lambda}$ as the cohomology of the cosimplicial object

$$
0 \longrightarrow \operatorname{Der}\left(\Gamma G_{K}, M\right)_{\Lambda} \Longrightarrow \operatorname{Der}\left(\Gamma G_{K}^{2}, M\right)_{\Lambda} \rightleftarrows \cdots \longrightarrow \operatorname{Der}\left(\Gamma G_{K}^{n+1}, M\right)_{\Lambda} \longrightarrow \cdots
$$

It is known that this coincides with Hochschild cohomology [Barr (1966), Barr \& Beck (1966)]:

$$
H^{n}(\Gamma, M)_{\Lambda} \cong \begin{cases}\operatorname{Der}(\Gamma, M) & \text { for } n=0 \\ \operatorname{Hoch}^{n+1}(\Gamma, M) & \text { for } n>0\end{cases}
$$


The universal object for $K$-linear derivations $\Gamma \rightarrow M$, where $M$ is a two-sided $\Lambda$ module, is

$$
\operatorname{Diff}_{\Lambda}(\Gamma)=\operatorname{Diff}_{\Gamma}(\Gamma) \otimes_{\Gamma^{e}} \Lambda^{e}=\Lambda \otimes_{\Gamma} J \Gamma \otimes_{\Gamma} \Lambda
$$

where $J \Gamma$ is the kernel of the multiplication $\Gamma^{e}=\Gamma \otimes_{K} \Gamma^{\mathrm{op}} \rightarrow \Gamma$ and represents derivations of $\Gamma$ into $\Gamma$-modules [Cartan \& Eilenberg (1956), Mac Lane (1963)]. The homology of $\Gamma \rightarrow \Lambda$ with coefficients in $M$ is defined as the homology of the complex

$$
0 \longleftarrow \operatorname{Diff}_{\Lambda}\left(\Gamma G_{K}\right) \otimes_{\Lambda^{e}} M \longleftarrow \partial_{1} \operatorname{Diff}_{\Lambda}\left(\Gamma G_{K}^{2}\right) \otimes_{\Lambda^{e}} M \longleftarrow \stackrel{\partial_{2}}{\longleftarrow} \cdots
$$

[Barr (1966)] proves that $\left(\operatorname{Diff}_{\Lambda}\left(\Gamma G_{K}^{n+1}\right)\right)_{n \geq-1}$ is a $K$-contractible complex of $\Lambda^{e}$-modules which are free relative to the underlying category of $K$-modules. Thus

$$
H_{n}(\Gamma, M) \stackrel{\cong}{\longrightarrow} \begin{cases}\operatorname{Diff}_{\Lambda}(\Gamma) \otimes_{\Lambda^{e}} M & \text { for } n=0 \\ \operatorname{Hoch}_{n+1}(\Gamma, M) & \text { for } n>0\end{cases}
$$

the last being Hochschild homology as defined in [Mac Lane (1963), Chapter X].

The foregoing is a $K$-relative homology theory for associative $K$-algebras, in the sense of (1.3). There is also an absolute theory, due to Shukla [Shukla (1961)], which Barr has proved coincides with the cotriple theory relative to the category of sets (with the usual dimension shift) [Barr (1967)]. We shall not deal with this absolute theory in this paper.

This concludes the present selection of examples. A further flock of examples will appear in Section 10.

\section{Properties of the $H_{n}(X, E)_{\mathbf{G}}$ as functors of $X$, including exact sequences}

Objects of the form $X G$, that is, values of the cotriple $\mathbf{G}$, can be thought of as free relative to the cotriple. Free objects are acyclic:

Proposition (2.1).

$$
\begin{gathered}
H_{0}(X G, E)_{\mathbf{G}} \stackrel{\lambda}{\cong} X G E \\
H_{n}(X G, E)_{\mathbf{G}}=0 \quad \text { for } n>0
\end{gathered}
$$

An object $P$ is called $\mathbf{G}$-projective if $P$ is a retract of some value of $G$, or equivalently, if there is a map $s: P \rightarrow P G$ such that $s \circ P \varepsilon=P$. G-projectives obviously have the same acyclicity property.

To prove (2.1), we just recall from the Introduction that there is a contraction in the simplicial object $\left(X G G^{n+1} E\right)_{n \geq-1}$.

If $f: X \rightarrow Y$ in $\mathbf{C}$, we define "relative groups" or homology groups of the map, $H_{n}(f, E)_{\mathbf{G}}$ for $n \geq 0$, such that the following holds: 
Proposition (2.2). If $X \rightarrow Y$ in $\mathbf{C}$, there is an exact sequence

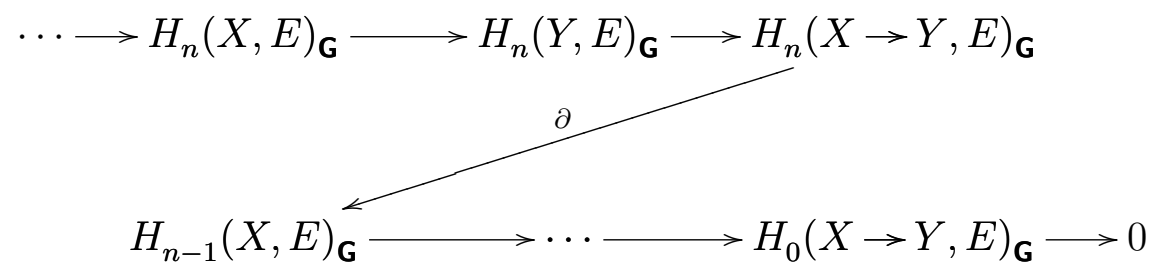

Proposition (2.3). If $X \rightarrow Y \rightarrow Z$ in $\mathbf{C}$, there is an exact sequence

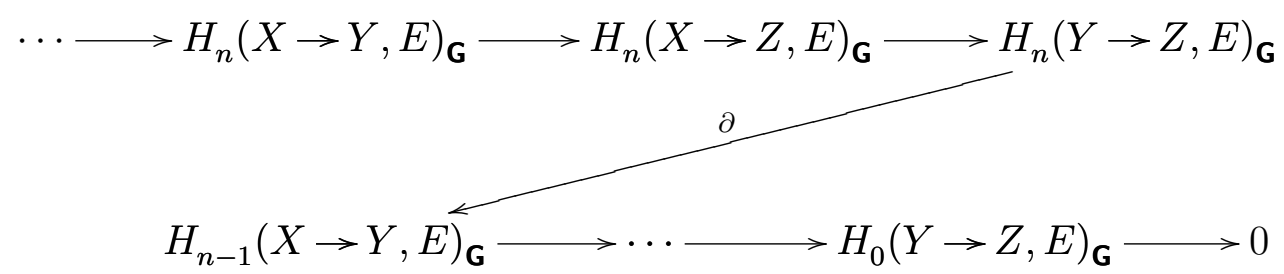

If 0 is an initial object in $\mathbf{C}$, that is, if there is a unique map $0 \rightarrow X$ for every $X$, then 0 is $\mathbf{G}$-projective and

$$
\begin{aligned}
& H_{0}(X, E) \stackrel{\cong}{\longrightarrow} H_{0}(0 \rightarrow X, E) \\
& H_{n}(X, E) \stackrel{\cong}{\longrightarrow} H_{n}(X G \stackrel{\varepsilon}{\longrightarrow} X, E) \quad \text { for } n>0
\end{aligned}
$$

Examples of these sequences will be deferred to Section 8 . There we will show that under certain conditions the homology group $H_{n}(X \rightarrow Y, E)$ can be interpreted as a cotriple homology group relative to the natural cotriple in the category $(X, C)$. For one thing, it will turn out that the homology of a map of commutative rings, $H_{n}(A \rightarrow B)$, is just the homology of $B$ as an $A$-algebra.

Imitative though these sequences may be of theorems in algebraic topology, we don't know how to state a uniqueness theorem for G-homology in our present context.

As to the definition of the relative groups, we just let

$$
H_{n}(X \stackrel{f}{\longrightarrow} Y, E)_{\mathbf{G}}=H_{n}(C f)
$$

where $C f$ is the mapping cone of the chain transformation

$$
f G^{n+1} E: X G^{n+1} E \rightarrow Y G^{n+1} E \quad \text { for } n \geq 0
$$

That is,

$$
\begin{aligned}
& (C F)_{n}= \begin{cases}Y G^{n+1} E \oplus X G^{n} E & \text { for } n>0 \\
Y G E & \text { for } n=0\end{cases} \\
& \partial_{n}=\left(\begin{array}{cc}
\partial_{Y} & 0 \\
f G^{n+1} E & -\partial_{X}
\end{array}\right):(C f)_{n} \rightarrow(C f)_{n-1} \quad \text { for } n \geq 2 \\
& \partial_{1}=\left(\begin{array}{c}
\partial_{Y} \\
f G E
\end{array}\right):(C f)_{1} \rightarrow(C f)_{0}
\end{aligned}
$$


(These matrices act on row vectors from the right, $\partial_{X}$ and $\partial_{Y}$ indicate boundary operators in the standard complexes of $X$ and $Y$.)

(2.2) follows from the exact sequence of chain complexes

$$
0 \longrightarrow\left(Y G^{n+1} E\right)_{n \geq 0} \longrightarrow C f \stackrel{\Pi}{\longrightarrow}\left(X G^{n+1} E\right)_{n \geq 0} \longrightarrow 0
$$

where the projection $\Pi$ is a chain transformation of degree -1 .

(2.3) follows from (2.2) by routine algebraic manipulation ([Eilenberg \& Steenrod (1952), Wall (1966)]).

\section{Axioms for the $H_{n}(X, E)_{\mathbf{G}}$ as functors of the abelian variable $E$}

In this section we show that the functors $H_{n}(, E)_{\mathbf{G}}: \mathbf{C} \longrightarrow \mathscr{A}$ are characterized by the following two properties. (In Section 4 it will appear that they are characterized by a little bit less.)

\subsection{G-ACYCLICITY.}

$$
\begin{aligned}
& H_{0}(, G E)_{\mathbf{G}} \stackrel{\cong}{\lambda} G E, \\
& H_{n}(, G E)_{\mathbf{G}}=0, \quad n>0 .
\end{aligned}
$$

3.2. G-Connectedness. If $0 \longrightarrow E^{\prime} \longrightarrow E \longrightarrow E^{\prime \prime} \longrightarrow 0$ is a $\mathbf{G}$-short exact sequence of functors $\mathbf{C} \longrightarrow \mathscr{A}$, then there is a long exact sequence in homology:

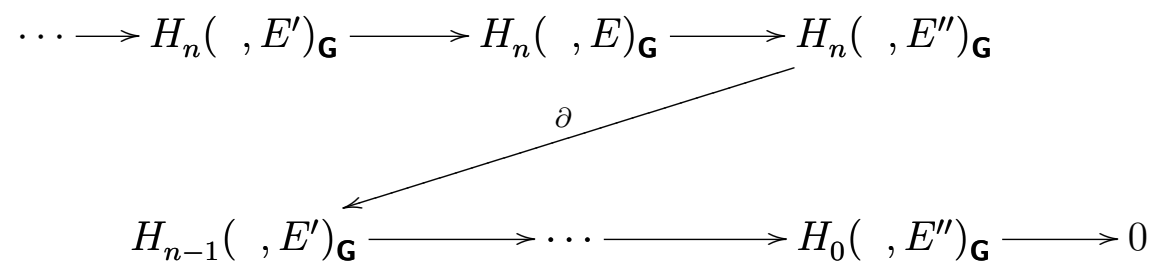

The acyclicity is trivial: as mentioned in the Introduction, the simplicial object $X G * G E$ always has a contraction by virtue of

$$
G E \stackrel{\delta E}{\longrightarrow} G(G E) .
$$

For the homology sequence, we define a sequence of functors $0 \longrightarrow E^{\prime} \longrightarrow E \longrightarrow E^{\prime \prime} \longrightarrow 0$ to be $\mathbf{G}$-exact if it is exact in the (abelian) functor category $(\mathbf{C}, \mathscr{A})$ after being composed with $G: \mathbf{C} \longrightarrow \mathbf{C}$, i.e., if and only if $0 \longrightarrow X G E^{\prime} \longrightarrow X G E \longrightarrow X G E^{\prime \prime} \longrightarrow 0$ is an exact sequence in $\mathscr{A}$ for every object $X \in \mathbf{C}$. In this event we get a short exact sequence of chain complexes in $\mathscr{A}$,

$$
0 \longrightarrow\left(X G^{n+1} E^{\prime}\right) \longrightarrow\left(X G^{n+1} E\right) \longrightarrow\left(X G^{n+1} E^{\prime \prime}\right) \longrightarrow 0, \quad n \geq-1,
$$

from which the homology sequence is standard.

Next we show that properties 3.1 and 3.2 are characteristic of the homology theory $H_{\mathbf{G}}$. Define $\mathbf{L}=\left(L_{n}, \lambda, \partial\right)$ to be a theory of $\mathbf{G}$-left derived functors if: 
1. $\mathbf{L}$ assigns to every functor $E: \mathbf{C} \longrightarrow \mathscr{A}$ a sequence of functors $L_{n} E: \mathbf{C} \longrightarrow \mathscr{A}$, and to every natural transformation $\vartheta: E \longrightarrow E_{1}$ a sequence of natural transformations $L_{n} \vartheta: L_{n} E \longrightarrow L_{n} E_{1}, n \geq 0$, such that $L_{n}\left(\vartheta \vartheta_{1}\right)=L_{n}(\vartheta) \cdot L_{n}\left(\vartheta_{1}\right)$;

2. $\lambda$ is a natural transformation $L_{0} E \longrightarrow E$ which has property 3.1 for every functor which is of the form $G E$;

3. whenever $0 \longrightarrow E^{\prime} \longrightarrow E \longrightarrow E^{\prime \prime} \longrightarrow 0$ is a $\mathbf{G}$-exact sequence of functors $\mathbf{C} \longrightarrow \mathscr{A}$, then there is a long exact homology sequence

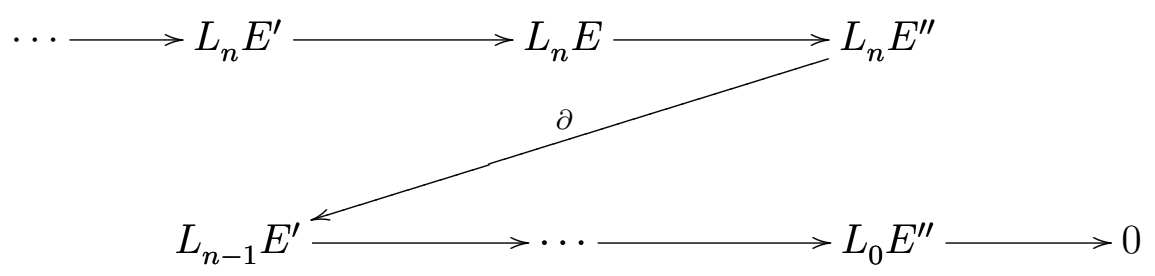

where $\partial$ actually depends on the given sequence, of course, and

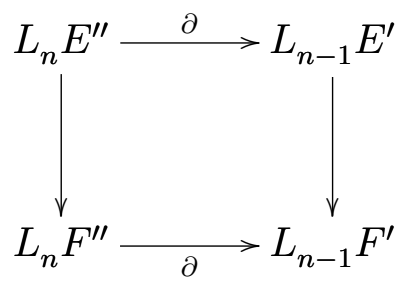

commutes for every map of $\mathbf{G}$-short exact sequences

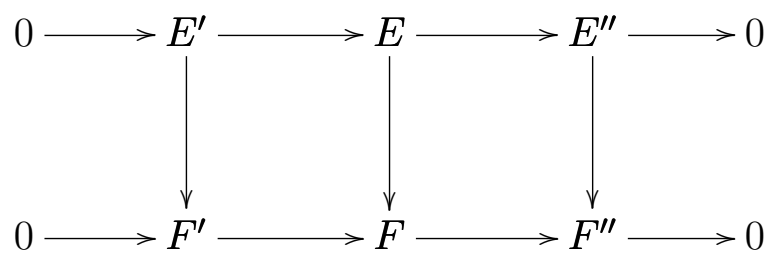

We now prove a uniqueness theorem for G-left derived functors. A proof in purely abelian-category language exists also, in fact, has existed for a long time (cf. [Röhrl (1962)] and F. Ulmer's paper in this volume.)

THEOREM (3.3). If $\mathbf{L}$ is a theory of $\mathbf{G}$-left derived functors, then there exists a unique family of natural isomorphisms

$$
L_{n} E \stackrel{\sigma_{n}}{\longrightarrow} H_{n}(, E)_{\mathbf{G}}, \quad n \geq 0,
$$

which are natural in $E$, and are compatible with the augmentations

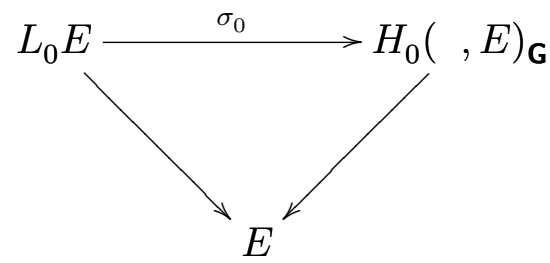


and connecting homomorphisms

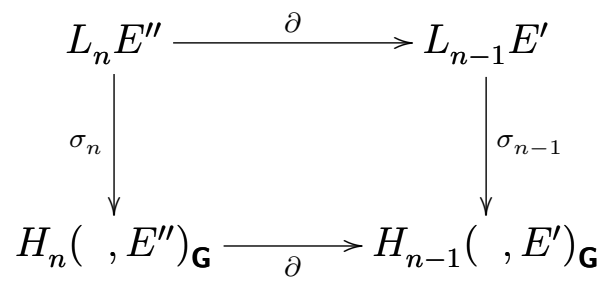

corresponding to $\mathbf{G}$-short exact sequences.

Proof. In this proof we write $H_{n}(, E)$ for $H_{n}(, E)_{\mathbf{G}}$.

As we shall prove in a moment, the following is a consequence of $\mathbf{G}$-connectedness:

Lemma (3.4). $L_{0}\left(G^{2} E\right) \stackrel{L_{0} \partial_{1}}{\longrightarrow} L_{0}(G E) \stackrel{L_{0} \partial_{0}}{\longrightarrow} L_{0} E \longrightarrow 0$ is an exact sequence in the functor category $(\mathbf{C}, \mathscr{A})$.

Supposing that $\lambda$ is a natural transformation $L_{0} E \longrightarrow E$ which is natural in $E$ as well, we get a unique map of the cokernels

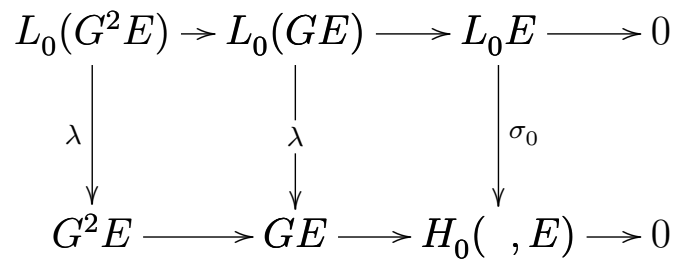

which is compatible with the augmentations:

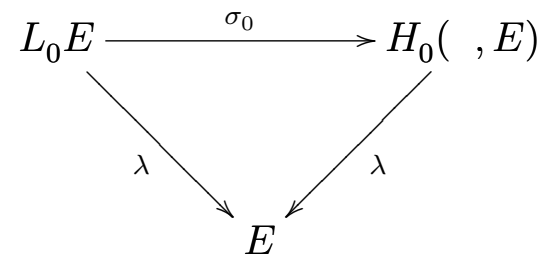

Now extend $\sigma_{0}$ inductively to a map of G-connected theories, $\sigma_{n}: L_{n} E \longrightarrow H_{n}(, E)$ for all $n \geq 0$, as follows. Let $N=\operatorname{ker}(G E \longrightarrow E)$ so that

$$
0 \longrightarrow N \stackrel{i}{\longrightarrow} G E \longrightarrow E \longrightarrow 0
$$

is an exact sequence of functors, a fortiori G-exact as well. As $\sigma_{0}$ is obviously natural in the $E$ variable, we get a diagram

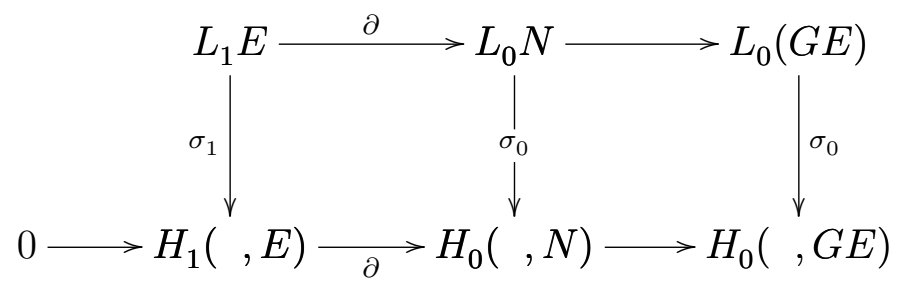


the bottom row being exact by virtue of $H_{1}(, G E)=0$. This defines $\sigma_{1}$. For $\sigma_{n}, n \geq 2$, use the diagram

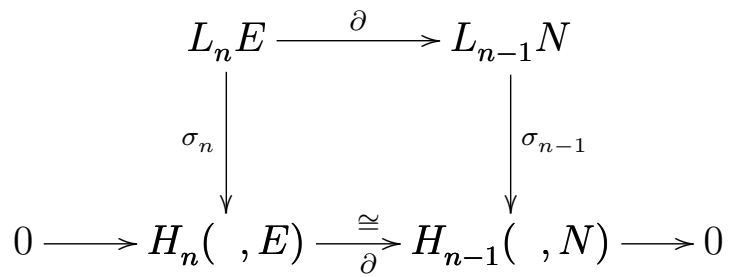

This defines all of the maps $\sigma_{n}$. But to have a map of $\mathbf{G}$-connected homology theories, we must verify that each square

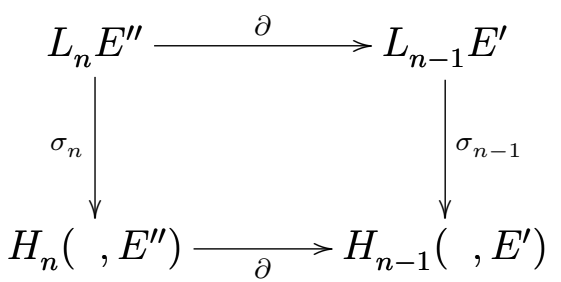

corresponding to a $\mathbf{G}$-exact sequence $0 \longrightarrow E^{\prime} \longrightarrow E \longrightarrow E^{\prime \prime} \longrightarrow 0$ commutes.

We prove this first for $\sigma_{1}$ and $\sigma_{0}$, using what is basically the classical abelian-categories method. We are indebted to F. Ulmer for pointing it out. Form the diagram

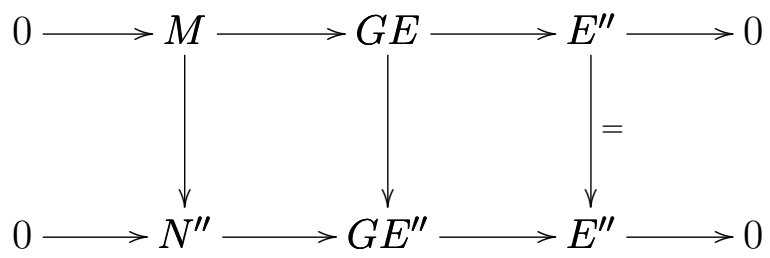

where $M$ is $\operatorname{ker}\left(G E \longrightarrow E^{\prime \prime}\right)$. The left vertical arrow exists by virtue of $N^{\prime \prime}$ being $\operatorname{ker}\left(G E^{\prime \prime} \longrightarrow E^{\prime \prime}\right)$. This induces

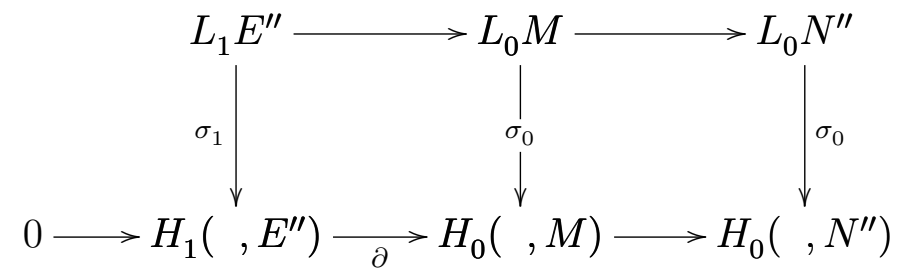

Since the map labeled $\partial$ is the kernel of $H_{0}(, M) \rightarrow H_{0}(, G E)$, there exists a map $L_{1} E^{\prime \prime} \longrightarrow H_{1}\left(, E^{\prime \prime}\right)$ such that the left square commutes. As the right square commutes by naturality of $\sigma_{0}$, the outer rectangle commutes when this unknown map $L_{1} E^{\prime \prime} \longrightarrow H_{1}\left(, E^{\prime \prime}\right)$ is inserted. But this is exactly the property which determines $\sigma_{1}$ 
uniquely. Thus the left square commutes with $\sigma_{1}$ put in. As there is obviously a map

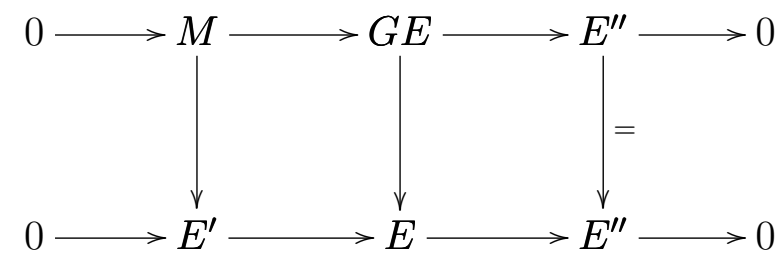

a prism is induced:

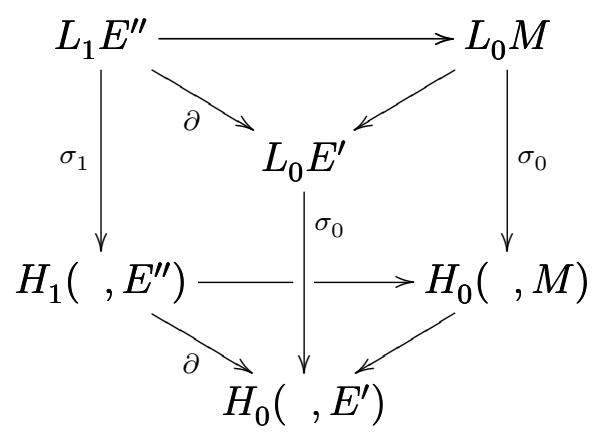

The top and bottom commute by naturality of the connecting homomorphisms in the Land $H\left(\right.$, )-theories, the right front face commutes by naturality of $\sigma_{0}$, the back commutes as it is the left square of 3.7, so the left front face also commutes, q.e.d.

The proof that the $\sigma_{n}$ are compatible with connecting homomorphisms in dimensions $>1$ is similar.

Finally, assuming that the theory $\mathbf{L}$ also satisfies the acyclicity condition 2. (or 3.1):

$$
\begin{aligned}
& L_{0}(G E) \stackrel{\cong}{\lambda} G E, \\
& L_{n}(G E)=0, \quad n>0,
\end{aligned}
$$

then from 3.5, $\sigma_{0}: L_{0} E \stackrel{\cong}{\longrightarrow} H_{0}(, E)$, and inductively from $3.7, \sigma_{n}: L_{n} E \stackrel{\cong}{\longrightarrow} H_{n}(, E)$ for $n>0$. This completes the uniqueness proof, except for Lemma 3.4.

Let $0 \longrightarrow N \longrightarrow G E \longrightarrow E \longrightarrow 0$ be exact, as above, and let $\nu: G^{2} E \longrightarrow N$ be defined by the kernel property:

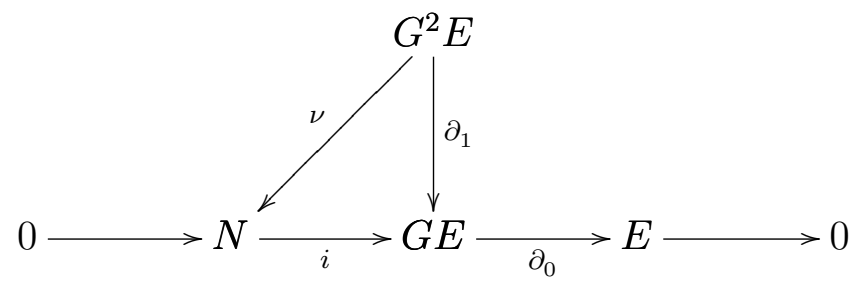


Then

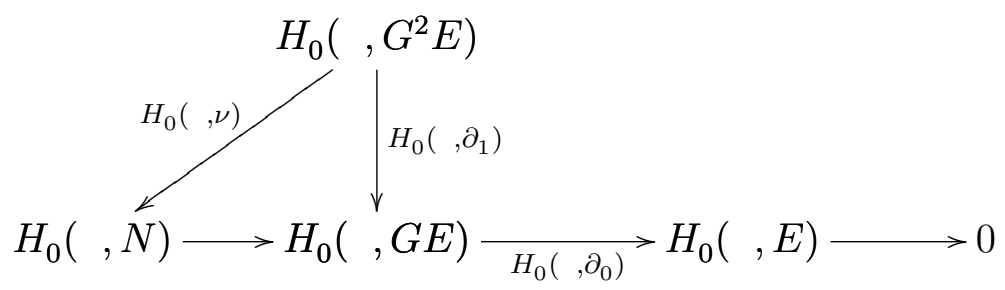

has an exact bottom row as $0 \longrightarrow N \longrightarrow \cdots$ is $\mathbf{G}$-exact as well. To prove $H_{0}\left(, \partial_{1}\right)$ and $H_{0}\left(, \partial_{0}\right)$ exact it suffices to prove that $H_{0}(, \nu)$ is onto. Let $K=\operatorname{ker} \nu$, so that $0 \longrightarrow K \longrightarrow G^{2} E \longrightarrow N$ is exact. Composing this with $G$, it is enough to show that

$$
0 \longrightarrow G K \longrightarrow G G^{2} E \stackrel{G \nu}{\longrightarrow} G N \longrightarrow 0
$$

is exact, which just means $G \nu$ onto (in fact it turns out to be split). If we apply $G$ to 3.8 , we get

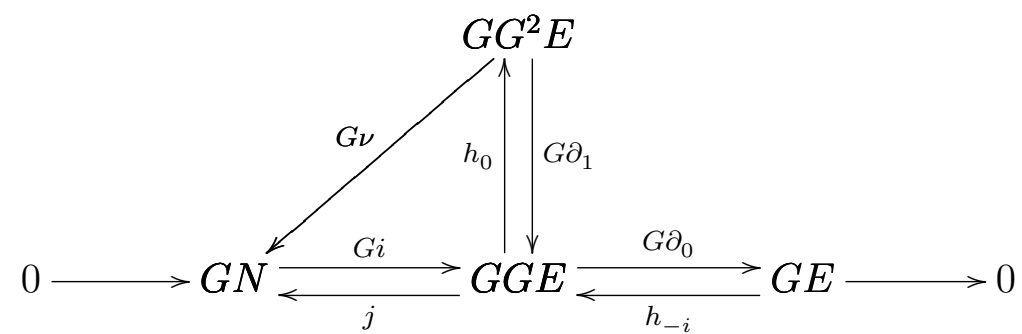

where the contracting maps $h_{-1}$ and $h_{0}$ obey $G \partial_{0} \cdot h_{-1}+h_{0} \cdot G \partial_{1}=G G E$, among other things, and the bottom row is split $\left(h_{-1}=\delta E\right.$ and $\left.h_{0}=\delta G E\right)$. Now $G \nu$ splits, for $G i \cdot h_{0} \cdot G \nu=G N$. Since $G i$ is a monomorphism it suffices to prove $G i \cdot h_{0} \cdot G \nu \cdot G i=G i$. But

$$
\begin{aligned}
G i \cdot h_{0} \cdot G(\nu i) & =G i \cdot h_{0} \cdot G \partial_{1} \\
& =G i \cdot\left(G G E-G \partial_{0} \cdot h_{-1}\right) \\
& =G i-G\left(i \partial_{0}\right) \cdot h_{-1} \\
& =G i
\end{aligned}
$$

since $i \partial_{0}$ is zero.

\section{Homology in additive categories}

Now we assume that $\mathbf{C}$ is an additive category and $\mathbf{G}=(G, \varepsilon, \delta)$ is a cotriple in $\mathbf{C}$. It is not necessary to suppose that $G: \mathbf{C} \longrightarrow \mathbf{C}$ is additive or even that $0 G=0$.

If $E: \mathbf{C} \longrightarrow \mathscr{A}$ is a coefficient functor, and this is assumed to be additive, the homology functors $H_{n}(, E)_{\mathbf{G}}: \mathbf{C} \longrightarrow \mathscr{A}$ are defined as before, and are additive. They admit of an axiomatic characterization like that in homological algebra (cf. 4.5). 
G-projectives play a big role in the additive case. We recall $P \in \mathbf{C}$ is $\mathbf{G}$-projective if there is a map $s: P \longrightarrow P G$ such that $s \cdot P \varepsilon=P$. A useful fact, holding in any category, is that the coproduct $P * Q$ of $\mathbf{G}$-projectives is again $\mathbf{G}$-projective.

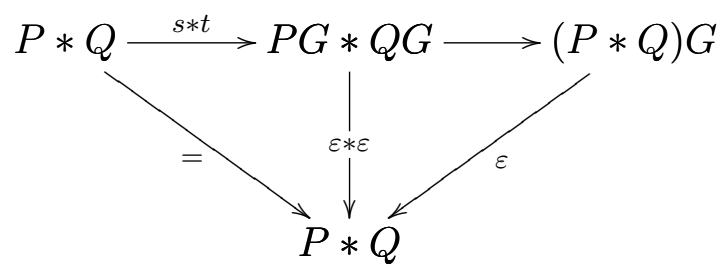

In an additive category the coproduct is $P \oplus Q$. We assume from now on that $\mathbf{C}$ is additive.

DEFINITION 4.1.

$$
X^{\prime} \stackrel{i}{\longrightarrow} X \stackrel{j}{\longrightarrow} X^{\prime \prime}
$$

is G-exact (G-acyclic) if ij $=0$ and $\left(A G, X^{\prime}\right) \longrightarrow(A G, X) \longrightarrow\left(A G, X^{\prime \prime}\right)$ is an exact sequence of abelian groups for all $A \in \mathbf{C}$, or equivalently, if $i j=0$ and

$$
\left(P, X^{\prime}\right) \longrightarrow(P, X) \longrightarrow\left(P, X^{\prime \prime}\right)
$$

is an exact sequence of abelian groups for every $\mathbf{G}$-projective $P$. A G-resolution of $X$ is a sequence $0 \longleftarrow X \longleftarrow X_{0} \longleftarrow X_{1} \longleftarrow \cdots$ which is $\mathbf{G}$-acyclic and in which $X_{0}, X_{1}, \ldots$ are G-projective.

The usual facts about $\mathbf{G}$-resolutions can be proved:

4.2 Existence And COMPARISOn TheOrem. G-resolutions always exist. If

$$
0 \longleftarrow X \longleftarrow X_{0} \longleftarrow X_{1} \longleftarrow \cdots
$$

is a $\mathbf{G}$-projective complex and $0 \longleftarrow Y \longleftarrow Y_{0} \longleftarrow Y_{1} \longleftarrow \cdots$ is a $\mathbf{G}$-acyclic complex then any $f: X \longrightarrow Y$ can be extended to a map of complexes

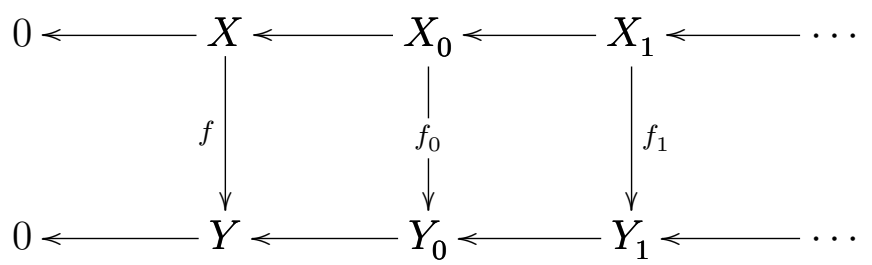

Any two such extensions are chain homotopic.

In fact,

$$
0 \longleftarrow X \longleftarrow \stackrel{\partial_{0}}{\longleftarrow} X G \ll \stackrel{\partial_{1}}{\longleftarrow} X G^{2} \longleftarrow \cdots
$$

is a $\mathbf{G}$-resolution of $X$ if we let $\partial_{n}=\sum(-1)^{i} X \varepsilon_{i}$. It is a $\mathbf{G}$-projective complex, and if $A G$ is hommed into the underlying augmented simplicial object $X G^{*}, X G^{*}$ ? the resulting 
simplicial set has a contraction $(A G, X) \stackrel{h_{-1}}{\longrightarrow}(A G, X G) \stackrel{h_{0}}{\longrightarrow}\left(A G, X G^{2}\right) \stackrel{h_{1}}{\longrightarrow} \cdots$ defined by $x \cdot h_{n}=A \delta \cdot x G$ for $x: A G \longrightarrow X G^{n+1}$. Thus the simplicial group $\left(A G, X G^{*}\right)$ has no homotopy, or homology, with respect to the boundary operators $\left(A G, \partial_{n}\right)$.

The rest of the comparison theorem is proved just as in homological algebra.

Now we characterize the homology theory $H_{n}(, E)_{\mathbf{G}}: \mathbf{C} \longrightarrow \mathscr{A}$ by axioms on the $\mathbf{C}$ variable. In doing this we use finite projective limits in $\mathbf{C}$, although we still refrain from assuming $\mathbf{G}$ additive. We do assume that the coefficient functor $E: \mathbf{C} \longrightarrow \mathscr{A}$ is additive, which forces additivity of the homology functors. The axioms we get are:

\subsection{G-ACYCLicity. If $P$ is $\mathbf{G}$-projective, then}

$$
\begin{aligned}
& H_{0}(P, E)_{\mathbf{G}} \stackrel{\cong}{\cong} P E, \\
& H_{n}(P, E)_{\mathbf{G}}=0, \quad n>0 .
\end{aligned}
$$

4.4. G-connectedness. If $0 \longrightarrow X^{\prime} \longrightarrow X \longrightarrow X^{\prime \prime} \longrightarrow 0$ is a $\mathbf{G}$-exact sequence in $\mathbf{C}$, then there is a long exact sequence in homology:

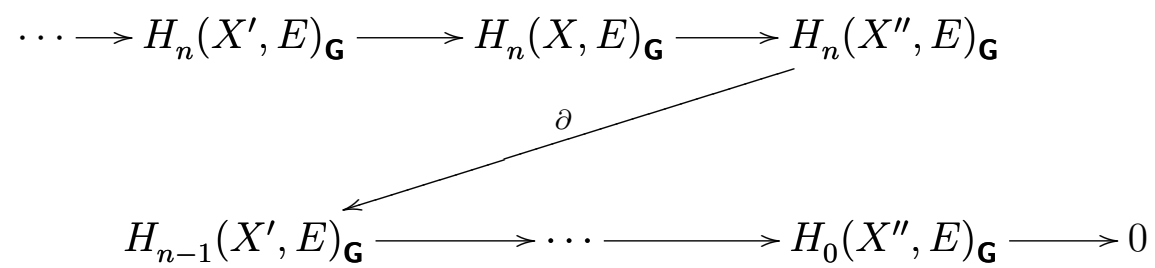

The connecting maps are natural with respect to maps of $\mathbf{G}$-exact sequences

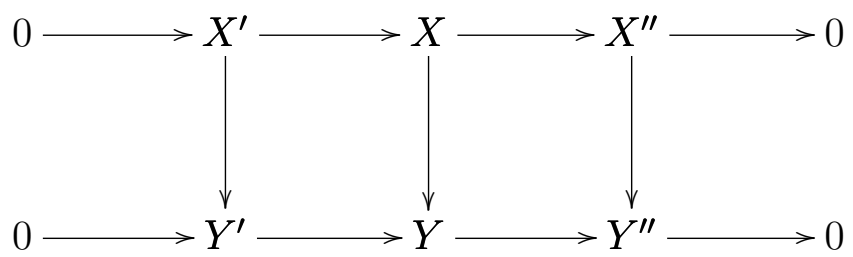

It follows from 4.4 that if $X=X^{\prime} \oplus X^{\prime \prime}$, then the canonical map

$$
H_{n}\left(X^{\prime}, E\right)_{\mathbf{G}} \oplus H_{n}\left(X^{\prime \prime}, E\right)_{\mathbf{G}} \longrightarrow H_{n}\left(X^{\prime} \oplus X^{\prime \prime}, E\right)_{\mathbf{G}}
$$

is an isomorphism, $n \geq 0$. Thus the $H_{n}(, E)_{\mathbf{G}}$ are additive functors.

We are able to prove the following characterization:

4.5 UNIQUENESS. If $E_{0} \stackrel{\lambda}{\longrightarrow} E$ is a natural transformation, and $E_{1}, E_{2}, \ldots, \partial$ is a sequence of functors together with a family of connecting homomorphisms satisfying 4.3 and 4.4, then there is a unique isomorphism of connected sequences $\sigma_{n}: E_{n} \cong H_{n}(, E)_{\mathbf{G}}, n \geq 0$, which commutes with the augmentations $E_{0} \longrightarrow E$ and $H_{0}(, E)_{\mathbf{G}} \longrightarrow E$.

For the proofs of the above, $4.3=2.1$. For 4.4 , we assume that $\mathbf{C}$ has splitting idempotents. This causes no difficulty as $\mathbf{G}$ can clearly be extended to the idempotent 
completion of $\mathbf{C}$ and any abelian category valued functor can be likewise extended [Freyd (1964)]. Moreover, it is clear that this process does not affect the derived functors. (Or assume that $\mathbf{C}$ has kernels.)

Now if $0 \longrightarrow X^{\prime} \longrightarrow X \longrightarrow X^{\prime \prime} \longrightarrow 0$ is $\mathbf{G}$-exact it follows from exactness of

$$
\left(X^{\prime \prime} G, X\right) \longrightarrow\left(X^{\prime \prime} G, X^{\prime \prime}\right) \longrightarrow 0
$$

that there is a map $X^{\prime \prime} G \longrightarrow X$ whose composite with $X \longrightarrow X^{\prime \prime}$ is $X^{\prime \prime} \varepsilon$. Applying $G$ to it we have $X^{\prime \prime} G \stackrel{X^{\prime \prime} \delta}{\longrightarrow} X^{\prime \prime} G^{2} \longrightarrow X G$ which splits $X G \longrightarrow X^{\prime \prime} G$. By our assumption we can find $X_{0}$ so that

$$
0 \longrightarrow X_{0} \longrightarrow X G \longrightarrow X^{\prime \prime} G \longrightarrow 0
$$

is split exact. $X_{0}$ being presented as a retract of a free is $\mathbf{G}$-projective. Also, the composite

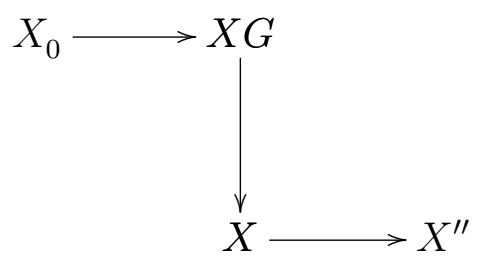

is zero and we can find $X_{0} \longrightarrow X^{\prime}$ so that

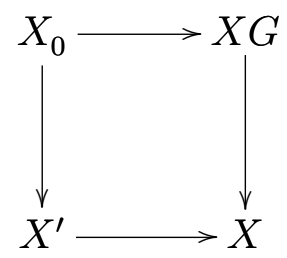

commutes. Continuing in this fashion we have a weakly split exact sequence of complexes

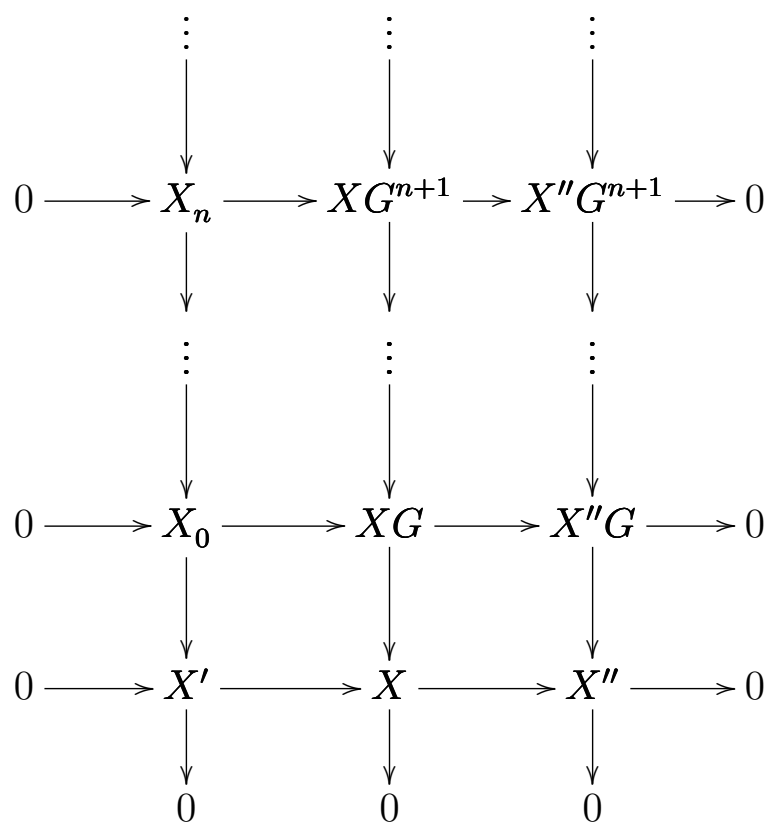


Homming a $Y G$ into it produces a weakly split exact sequence of abelian group complexes, two of which are exact, and so, by the exactness of the homology triangle, is the third. But then the first column is a $\mathbf{G}$-projective resolution of $X^{\prime}$ and the result easily follows.

For uniqueness, 4.5, $\mathrm{C}$ must have kernels, and the argument follows the classical prescription (Section 3). This i s reasonable, for otherwise there wouldn't be enough exact sequences for 4.4 to be much of a restriction. First, $X G^{2} E_{0} \longrightarrow X G E_{0} \longrightarrow X E_{0} \longrightarrow 0$ is exact in $\mathscr{A}$. Using $\lambda: E_{0} \longrightarrow E$ one gets a unique $\sigma_{0}: E_{0} \longrightarrow H_{0}(, E)$ which is compatible with the augmentations:

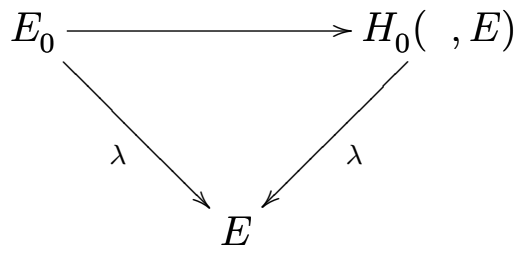

Letting $N=\operatorname{ker}(X G \longrightarrow X)$, the sequence $0 \longrightarrow N \longrightarrow X G \longrightarrow X \longrightarrow 0$ is $\mathbf{G}$-exact. $\sigma_{1}: E_{1} \longrightarrow H_{1}(, E)$ is uniquely determined by

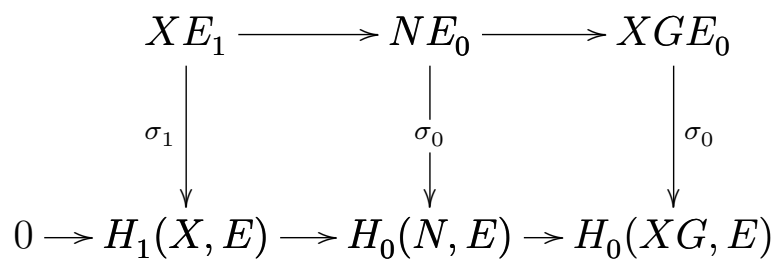

$\sigma_{n}$ similarly. Now the argument of the uniqueness part of 3.3 goes through and shows that the $\sigma$ 's commute with all connecting maps. Finally, if the $E_{n}$ are $\mathbf{G}$-acyclic (4.3), the $\sigma_{n}$ are isomorphisms.

As examples we cite $\operatorname{Tor}_{n}^{R}(A, M)$ and $\operatorname{Ext}_{R}^{n}(M, A)$ obtained as G-derived functors, or G-homology, of the coefficient functors

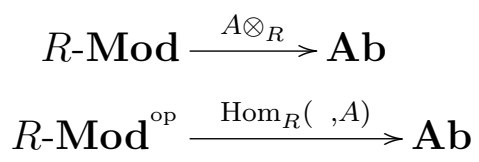

relative to the free $R$-module cotriple (1.1). Proved in this section are additivity of these functors and their usual axiomatic characterizations.

Similarly one gets axioms for the $K$-relative Tor and Ext (1.1), and for the pure Tor and Ext defined in Section 10.

4.6 Application to Section 3. Let $\mathbf{C}$ be arbitrary, G a cotriple in $\mathbf{C}$. Let $\mathbf{G}$ operate in the functor category $(\mathbf{C}, \mathscr{A})$ by composition. The resulting cotriple is called $(\mathbf{G}, \mathscr{A})$ :

$$
\begin{aligned}
& (E)(G, \mathscr{A})=G E \stackrel{(E)(\varepsilon, \mathscr{A})=\varepsilon E}{\longrightarrow} E, \\
& (E)(G, \mathscr{A})=G E \stackrel{(E)(\delta, \mathscr{A})=\delta E}{\longrightarrow} G^{2} E=(E)(G, \mathscr{A})^{2} .
\end{aligned}
$$


Iterating this cotriple in the usual way, we build up a simplicial functor

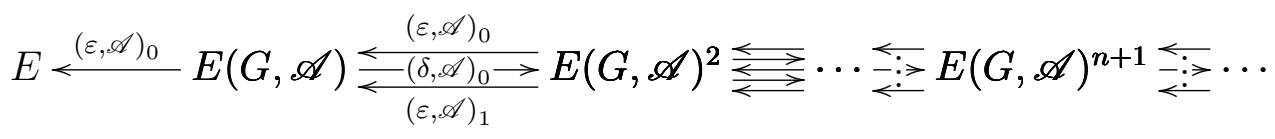

from $\mathbf{C} \longrightarrow \mathscr{A}$. Rewritten, this is

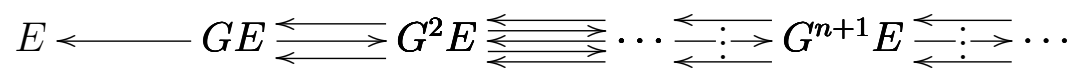

Note that the $i$-th operator $(\varepsilon, \mathscr{A})_{i}:(E)(G, \mathscr{A})^{n+1} \longrightarrow(E)(G, \mathscr{A})^{n}$ is actually $\varepsilon_{n-i} E$ using the notation of the Introduction (dual spaces cause transposition). But reversing the numbering of face and degeneracy operators in a simplicial object does not change homotopy or homology. Therefore

$$
H_{n}(E, \mathrm{id})_{(\mathbf{G}, \mathscr{A})}=H_{n}(, E)_{\mathbf{G}}, \quad n \geq 0 ;
$$

on the left coefficients are in the identity functor $(\mathbf{C}, \mathscr{A}) \longrightarrow(\mathbf{C}, \mathscr{A})$.

Thus the homology theory $H(, E)_{\mathbf{G}}$ can always be obtained from a cotriple on an additive (even abelian) category, and the cotriple can be assumed additive. How can the axioms of this section be translated into axioms for the $H_{n}(, E)_{\mathbf{G}}$ in general?

The $(\mathbf{G}, \mathscr{A})$-projective functors are just the retracts of functors of the form $G E$. Thus the acyclicity axiom 4.3 becomes:

$$
\begin{aligned}
& H_{0}(, E)_{\mathbf{G}} \stackrel{\cong}{\lambda} \text {. } \\
& H_{n}(, E)_{\mathbf{G}}=0, \quad n>0,
\end{aligned}
$$

if $E$ is $(\mathbf{G}, \mathscr{A})$-projective; this is equivalent to 3.1 .

For the homology sequence, $0 \longrightarrow E^{\prime} \longrightarrow E \longrightarrow E^{\prime \prime} \longrightarrow 0$ will be $(\mathbf{G}, \mathscr{A})$-exact if and only if $0 \longrightarrow G E^{\prime} \longrightarrow G E \longrightarrow G E^{\prime \prime} \longrightarrow 0$ is split exact in the functor category. (Prove this considering the picture

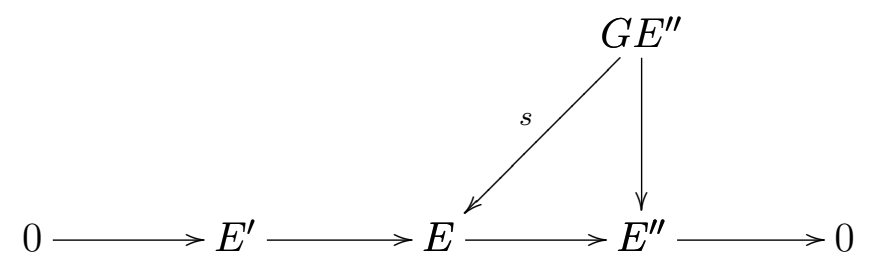

$G E^{\prime \prime} \stackrel{\delta E^{\prime \prime}}{\longrightarrow} G^{2} E^{\prime \prime} \stackrel{G s}{\longrightarrow} G E$ splits the sequence. $) \quad(\mathbf{G}, \mathscr{A})$-exactness $\Longrightarrow$ G-exactness as defined in 3.2. The homology sequence axiom of this section is weaker than that of Section 3: it requires the exact homology sequence to be produced for a smaller class of short exact sequences.

Concepts equivalent to $(\mathbf{G}, \mathscr{A})$-projectivity and -exactness have recently been employed by Mac Lane to give a projective complex $\rightarrow$ acyclic complex form to the cotriple acyclic-models comparison theorem 11.1 (unpublished). In particular, G-representability in the acyclic-models sense (existence of $\vartheta: E \longrightarrow G E$ splitting the counit $\varepsilon E: G E \longrightarrow E$ ) is the same thing as $(\mathbf{G}, \mathscr{A})$-projectivity, $\mathbf{G}$-contractibility is the same as $(\mathbf{G}, \mathscr{A})$-acyclicity. 
4.7 Applichtion to extensions. Let an $n$-dimensional G-extension of $X$ by $Y$ be a $\mathbf{G}$-exact sequence

$$
0 \longrightarrow Y \longrightarrow X_{n-1} \longrightarrow \cdots \longrightarrow X_{0} \longrightarrow X \longrightarrow 0, \quad n>0 .
$$

Under the usual Yoneda equivalence these form a set $E^{n}(X, Y)_{\mathbf{G}} \cdot E^{0}(X, Y)_{\mathbf{G}}=(X, Y)$, the hom set in $\mathbf{C}$ (which is independent of $\mathbf{G}$ ). Using the comparison theorem 4.2, an extension gives rise to a map of complexes

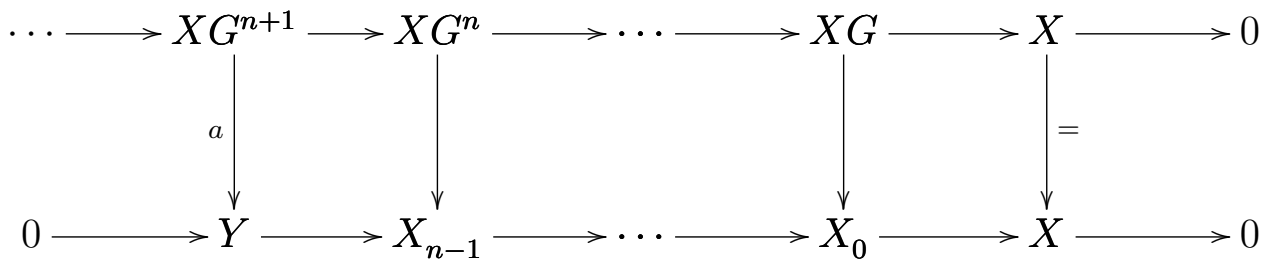

The map $a$ is an $n$-cocycle of $X$ with values in the representable functor $(, Y): \mathbf{C}^{\mathrm{op}} \longrightarrow \mathbf{A b}$. We get in this way a map

$$
E^{n}(X, Y)_{\mathbf{G}} \longrightarrow H^{n}(X, Y)_{\mathbf{G}}, \quad n \geq 0
$$

(in dimension 0 , any $X \longrightarrow Y$ determines a 0-cocycle $X G \longrightarrow X \longrightarrow Y$ ).

In practice cotriples often have the property that $X G^{2} \rightleftarrows X G \longrightarrow X$ is always a coequalizer diagram. In this case,

$$
E^{n}(X, Y)_{\mathbf{G}} \longrightarrow H^{n}(X, Y)_{\mathbf{G}}
$$

is an isomorphism for $n=0$, and a monomorphism for $n>0$. If $\mathbf{G}$ is the free cotriple in a tripleable adjoint pair $\mathbf{C} \longrightarrow \mathscr{A} \longrightarrow \mathbf{C}$ this coequalizer condition holds; in fact, in that case

$$
E^{n}(X, Y)_{\mathbf{G}} \stackrel{\cong}{\longrightarrow} H^{n}(X, Y)_{\mathbf{G}}, \quad n \geq 0,
$$

as is proved in [Beck (1967)].

In categories of modules or abelian categories with projective generators [Huber (1962)], this gives the usual cohomological classification of extensions.

\section{General notion of a $\mathbf{G}$-resolution and the fact that the homology depends} on the $\mathbf{G}$-projectives alone

There is no shortage of resolutions from which the G-homology can in principle be computed, as the standard one always exists. But it would be nice to be able to choose more convenient resolutions in particular problems, and have available something like the additive comparison theorem (Section 4) in order to relate them to the standard resolutions. In fact a simplicial comparison theorem does exist [Kleisli (1967)], but we can get by with something much easier. Any category can be made freely to generate an additive category 
by a well known construction and we find the solution to our problem by transferring it to this additive context. This is the same technique as is used by André [André (1967), Section 4].

The free additive category on $\mathbf{C}, \mathbf{Z C}$, has formal sums and differences of maps in $\mathbf{C}$ as its maps. Exact definitions and properties connected with $\mathbf{Z C}$ are given after the following definitions.

5.1 Definitions. A G-resolution of $X$ is a complex

$$
X \stackrel{\partial_{0}}{\longleftarrow} X_{0} \longleftarrow \partial_{1} X_{1} \longleftarrow \cdots \stackrel{\partial_{n}}{\longleftarrow} X_{n} \longleftarrow \cdots
$$

in $\mathbf{Z C}$ in which all $X_{n}$ for $n \geq 0$ are $\mathbf{G}$-projective and which is $\mathbf{G}$-acyclic in the sense that

$$
0 \longleftarrow(A G, X)_{\mathbf{z C}} \longleftarrow\left(A G, X_{0}\right)_{\mathbf{z C}} \longleftarrow \cdots \longleftarrow\left(A G, X_{n}\right)_{\mathbf{Z C}} \longleftarrow \cdots
$$

has zero homology in all dimensions for all values $A G$ of the cotriple $\mathbf{G}$.

A simplicial G-resolution of $X$ is an augmented simplicial object in $\mathbf{C}$

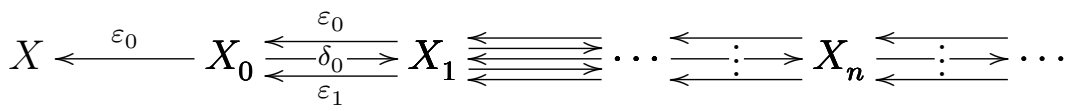

$$
\begin{aligned}
& X \stackrel{\partial_{0}=\left(\varepsilon_{0}\right)}{\longleftarrow} X_{0} \stackrel{\partial_{1}=\left(\varepsilon_{0}\right)-\left(\varepsilon_{1}\right)}{\longleftarrow} X_{1} \longleftarrow \cdots \stackrel{\partial_{n}}{\longleftarrow} X_{n} \longleftarrow \cdots
\end{aligned}
$$

is a G-resolution as defined above (as usual, $\partial_{n}=\sum(-1)^{i}\left(\varepsilon_{i}\right)$ ). In particular, the standard complex

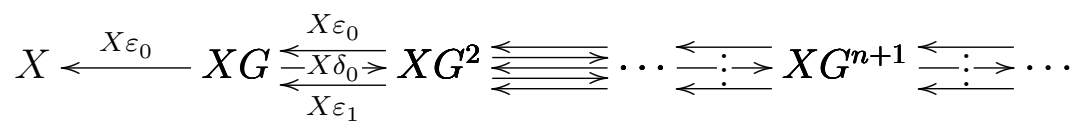

is a $\mathbf{G}$-resolution of $X$, since the simplicial set $\left(A G, X G^{n+1}\right)_{n \geq-1}$ has the contraction given in the proof of 4.2 .

To be precise about $\mathbf{Z C}$, its objects are the same as those of $\mathbf{C}$, while a map $X \longrightarrow Y$ in $\mathbf{Z C}$ is a formal linear combination of such maps in $\mathbf{C}$, i.e., if $n_{i} \in \mathbf{Z}$ and $f_{i}: X \longrightarrow Y \in \mathbf{C}$, we get a map

$$
X \stackrel{\sum n_{i}\left(f_{i}\right)}{\longrightarrow} Y
$$

in ZC. (We enclose the free generators in parentheses for clarity in case $\mathbf{C}$ is already additive.) Composition is defined like multiplication in a group ring, $\left(\sum m_{i}\left(f_{i}\right)\right)\left(\sum n_{j}\left(g_{j}\right)\right)=$ $\sum \sum m_{i} n_{j}\left(f_{i} g_{j}\right)$.

The natural inclusion of the basis $\mathbf{C} \longrightarrow \mathbf{Z C}$ can be used to express the following universal mapping property. If $E: \mathbf{C} \longrightarrow \mathscr{A}$ is a functor into an additive category, there is a unique additive functor $\bar{E}: \mathbf{Z C} \longrightarrow \mathscr{A}$ such that

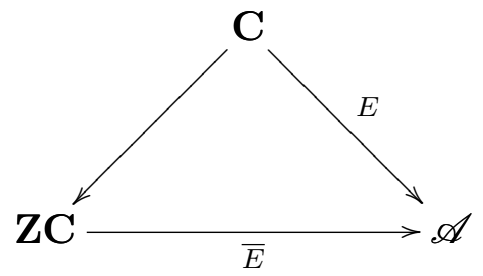


commutes. Explicitly, $X \bar{E}=X E$ and $\left(\sum n_{i}\left(f_{i}\right)\right) \bar{E}=\sum n_{i} f_{i} E$.

Let $\mathbf{G}=(G, \varepsilon, \delta)$ be a cotriple in $\mathbf{C}$. Thinking of $G$ as taking values in $\mathbf{Z C}$ we get an additive extension

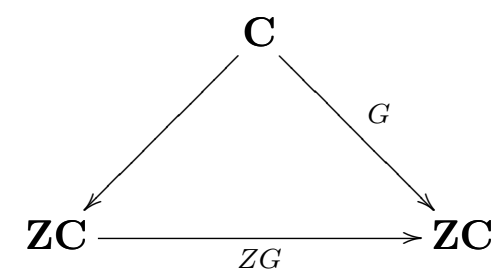

which is a cotriple $Z \mathbf{G}=(Z G, Z \varepsilon, Z \delta)$ in ZC. Explicitly, $X \cdot Z G=X G$, and the counit and comultiplication are

$$
\begin{aligned}
& X G \stackrel{(X \varepsilon)}{\longrightarrow} X, \\
& X G \stackrel{(X \delta)}{\longrightarrow} X G^{2} .
\end{aligned}
$$

Although there are more maps in $\mathbf{Z C}$, the notion of object does not change, and neither does the notion of projective object. For $P \in \mathbf{C}$ is $\mathbf{G}$-projective $\Leftrightarrow P$ regarded as an object in $\mathbf{Z C}$ is $Z \mathbf{G}$-projective. The forward implication is evident, and if

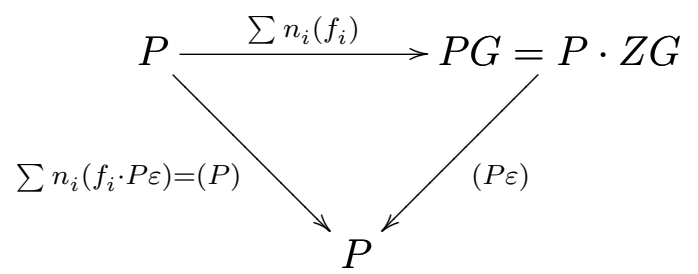

then $f_{i} \cdot P \varepsilon=P$ for some $i$, as $(P, P)_{\mathbf{z C}}$ is a free abelian group on a basis of which both $f_{i} \cdot P \varepsilon$ and $P$ are members; this proves the other implication.

Thus the $\mathbf{G}$-resolutions of 5.1 are exactly the $Z \mathbf{G}$-resolutions relative to the cotriple in the additive category $\mathbf{Z C}$, in the sense of 4.1. Invoking the comparison theorem 4.2 , we see that if $\left(X_{n}\right)$ and $\left(Y_{n}\right)$ are $\mathbf{G}$ - or equivalently $Z \mathbf{G}$-resolutions of $X_{-1}=Y_{-1}=X$, then there is a chain equivalence

$$
\left(X_{n}\right) \stackrel{\cong}{\longrightarrow}\left(Y_{n}\right)
$$

in $\mathbf{Z C}$.

Finally, let $E: \mathbf{C} \longrightarrow \mathscr{A}$ be a coefficient functor and $\bar{E}: \mathbf{Z C} \longrightarrow \mathscr{A}$ its additive extension constructed above. As the following complexes are identical:

$$
\begin{gathered}
X(Z G) \bar{E} \stackrel{\partial_{1}}{\longleftarrow} X(Z G)^{2} \bar{E} \longleftarrow \cdots \stackrel{\partial_{n}=\left(\sum_{0}^{n}(-1)^{i}\left(X \varepsilon_{i}\right)\right) \bar{E}}{\longleftarrow} X(Z G)^{n+1} \bar{E} \longleftarrow \cdots \\
X G E \longleftarrow \partial_{1} X G^{2} E \longleftarrow \cdots \stackrel{\partial_{n}=\sum_{0}^{n}(-1)^{i} X \varepsilon_{i} E}{\longleftarrow} X G^{n+1} E \longleftarrow \cdots
\end{gathered}
$$

we conclude that

$$
H_{n}(X, E)_{\mathbf{G}}=H_{n}(X, \bar{E})_{\mathbf{Z G}}, \quad n \geq 0,
$$


another reduction of the general homology theory to the additive theory of Section 4 . The last equation states that the diagram

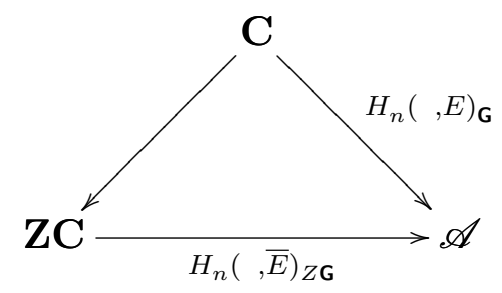

commutes, that is, the $H_{n}(, \bar{E})_{\mathbf{Z G}}$ are the additive extensions of the $H_{n}(, E)_{\mathbf{G}}$.

Parenthetically, an additive structure on $\mathbf{C}$ is equivalent to a unitary, associative functor $\vartheta: \mathbf{Z C} \longrightarrow \mathbf{C}$, that is, $Z()$ is a triple in the universe, and its algebras are the additive categories; if $\mathbf{C}$ is additive, $\vartheta$ is $\left(\sum n_{i}\left(f_{i}\right)\right) \vartheta=\sum n_{i} f_{i}$.

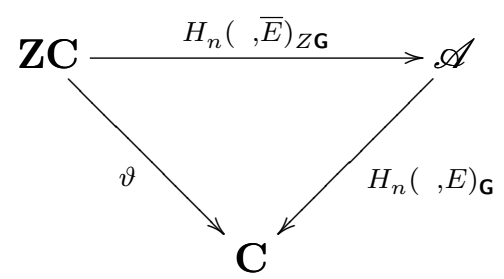

also commutes. In fact, this commutativity relation is equivalent to additivity of the homology functors, which in turn is equivalent to the homology functors' being $Z$ ( )algebra maps.

In the general case $-\mathbf{C}$ arbitrary - the above gives the result that $\mathbf{G}$-homology depends only on the $\mathbf{G}$-projectives:

THEOREM (5.2). Let $\mathbf{G}$ and $\mathbf{K}$ be cotriples in $\mathbf{C}$ such that the classes of $\mathbf{G}$-projectives and $\mathbf{K}$-projectives coincide. Then $\mathbf{G}$ and $\mathbf{K}$ determine the same homology theory, that is, there is an isomorphism

$$
H_{n}(X, E)_{\mathbf{G}} \stackrel{\cong}{\longrightarrow} H_{n}(X, E)_{\mathbf{K}}, \quad n \geq 0,
$$

which is natural in both variables $X \in \mathbf{C}, E \in(\mathbf{C}, \mathscr{A})$.

The same isomorphism holds for homology groups of a map $X \longrightarrow Y$ (see Section 2).

If $(\mathbf{G}, X)$ and $(\mathbf{K}, X)$ are $\mathbf{G}$ and $\mathbf{K}$ lifted to the category of objects over $X,(\mathbf{C}, X)$, then the $(\mathbf{G}, X)$ - and $(\mathbf{K}, X)$-projectives also coincide. Thus if $E:(\mathbf{C}, X) \longrightarrow \mathscr{A}$ is a coefficient functor, there is an isomorphism

$$
H_{n}(W, E)_{(\mathbf{G}, X)} \stackrel{\cong}{\longrightarrow} H_{n}(W, E)_{(\mathbf{K}, X)}, \quad n \geq 0,
$$

natural with respect to the variables $W \longrightarrow X \in(\mathbf{C}, X)$ and $E:(\mathbf{C}, X) \rightarrow \mathscr{A}$. 
Proof. $P \in \mathbf{C}$ is $\mathbf{Z G}$-projective $\Leftrightarrow P$ is $Z \mathbf{K}$-projective. The augmented complexes

$$
\begin{aligned}
& X \longleftarrow X G \longleftarrow X G^{2} \longleftarrow \cdots \\
& X \longleftarrow X K \longleftarrow X K^{2} \longleftarrow \cdots
\end{aligned}
$$

in $\mathbf{Z C}$ are thus projective and acyclic with respect to the same projective class in $\mathbf{Z C}$. The comparison theorem yields a chain equivalence

$$
\left(X G^{n+1}\right) \stackrel{\cong}{\longrightarrow}\left(X K^{n+1}\right)_{n \geq-1}
$$

As to naturality in $X$, if $X \longrightarrow X_{1}$ in $\mathbf{C}$, the comparison theorem also says that

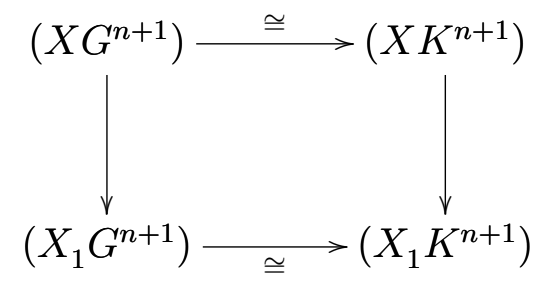

commutes up to chain homotopy. The comment about homology of a map follows from homotopy-invariance of mapping cones. $W \longrightarrow X$ being $(\mathbf{G}, X)$-projective $\Leftrightarrow W$ is $\mathbf{G}$ projective is a trivial calculation.

5.2 can also be proved through the intermediary of homology in categories with models ([Appelgate (1965)], [André (1967), Section 12], and Section 10 below), as well as by a derived-functors argument (Ulmer).

To conclude this section we state the criteria for $\mathbf{G}$-resolutions and $(\mathbf{G}, X)$-resolutions which will be used in Sections 6-9.

Proposition (5.3).

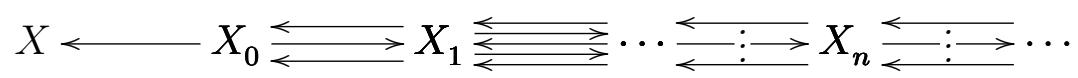

is a simplicial $\mathbf{G}$-resolution of $X=X_{-1}$ if the $X_{n}$ are $\mathbf{G}$-projective for $n \geq 0$ and the following condition, which implies $\mathbf{G}$-acyclicity, holds: the cotriple $\mathbf{G}$ factors through an adjoint pair

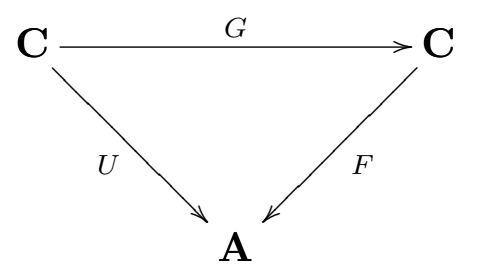

and the simplicial object $\left(X_{n} U\right)_{n \geq-1}$ in the underlying category $\mathbf{A}$ has a contraction

$$
X U \stackrel{h_{-1}}{\longrightarrow} X_{0} U \stackrel{h_{0}}{\longrightarrow} X_{1} U \stackrel{h_{1}}{\longrightarrow} \cdots \longrightarrow X_{n} U \stackrel{h_{n}}{\longrightarrow} \cdots
$$


(satisfying $h_{n} \cdot \varepsilon_{i} U=\varepsilon_{i} U \cdot h_{n-1}$ for $0 \leq i \leq n$ and $h_{n} \cdot \varepsilon_{n+1} U=X_{n} U$ ). In particular, the standard $\mathbf{G}$-resolution $\left(X G^{n+1}\right)_{n \geq-1}$ then has such a contraction:

$$
X U \stackrel{h_{-1}}{\longrightarrow} X G U \stackrel{h_{0}}{\longrightarrow} \cdots \longrightarrow X G^{n+1} U \stackrel{h_{n}}{\longrightarrow} \cdots
$$

to wit, $h_{n}=X G^{n} U \eta$ where $\eta$ is the adjointness unit $\eta: \mathbf{A} \longrightarrow F U$.

Complement. Let

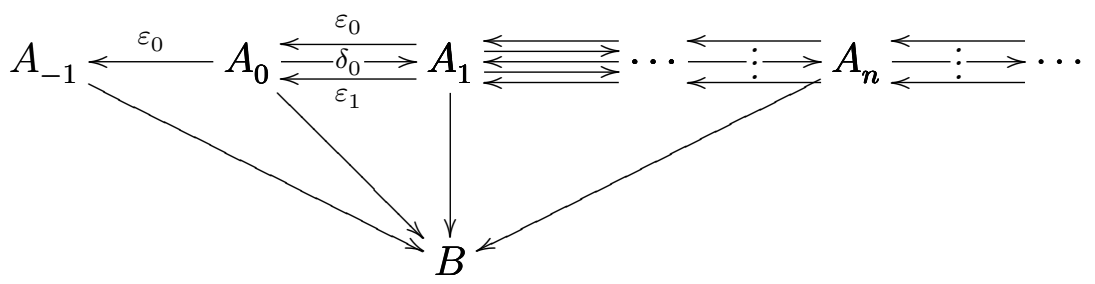

be a simplicial object in a category of objects over $B,(\mathbf{A}, B)$. If

$$
A_{-1} \stackrel{h_{-1}}{\longrightarrow} A_{0} \stackrel{h_{0}}{\longrightarrow} A_{1} \stackrel{h_{1}}{\longrightarrow} \cdots
$$

is a contraction of the simplicial object sans $B$, then it is also a contraction of the simplicial object in $(\mathbf{A}, B)$, that is, the $h_{n}$ commute with the structural maps into $B$ :

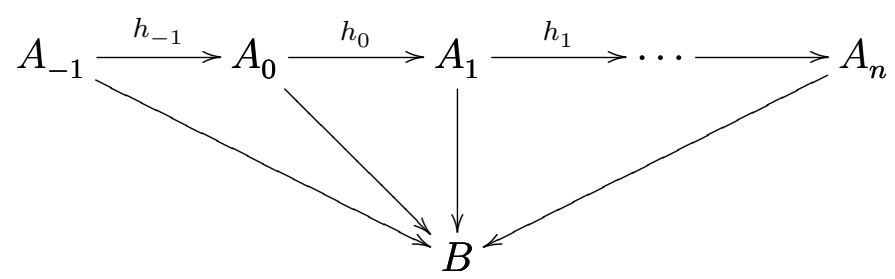

Thus when searching for contractions in categories of objects over a fixed object, the base object can be ignored.

Proof. If the stated condition holds, the simplicial set

$$
\left(A G, X_{n}\right)_{n \geq-1}
$$

has a contraction, so the free abelian group complex $\left(A G, X_{n}\right)_{\mathbf{z C}}$ has homology zero in all dimensions $\geq-1$. Indeed,

$$
\left(A G, X_{n}\right) \stackrel{k_{n}}{\longrightarrow}\left(A G, X_{n+1}\right), \quad n \geq 1,
$$

is defined by

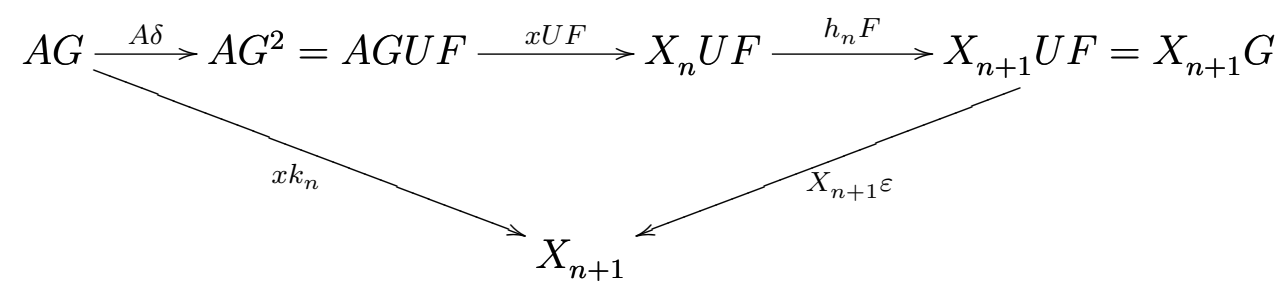


if $x: A G \longrightarrow X_{n}$.

As to the Complement, if the maps into $B$ are $p_{n}: A_{n} \longrightarrow B$ then

$$
h_{n} \cdot p_{n+1}=h_{n} \varepsilon_{n+1} p_{n}=A_{n} p_{n}=p_{n}
$$

so in view of $h_{n}$ 's satisfying the identity $h_{n} \cdot \varepsilon_{n+1}=A_{n}$, it is a map over $B$.

\section{Acyclicity and coproducts}

Given a G-resolution

$$
X=X_{-1} \longleftarrow X_{0} \leftleftarrows X_{1} \leftleftarrows
$$

is its term-by-term coproduct with a fixed object $Y$,

$$
X * Y \longleftarrow X_{0} * Y \leftleftarrows X_{1} * Y \leftleftarrows
$$

still a $\mathbf{G}$-resolution? (The new face operators are of the form $\varepsilon_{i} * Y$.) If $Y$ is $\mathbf{G}$-projective, so are all the $X_{n} * Y, n \geq 0$. The problem is, is $\mathbf{G}$-acyclicity preserved? In this section we consider the examples of groups, commutative algebras and (associative) algebras, and prove that acyclicity is preserved, sometimes using supplementary hypotheses. The cotriples involved come from adjoint functors

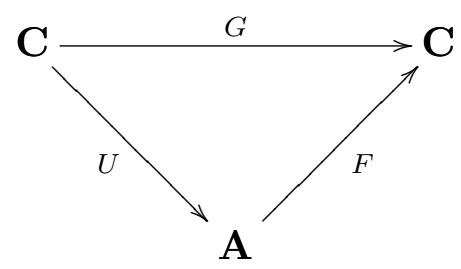

The general idea is to assume that $\left(X_{n}\right)$ has a contraction in $\mathbf{A}$ and then show that this contraction somehow induces one in $\left(X_{n} * Y\right)$, even though the coproduct $*$ is not usually a functor on the underlying category level.

6.1. Groups. Let $\left(\Pi_{n}\right)_{n \geq-1}$ be an augmented simplicial group and $U: \mathscr{G} \longrightarrow \mathscr{S}$ the usual underlying set functor where $\mathscr{G}$ is the category of groups.

From simplicial topology we know that the underlying simplicial set $\left(\Pi_{n} U\right)$ has a contraction if and only if the natural map into the constant simplicial set

$$
\left(\Pi_{n} U\right)_{n \geq 0} \longrightarrow\left(\Pi_{-1} U\right)
$$

is a homotopy equivalence if and only if the set of components of $\Pi_{*} U$ is $\Pi_{-1} U$, and $H_{n}\left(\Pi_{*} U\right)=0$ for $n>0\left(\Pi_{*}=\left(\Pi_{n}\right)_{n \geq 0}\right)$. (This is because simplicial groups satisfy the Kan extension condition, hence Whitehead's Theorem; $\pi_{1}=H_{1}$ by the group property, so the fundamental group is zero, above). 
Now suppose that $\left(\Pi_{n} U\right)_{n>-1}$ is acyclic, or has a contraction, and $\Pi$ is another group. We shall prove that $\left(\left(\Pi_{n} * \Pi\right) U\right)_{n \geq-1}$ also has a contraction;

We do this by considering the group ring functor $\mathbf{Z}(): \mathscr{G} \longrightarrow$ Rings. The simplicial ring $\mathbf{Z} \Pi_{*}$ obtained by applying the group ring functor in dimensionwise fashion has a contraction, namely the additive extension of the given set contraction in $\Pi_{*}$. In (6.3) below we shall show that the coproduct of this simplicial ring with $\mathbf{Z} \Pi$ in the category of rings, $\left(\mathbf{Z} \Pi_{*}\right) * \mathbf{Z} \Pi$, where the $n$-dimensional component is $\mathbf{Z} \Pi_{n} * \mathbf{Z} \Pi$, also has a contraction. But as the group ring functor is a left adjoint,

$$
\left(\mathbf{Z} \Pi_{*}\right) * \mathbf{Z} \Pi \stackrel{\sim}{\longrightarrow} \mathbf{Z}\left(\Pi_{*} * \Pi\right)
$$

Thus the set of components of the complex on the right is just $\mathbf{Z}\left(\Pi_{-1} * \Pi\right)$ and its $n$ th homotopy is zero for $n>0$. This implies that $\Pi_{*} * \Pi$ has $\Pi_{-1} * \Pi$ as its set of components and has no higher homotopy. (This is equivalent to the curiosity that $\mathbf{Z}($ ) as an endofunctor on sets satisfies the hypotheses of the "precise" tripleableness theorem ([Beck (1967), Theorem 1] or [Linton (1969a)].)

6.2. Commutative algebras. First let $\mathbf{G}_{A}$ be the cotriple relative to $A$-modules:

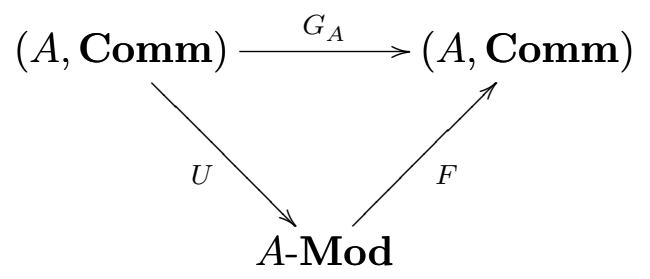

$\mathbf{G}_{A}$-resolutions behave very well with respect to coproducts of commutative $A$-algebras, $B \otimes_{A} C$. Indeed, as the standard resolution

$$
B \longleftarrow B G_{A} \leftleftarrows B G_{A}^{2 \leftleftarrows} \ldots
$$

has an A-linear contraction (5.3), so has

$$
B \otimes_{A} C \longleftarrow B G_{A} \otimes_{A} C \leftleftarrows B G_{A}^{2} \otimes_{A} C \leftleftarrows
$$

On the other hand, let $\mathbf{G}$ be the absolute cotriple

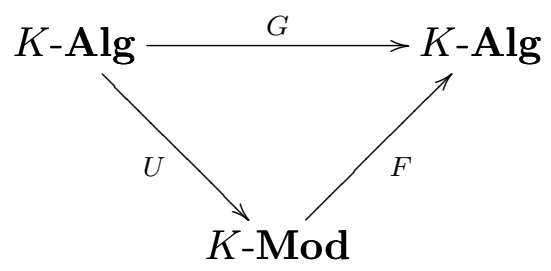

The standard G-resolution has a contraction on the underlying set level (5.3). Thus the chain complex of $A$-modules associated to

$$
B \longleftarrow B G \longleftarrow B G^{2} \leftleftarrows
$$


is an $A$-free resolution of $B$ as an $A$-module in the usual homological sense. Thus the nonnegative-dimensional part of

$$
B \otimes_{A} C \longleftarrow B G \otimes_{A} C \longleftarrow B G^{2} \otimes_{A} C \longleftarrow \leftleftarrows
$$

has $H_{n}=\operatorname{Tor}_{n}^{A}(B, C), n \geq 0$. Since it is also a group complex, this simplicial object will have a contraction as a simplicial set $\Leftrightarrow \operatorname{Tor}_{n}^{A}(B, C)=0, n>0$.

6.3. Resolutions and Coproducts of Associative Algebras. Let $K$-Alg be the category of associative $K$-algebras with identity. We are interested in resolutions relative to the adjoint pair

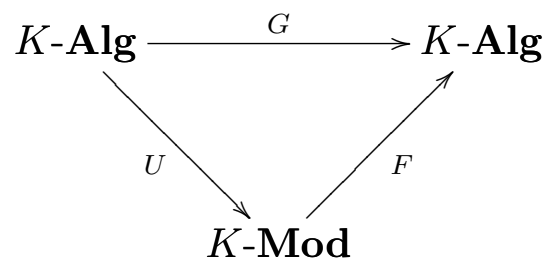

These will give rise to Hochschild homology. Here $F$ is the $K$-tensor algebra $M F=$ $K+M+M \otimes M+M \otimes M \otimes M+\cdots$. If $\Lambda, \Gamma$ are $K$-algebras, their coproduct

$$
\Lambda * \Gamma=(\Lambda+\Gamma) F / I
$$

where $I$ is the 2 -sided ideal generated by the elements

$$
\lambda_{1} \otimes \lambda_{2}-\lambda_{1} \lambda_{2}, \gamma_{1} \otimes \gamma_{2}-\gamma_{1} \gamma_{2}, 1_{K}-1_{\Lambda}, 1_{K}-1_{\Gamma}
$$

$\left(1_{K}\right.$ is in the summand of degree $0,1_{\Lambda}$ and $1_{\Gamma}$ are in the summand of degree 1$)$. The $K$-linear maps $\Lambda, \Gamma \longrightarrow(\Lambda \oplus \Gamma) F$ become algebra maps when $I$ is divided out and these two maps are the coproduct injections $\Lambda, \Gamma \longrightarrow \Lambda * \Gamma$. (In fact, $I$ is the smallest ideal which makes these maps of unitary $K$-algebras.)

Let $\left(\Lambda_{n}\right)_{n \geq-1}$ be an augmented simplicial algebra which is $U$-contractible, i.e., there exists a $K$-linear contraction

$$
\Lambda_{-1} U \stackrel{h_{-1}}{\longrightarrow} \Lambda_{0} U \stackrel{h_{0}}{\longrightarrow} \Lambda_{1} U \longrightarrow \cdots
$$

We want to know that such a contraction continues to exist in the simplicial algebra $\left(\Lambda_{n} * \Gamma\right)_{n \geq-1}$. But we can only prove this in a special case.

An algebra $\Lambda$ is called $K$-supplemented if there is a $K$-linear map $\Lambda \longrightarrow K$ such that $K \longrightarrow \Lambda \longrightarrow K$ is the identity of $K$. (The first map sends $1_{K} \longrightarrow 1_{\Lambda}$ ). An algebra map $\Lambda \longrightarrow \Lambda_{1}$ is called $K$-supplemented if $\Lambda, \Lambda_{1}$ are $K$-supplemented and

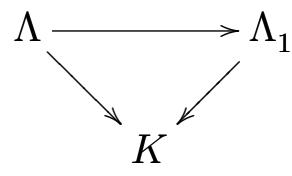


commutes.

We will show that if $\Lambda, \Gamma$ are $K$-supplemented, then the coproduct of the canonical resolution of $\Lambda$ with $\Gamma$,

$$
\Lambda * \Gamma \longleftarrow \Lambda G * \Gamma \longleftarrow \Lambda G^{2} * \Gamma £
$$

possesses a $K$-contraction. We refer to $(5.3)$ for the fact that $\left(\Lambda G^{n+1}\right)_{n \geq 1}$ always has a $K$-linear contraction, and we prove that this contraction survives into the coproduct of the resolution with $\Gamma$.

The cotriple $\mathbf{G}$ operates in a natural way in the category of $K$-supplemented algebras. For if $\Lambda$ is $K$-supplemented, the composition $\Lambda G \rightarrow \Lambda \rightarrow K$ defines a $K$-supplementation of $\Lambda G$. If $\Lambda \longrightarrow \Lambda_{1}$ is $K$-supplemented, so is the induced $\Lambda G \longrightarrow \Lambda_{1} G$,

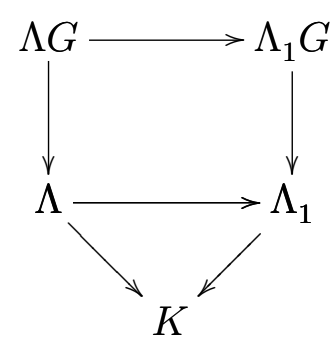

and if $\Lambda$ is $K$-supplemented, the counit and comultiplication maps $\Lambda G \longrightarrow \Lambda, \Lambda G \longrightarrow \Lambda G^{2}$ are also $K$-supplemented.

When $\Lambda$ is $K$-supplemented let $\bar{\Lambda}=\operatorname{ker}(\Lambda \longrightarrow K)$. If $f: \Lambda \longrightarrow \Lambda_{1}$ is $K$-supplemented, then $f=K \oplus \bar{f}$ where $\bar{f}: \bar{\Lambda} \longrightarrow \bar{\Lambda}_{1}$ is induced in the obvious way. This means that if we write $f: K \oplus \bar{\Lambda} \longrightarrow K \oplus \bar{\Gamma}$ in the form of $2 \times 2$ matrix, the matrix is diagonal:

$$
\left(\begin{array}{cc}
K & 0 \\
0 & f
\end{array}\right)
$$

Using the above supplementation and writing $\Lambda G^{n+1}=K \oplus \overline{\Lambda G^{n+1}}$, all of the face operators in the standard resolution $\left(\Lambda G^{n+1}\right)_{n \geq-1}$ will be diagonal:

$$
K \oplus \overline{\Lambda G^{n}} \stackrel{\left(\begin{array}{cc}
K & 0 \\
0 & \bar{\varepsilon}_{i}
\end{array}\right)}{\longleftarrow} K \oplus \overline{\Lambda G^{n+1}}, \quad 0 \leq i \leq n .
$$

The $K$-linear contraction $h_{n}: \Lambda G^{n+1} \longrightarrow \Lambda G^{n+2}$ is given by a $2 \times 2$ matrix

$$
K \oplus \overline{\Lambda G^{n+1}} \stackrel{\left(\begin{array}{ll}
h_{11} & h_{12} \\
h_{21} & h_{22}
\end{array}\right)}{\longrightarrow} K \oplus \overline{\Lambda G^{n+2}}
$$

The relation $h_{n} \varepsilon_{n+1}=\Lambda G^{n+1}$ is equivalent to

$$
h_{11}=K, \quad h_{21}=0, \quad h_{12} \bar{\varepsilon}_{n+1}=0, \quad h_{22} \bar{\varepsilon}_{n+1}=\overline{\Lambda G^{n+1}}
$$


(the matrix acts on row vectors from the right). The relation $h_{n} \varepsilon_{i}=\varepsilon_{i} h_{n}, 0 \leq i \leq n$, is equivalent to $h_{22} \bar{\varepsilon}_{i}=\bar{\varepsilon}_{i} h_{22}$ as another matrix calculation shows. Thus the contraction matrix has the form

$$
\left(\begin{array}{cc}
K & h_{12} \\
0 & h_{22}
\end{array}\right)
$$

where entry $h_{22}$ satisfies the contraction identities with respect to the restrictions of the face operators $\varepsilon_{i}$ to the supplementation kernels, i.e. with respect to the maps $\bar{\varepsilon}_{i}$. Thus, we can switch to

$$
h_{n}^{\prime}=\left(\begin{array}{cc}
K & 0 \\
0 & h_{22}
\end{array}\right)
$$

which is also a matrix representation of a $K$-linear contraction

$$
\begin{array}{rll}
\Lambda G^{n+1} & \stackrel{h_{n}^{\prime}}{\longrightarrow} & \Lambda G^{n+2} \\
K \oplus \overline{\Lambda G^{n+1}} & \stackrel{K \oplus \overline{h_{n}}}{\longrightarrow} & K \oplus \overline{\Lambda G^{n+2}}
\end{array}
$$

where we have written $\bar{h}_{n}$ in place of $h_{22}$. This change can be made for all $n \geq-1$, so we get a $K$-contraction which is in diagonal form.

The next step is to find that the coproduct of two $K$-supplemented algebras can be written in a special form. Consider the direct sum

$$
W=K+\bar{\Lambda}+\bar{\Gamma}+\bar{\Lambda} \otimes \bar{\Gamma}+\bar{\Gamma} \otimes \bar{\Lambda}+\bar{\Lambda} \otimes \bar{\Gamma} \otimes \bar{\Lambda}+\cdots
$$

of all words formed by tensoring $\bar{\Lambda}$ and $\bar{\Gamma}$ together with no repetitions allowed. There is an evident $K$-linear map

$$
W \longrightarrow \Lambda * \Gamma
$$

given on the fifth summand above, for example, by

$$
\bar{\Lambda} \otimes \bar{\Gamma} \otimes \bar{\Lambda} \longrightarrow \Lambda \otimes \Gamma \otimes \Lambda \longrightarrow(\Lambda \oplus \Gamma) F \longrightarrow \Lambda * \Gamma
$$

The map $W \longrightarrow \Lambda * \Gamma$ is one-one because its image in $F$ does not intersect the ideal $I$ $((\Lambda+\Gamma) F / I=\Lambda * \Gamma)$ and it is onto, clearly. Thus viewing $\Lambda * \Gamma$ as a $K$-module, we have

$$
\Lambda * \Gamma=K+\bar{\Lambda}+\bar{\Gamma}+\bar{\Lambda} \otimes \bar{\Gamma}+\cdots
$$

Now let $\Lambda \stackrel{f}{\longrightarrow} \Lambda_{1}, \Gamma \stackrel{g}{\longrightarrow} \Gamma_{1}$, be $K$-linear maps which respect both units and supplementations, that is, writing $\Lambda=K \oplus \bar{\Lambda}$, and $\Lambda_{1}, \Gamma, \Gamma_{1}$ similarly,

$$
\begin{array}{ll}
K \oplus \bar{\Lambda} \quad \stackrel{f=K \oplus \bar{f}}{\longrightarrow} K \oplus \bar{\Lambda}_{1} \\
K \oplus \bar{\Gamma} \stackrel{g=K \oplus \bar{g}}{\longrightarrow} \quad K \oplus \bar{\Gamma}_{1}
\end{array}
$$


Then a $K$-linear map $\Lambda * \Gamma \longrightarrow \Lambda_{1} * \Gamma_{1}$, which we take the liberty of denoting by $f * g$, is induced:

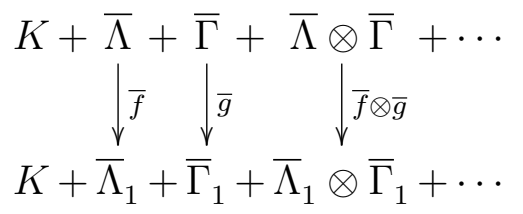

If we are also given $f_{1}: \Lambda_{1} \longrightarrow \Lambda_{2}, g_{1}: \Gamma_{1} \longrightarrow \Gamma_{2}$, then $f_{1} * g_{1}: \Lambda_{1} * \Gamma_{1} \longrightarrow \Lambda_{2} * \Gamma_{2}$ and functoriality holds: $(f * g)\left(f_{1} * g_{1}\right)=\left(f f_{1} * g g_{1}\right)$ (because $\overline{f f_{1}}=\overline{f f_{1}}$, and similarly for $g$, $\left.g_{1}\right)$.

To complete the argument, let

$$
\Lambda \stackrel{K \oplus \bar{h}_{-1}}{\longrightarrow} \Lambda G \stackrel{K \oplus \bar{h}_{0}}{\longrightarrow} \Lambda G^{2} \longrightarrow \cdots \longrightarrow \Lambda G^{n+1} \stackrel{K \oplus \bar{h}_{n}}{\longrightarrow} \cdots
$$

be the $K$-contraction with diagonal matrix constructed above. The $K \oplus h_{n}$ preserve both units and supplementations, so

$$
\Lambda * \Gamma \stackrel{\left(K \oplus \bar{h}_{-1}\right) * \Gamma}{\longrightarrow} \Lambda G * \Gamma \longrightarrow \cdots \longrightarrow \Lambda G^{n+1} * \Gamma \stackrel{\left(K \oplus \bar{h}_{n}\right) * \Gamma}{\longrightarrow} \cdots
$$

is a sequence of well defined $K$-linear maps which satisfies the contraction identities by virtue of the above functoriality.

Alternative ARgument. The commutative diagram of adjoint pairs

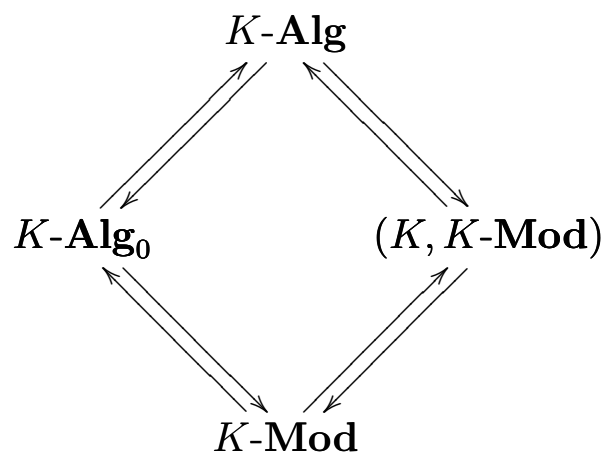

arises from a distributive law $T S \longrightarrow S T$ in $K$-Mod; $T$ is the triple $M \longrightarrow K \oplus M$, whose algebras are unitary $K$-modules (objects of the comma category $(K, K$-Mod)) and $S$ is the triple $M \longrightarrow M+M \otimes M+\cdots$, whose algebras are associative $K$-algebras without unit; this is the category denoted $K \mathbf{A l g}_{0}$. Let $\mathbf{G}$ be the cotriple in $K$-Alg relative to $K$-Mod, and $\mathbf{G}_{1}$ that relative to unitary $K$-modules $(K, K$-Mod $)$. By an easy extrapolation of [Barr (1969), 5.2], the cotriples $\mathbf{G}, \mathbf{G}_{1}$ operate in the full subcategory $K$-Alg' ${ }^{\prime}$ consisting of those $\Lambda \in K$-Alg whose underlying unitary $K$-modules are projective relative to $K$ modules, and $\mathbf{G}, \mathbf{G}_{1}$ restricted to this subcategory have the same projective objects. Now $\Lambda$, as a unitary $K$-module, is projective relative to $K$-modules $\Leftrightarrow$ there is a commutative 
diagram of $K$-linear maps

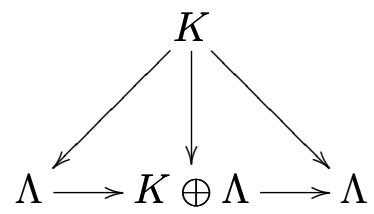

$\Leftrightarrow \Lambda$ has a $K$-linear supplementation. Thus if $\Lambda$ is such an algebra, the standard resolutions

$$
\left(\Lambda G^{n+1}\right),\left(\Lambda G_{1}^{n+1}\right)_{n \geq-1}
$$

are chain equivalent in $\mathbf{Z}(K-\mathbf{A l g})$. ( ) $* \Gamma$ extends to an additive endofunctor of $\mathbf{Z}(K-\mathbf{A l g})$.

$$
\left(\Lambda G^{n+1} * \Gamma\right),\left(\Lambda G_{1}^{n+1} * \Gamma\right)_{n \geq-1}
$$

are therefore also chain equivalent in $\mathbf{Z}\left(K\right.$-Alg). Finally, $\left(\Lambda G_{1}^{n+1} * \Gamma\right)_{n \geq-1}$ has a $K$-linear contraction. This implies that

$$
H_{p}\left(\Lambda G,\left(\Lambda G^{n+1} * \Gamma\right)_{n \geq-1}\right)=0, \quad p \geq 0,
$$

so $\left(\Lambda G^{n+1} * \Gamma\right)$ is $\mathbf{G}$-acyclic.

As to the last $K$-contraction, if $\Lambda$ is any $K$-algebra for a moment, and $\Gamma$ is $K$-linearly supplemented, then as a $K$-module $\Lambda * \Gamma$ can be viewed as a direct sum

$$
\Lambda+\Gamma+\Lambda \otimes \bar{\Gamma}+\bar{\Gamma} \otimes \Lambda+\cdots
$$

modulo the relations $\gamma \otimes 1_{\Lambda}=1_{\Lambda} \otimes \gamma=\gamma$, and the ideal generated by them, such as $\lambda \otimes \gamma \otimes 1_{\Lambda}=\lambda \otimes \gamma, \ldots$ Thus if $f: \Lambda \longrightarrow \Lambda_{1}$ is a unitary $K$-linear map, " $f * \Gamma$ ": $\Lambda * \Gamma \longrightarrow \Lambda_{1} * \Gamma$ is induced, and functoriality holds: $f f_{1} * \Gamma=(f * \Gamma)\left(f_{1} * \Gamma\right)$. Now the resolution $\left(\Lambda G_{1}^{n+1}\right)_{n \geq-1}$ has a unitary $K$-linear contraction (5.3). This contraction goes over into $\left(\Lambda G_{1}^{n+1} * \Gamma\right)_{n \geq-1}$ provided $\Gamma$ is $K$-linearly supplemented.

\section{Homology coproduct theorems}

Let $\mathbf{G}$ be a cotriple in $\mathbf{C}$ and let $E$ be a coefficient functor $\mathbf{C} \longrightarrow \mathscr{A}$. E preserves coproducts if the map induced by the coproduct injections $X, Y \rightarrow X * Y$ is an isomorphism for all $X, Y \in \mathbf{C}$ :

$$
X E \oplus Y E \stackrel{\cong}{\longrightarrow}(X * Y) E
$$

(In this section we assume $\mathbf{C}$ has coproducts). Particularly if $E$ preserves coproducts, it is plausible that the similarly-defined natural map in homology is an isomorphism; if it is indeed the case that

$$
H_{n}(X, E)_{\mathbf{G}} \oplus H_{n}(Y, E)_{\mathbf{G}} \longrightarrow H_{n}(X * Y, E)_{\mathbf{G}}
$$

is an isomorphism, we say that the homology coproduct theorem holds (strictly speaking, for the objects $X, Y$, in dimension $n$; it is characteristic of the theory to be developed that the coproduct theorem often holds only for objects $X, Y$ with special properties). 
In this section we show that the homology coproduct theorem holds for the various categories and various cotriples considered in Section 6. As one gathers from the arguments resorted to in that section, there must be something the matter with the slick method of proving coproduct theorems sketched in [Barr \& Beck (1966), §5]. First, to correct a slip, (5.4) in [Barr \& Beck (1966)] should read $u:\left(X_{1} * X_{2}\right) G U \longrightarrow\left(X_{1} G * X_{2} G\right) U$, that is, it is in the underlying category that $u$ should be sought. However, even with that correction, such a natural $u$ does not exist so far as we know, in group theory (relative to sets) or in Hochschild theory, contrary to our earlier claims. The morphisms $u$ which we had in mind in these cases turned out on closer inspection not to be natural, because of misbehavior of neutral elements of one kind or another in coproducts viewed at the underlying-category level. Only in "case 3" of [Barr \& Beck (1966), §5], namely that of commutative algebras relative to $K$-modules, does the method of that paper work. However, we are able to retrieve most of the results claimed there although in the case of Hochschild theory we are forced to impose an additional linear-supplementation hypothesis.

Such tests as we possess for the coproduct theorem are contained in the next two propositions.

Proposition (7.1). If $X$ and $Y$ possess $\mathbf{G}$-resolutions

$$
\begin{gathered}
X=X_{-1} \longleftarrow X_{0} \leftleftarrows X_{1} \leftleftarrows \\
Y=Y_{-1} \longleftarrow Y_{0} \leftleftarrows \\
\longleftarrow
\end{gathered}
$$

such that the coproduct

$$
X * Y \longleftarrow X_{0} * Y_{0} \leftleftarrows X_{1} * Y_{1} \leftleftarrows
$$

is a $\mathbf{G}$-resolution, then the coproduct theorem holds for $X, Y$ and any coproduct-preserving coefficient functor. (The issue is $\mathbf{G}$-acyclicity.)

In particular, if each row and column of the double augmented simplicial object

$$
\left(X_{m} * Y_{n}\right)_{m, n \geq-1}
$$

is a $\mathbf{G}$-resolution, then the above diagonal object $\left(X_{n} * Y_{n}\right)_{n \geq-1}$ is a $\mathbf{G}$-resolution.

Proposition (7.2). Suppose that the cotriple $\mathbf{G}$ factors through an adjoint pair

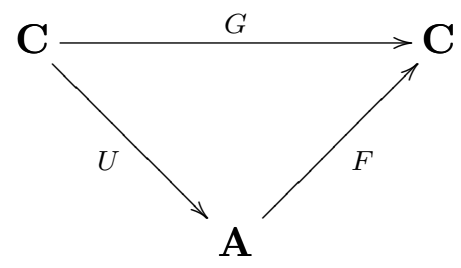

and that $(X * Y) U$ is naturally equivalent to $X U *_{\mathbf{A}} Y U$ where $*_{\mathbf{A}}: \mathbf{A} \times \mathbf{A} \longrightarrow \mathbf{A}$ is some bifunctor; in other words, the coproduct is definable at the underlying-category level. Then the homology coproduct theorem holds for any coproduct-preserving coefficient functor. 
As to (7.1), coproducts of projectives being projective, we are left to consider the augmented double simplicial set

$$
\left(A G, X_{m} * Y_{n}\right)_{m, n \geq-1}
$$

As the rows and columns lack homology, so does the diagonal, by the Eilenberg-Zilber theorem [Eilenberg \& Zilber (1953)]. For (7.2), identify

$$
\left(\left(X G^{n+1} * Y G^{n+1}\right) U\right)_{n \geq-1} \text { with }\left(X G^{n+1} U *_{\mathbf{A}} Y G^{n+1} U\right)
$$

of which

$$
X U *_{\mathbf{A}} \stackrel{h_{-1} *_{\mathbf{A}} k_{-1}}{\longrightarrow} X G U *_{\mathbf{A}} Y G U \longrightarrow \cdots
$$

is a contraction (see (5.3)).

In the following examples we use the fact that the coproduct in the category of objects over $X,(\mathbf{C}, X)$, is "the same" as the coproduct in $\mathbf{C}$ :

$$
\left(X_{1} \stackrel{P_{1}}{\longrightarrow} X\right) *\left(X_{2} \stackrel{P_{1}}{\longrightarrow} X\right)=\left(X_{1} * X_{2} \stackrel{\left(P_{1}, P_{2}\right)}{\longrightarrow} X\right)
$$

\subsection{Groups.}

$$
H_{n}\left(\Pi_{1}, E\right) \oplus H_{n}\left(\Pi_{2}, E\right) \stackrel{\sim}{\longrightarrow} H_{n}\left(\Pi_{1} * \Pi_{2}, E\right)
$$

for any coproduct-preserving functor $E: G \longrightarrow \mathscr{A}$, such as $\otimes M$ or $\operatorname{Hom}(, M)$ where $M$ is a fixed abelian group.

To deduce the usual coproduct theorems for homology and cohomology with coefficients in a module, we apply the complement to (5.3) to see that $\left(\Pi_{1} G^{n+1}\right)_{n \geq-1}$ is a (G, $\Pi_{1} * \Pi_{2}$ )-resolution of $\Pi_{1}$ as a group over $\Pi_{1} * \Pi_{2}$ (using the coproduct injection $\left.\Pi_{1} \longrightarrow \Pi_{1} * \Pi_{2}\right)$. By (6.1) and the complement to (5.3) again, $\left(\Pi_{1} G^{n+1} * \Pi_{2} G^{n+1}\right)_{n>-1}$ is a $\left(\mathbf{G}, \Pi_{1} * \Pi_{2}\right)$-resolution of $\Pi_{1} * \Pi_{2} \rightarrow \Pi_{1} * \Pi_{2}$. If $M$ is a $\Pi_{1} * \Pi_{2}$-module, then $M$ can be regarded both as a $\Pi_{1}$-module and as a $\Pi_{2}$-module by means of $\Pi_{1}, \Pi_{2} \rightarrow \Pi_{1} * \Pi_{2}$. Thus in homology we have a chain equivalence between the complexes

$$
\begin{array}{ll}
\left(\operatorname{Diff}_{\Pi_{1} * \Pi_{2}}\left(\Pi_{1} G^{n+1}\right) \otimes M \oplus \operatorname{Diff}_{\Pi_{1} * \Pi_{2}}\left(\Pi_{2} G^{n+1}\right) \otimes M\right), & \\
\left(\operatorname{Diff}_{\Pi_{1} * \Pi_{2}}\left(\Pi_{1} G^{n+1} * \Pi_{2} G^{n+1}\right) \otimes M\right), & n \geq 0,
\end{array}
$$

$\otimes$ being over $\Pi_{1} * \Pi_{2}$. As a result,

$$
H_{n}\left(\Pi_{1}, M\right) \oplus H_{n}\left(\Pi_{2}, M\right) \stackrel{s}{\longrightarrow} i m H_{n}\left(\Pi_{1} * \Pi_{2}, M\right) .
$$

In cohomology, taking coefficients in $\operatorname{Hom}_{\Pi_{1} * \Pi_{2}}(, M)$,

$$
H^{n}\left(\Pi_{1} * \Pi_{2}, M\right) \stackrel{\sim}{\longrightarrow} H^{n}\left(\Pi_{1}, M\right) \oplus H^{n}\left(\Pi_{2}, M\right) .
$$

These isomorphisms, apparently known for some time, appear to have been first proved (correctly) in print in [Barr \& Rinehart (1966)]. Similar isomorphisms hold for (co-)homology of $W_{1} * W_{2}$ where $W_{1} \longrightarrow \Pi_{1}, W_{2} \longrightarrow \Pi_{2}$ are groups over $\Pi_{1}, \Pi_{2}$. (Earlier proof: [Trotter (1962)].) 
7.4. Commutative Algebras. Let $B, C$ be $A$-algebras over $D$, that is, $B, C \longrightarrow D$, and $M$ a $D$-module.

If $H$ is homology relative to the "absolute" cotriple $\mathbf{G}$ coming from $(A, \mathbf{C o m m}) \longrightarrow$ Sets, we have

$$
\begin{aligned}
& H_{n}(B, M) \oplus H_{n}(C, M) \stackrel{\sim}{\longrightarrow} H_{n}\left(B \otimes_{A} C, M\right), \\
& H^{n}\left(B \otimes_{A} C, M\right) \stackrel{\sim}{\longrightarrow} H^{n}(B, M) \oplus H^{n}(C, M)
\end{aligned}
$$

provided $\operatorname{Tor}_{p}^{A}(B, C)=0$ for $p>0$; this is because the coproduct of the standard resolutions,

$$
\left(B G^{n+1} \otimes_{A} C G^{n+1}\right)_{n \geq-1},
$$

has $\operatorname{Tor}(B, C)$ as its homology (use the Eilenberg-Zilber theorem), which is the obstruction to a contraction in the underlying category of sets. The result is also proved in [André (1967), Quillen (1967), Lichtenbaum \& Schlessinger (1967), Harrison (1962)].

If $H$ is the theory relative to $A$-modules, the isomorphisms hold without any condition (6.2).

7.5. Associative $K$-Algebras. If $\Lambda_{1}, \Lambda_{2} \rightarrow \Gamma$ are $K$-algebra maps and $M$ is a two-sided $\Gamma$-module, and $\Lambda_{1}, \Lambda_{2}$ possess $K$-linear supplementations, then

$$
H_{n}\left(\Lambda_{1}, M\right) \oplus H_{n}\left(\Lambda_{2}, M\right) \stackrel{\sim}{\longrightarrow} H_{n}\left(\Lambda_{1} * \Lambda_{2}, M\right),
$$

$n \geq 0$; the same cohomology with coefficients in $M$, or for any coproduct-preserving coefficient functor. The cotriple employed is that relative to $K$-modules; the proofs are from $(6.3),(7.1)$.

\section{On the homology of a map}

In Section 2 we defined homology groups of a map so as to obtain an exact sequence

$$
\cdots \longrightarrow H_{n}(X, E) \longrightarrow H_{n}(Y, E) \longrightarrow H_{n}(X \longrightarrow Y, E) \stackrel{\partial}{\longrightarrow} H_{n-1}(X, E) \longrightarrow \cdots
$$

In fact, although we had to use a mapping cone instead of a quotient complex, the definition is the same as in algebraic topology. In this section we show (with a proviso) that these groups are the same as the cotriple groups

$$
H_{n}(X \rightarrow Y,(X, E))_{(X, \mathbf{G})}, \quad n \geq 0,
$$

where $X \longrightarrow Y$ is considered as an object under $X,(X, E)$ is the extension to a functor $(X, \mathbf{C}) \longrightarrow \mathscr{A}$ of a given coefficient functor $E: \mathbf{C} \longrightarrow \mathscr{A}$ and $(X, \mathbf{G})$ is $\mathbf{G}$ lifted into the comma category as described before (1.2); the proviso is that a homology coproduct theorem should hold for the coproduct of any object with a free object.

The coefficient functor we use,

$$
(X, \mathbf{C}) \stackrel{(X, E)}{\longrightarrow} \mathscr{A}
$$


is defined by $(X \longrightarrow Y)(X, E)=$ coker $X E \longrightarrow Y E$. Recalling the formulas for $(X, \mathbf{G})$, we have that the $H_{n}(X \longrightarrow Y,(X, E))_{X, \mathbf{G}}$ are the homology groups of the standard complex which in dimensions 0 and 1 reads:

$$
0 \leftarrow \operatorname{coker}(X E \longrightarrow(X * Y G) E) \stackrel{\partial_{1}}{\longleftarrow} \operatorname{coker}(X E \longrightarrow(X *(X * Y G) G) E) \stackrel{\partial_{2}}{\longleftarrow} \cdots .
$$

THEOREM (8.1). There is a sequence of homology maps

$$
H_{n}(X \longrightarrow Y, E)_{\mathbf{G}} \stackrel{H_{n}(\varphi)}{\longrightarrow} H_{n}(X \longrightarrow Y,(X, E))_{(X, \mathbf{G})}, \quad n \geq 0,
$$

resulting from a natural chain transformation

$$
C(X \longrightarrow Y)_{n} \stackrel{\varphi_{n}}{\longrightarrow}(X \longrightarrow Y)(X, \mathbf{G})^{n+1}(X, E) .
$$

$(C(X \longrightarrow Y)$ is the mapping defined in Section 2 and functoriality is respect to maps of objects under $X)$. The $H_{n}(\varphi)$ are isomorphisms if the following theorem holds: for all $X, Y \in \mathbf{C}$, the coproduct injections induce isomorphisms

$$
H_{n}(X * Y G, E)_{\mathbf{G}} \stackrel{\sim}{\simeq} \begin{cases}H_{0}(X, E)_{\mathbf{G}} \oplus Y G E, & n=0, \\ H_{n}(X, E)_{\mathbf{G}}, & n>0,\end{cases}
$$

that is, if E satisfies the homology coproduct theorem when one summand is $\mathbf{G}$-free.

ProOF. We augment both complexes by attaching $H_{0}$ as $(-1)$-dimensional term. We first define $\varphi_{0}, \varphi_{1}$ so as to obtain the commutative square $\varphi_{1} \partial_{1}=\partial_{1} \varphi_{0}$, which induces a natural map $\varphi_{-1}$ on the augmentation terms.

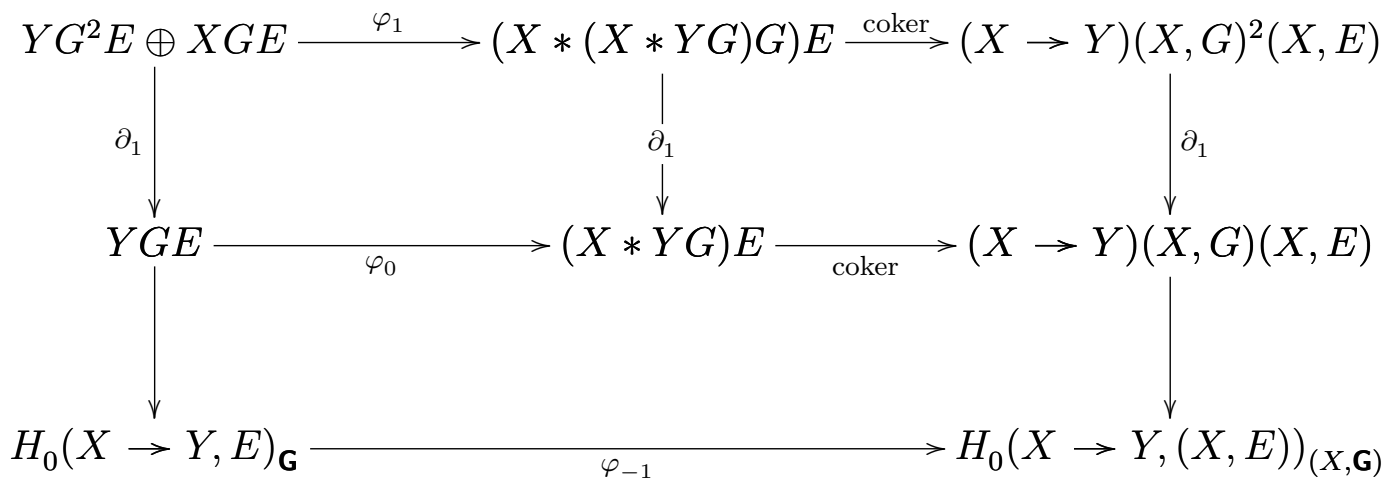

If we write $i: X \longrightarrow Y * Y G, j: Y G \longrightarrow X * Y G$ for coproduct injections, then $\varphi_{0}=j E$, and $\varphi_{1}$ is determined by

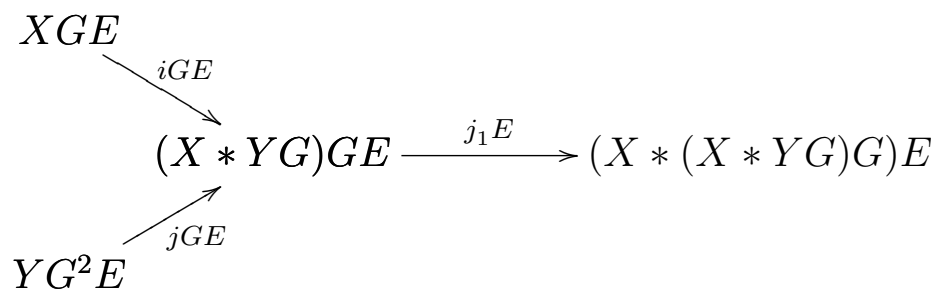


where $j_{1}$ is also a coproduct injection. That $\varphi_{0}, \varphi_{1}$ commute with $\partial_{1}$ is readily checked.

The higher $\varphi_{n}$ could be written down similarly but we don't bother with that as they automatically fall out of the acyclic-models argument which we need for the isomorphism anyway. We use $(X, \mathbf{G})$ as the comparison cotriple. The cotriple complex $(X \longrightarrow Y)(X, G)^{n+1}(X, E)$ is representable and contractible with respect to this cotriple, as always. Furthermore, $C(X \longrightarrow Y)$ is $(X, \mathbf{G})$-representable via

$$
\begin{gathered}
\vartheta_{n}: C(X \longrightarrow Y)_{n} \longrightarrow C(X \longrightarrow X * Y G)_{n} \\
Y G^{n+1} E \oplus X G^{n} E \stackrel{(Y \delta . j G) G^{n} E \oplus \text { id. }}{\longrightarrow}(X * Y G) G^{n+1} E \oplus X G^{n} E,
\end{gathered}
$$

if $n>0$, and $\vartheta_{0}=j E$. This proves $\varphi_{-1}$ can be extended to a chain transformation defined in all dimensions. It happens that the extension produced by (11.1) agrees with the above $\varphi_{0}, \varphi_{1}$ in the lowest dimensions.

To conclude, if the homology coproduct assumption in (8.1) holds, then

$$
H_{n}(X \rightarrow X * Y G, E)_{\mathbf{G}} \simeq \begin{cases}Y G E, & n=0, \\ 0, & n>0,\end{cases}
$$

since this homology group $H_{n}$ fits into the exact sequence

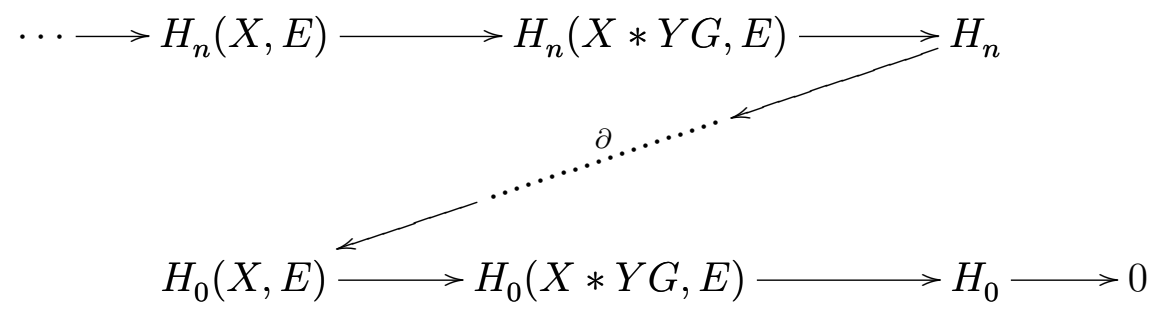

Thus the $\varphi_{n}$ induce homology isomorphisms between the two theories (11.3).

\subsection{Groups. If}

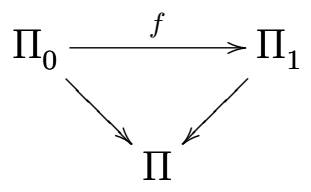

is a map in $(\mathscr{G}, \Pi)$ and $M$ is a $\Pi$-module we get an exact sequence

$$
\cdots \longrightarrow H_{n}\left(\Pi_{0}, M\right) \longrightarrow H_{n}\left(\Pi_{1}, M\right) \longrightarrow H_{n}(f, M) \longrightarrow H_{n-1}\left(\Pi_{0}, M\right) \longrightarrow \cdots
$$

and a similar one in cohomology. The relative term arises either as in Section 2 or by viewing $f$ as an object in the double comma category $\left(\Pi_{0}, \mathscr{G}, \Pi_{1}\right)$ and using this section. The equivalence results from the fact that the homology coproduct theorem holds for groups.

This sequence can be obtained topologically by considering the map of Eilenberg-Mac Lane spaces $K\left(\Pi_{0}, 1\right) \longrightarrow K\left(\Pi_{1}, 1\right)$. It is also obtained in [Takasu $(1959 / 60)$ ]. 
As a special case, if

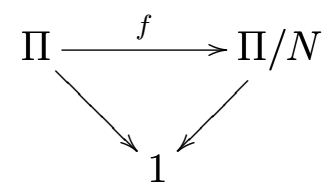

is division by a normal subgroup and we take coefficients in $\mathbf{Z}$ as a 1-module, then $H_{0}(f)=$ 0 and $H_{1}(f) \simeq N /[\Pi, N]$. Thus the Stallings-Stammbach sequence ([Stallings (1965), Stammbach (1966)]) falls out:

$$
H_{1}(\Pi) \longrightarrow H_{1}(\Pi / N) \longrightarrow N /[\Pi, N] \longrightarrow H_{0}(\Pi) \longrightarrow H_{0}(\Pi / N) \longrightarrow 0
$$

(our dimensional indices). Doubtless many of the other sequences of this type given in [Eckmann \& Stammbach (1967)] can be got similarly.

8.3. Commutative Rings And algebras. Given maps of commutative rings

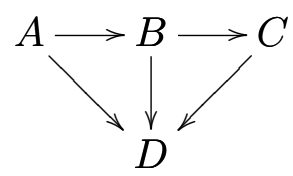

we obtain exact sequences

$$
\begin{gathered}
\cdots \longrightarrow H_{n}(A, M) \longrightarrow H_{n}(B, M) \longrightarrow H_{n}(A \longrightarrow B, M) \stackrel{\partial}{\longrightarrow} H_{n-1}(A, M) \longrightarrow \cdots \\
\cdots \longrightarrow H_{n}(A \longrightarrow B, M) \longrightarrow H_{n}(A \longrightarrow C, M) \longrightarrow H_{n}(B \longrightarrow C, M) \stackrel{\partial}{\longrightarrow} H_{n-1}(A \longrightarrow B, M) \longrightarrow \cdots
\end{gathered}
$$

for a $D$-module $M$; similar sequences are obtainable in cohomology. Taking $B=C=D$, and homology with respect to the cotriple $\mathbf{G}$ arising from

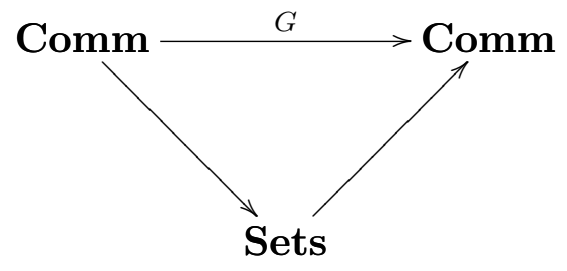

these sequences coincide with those of [Lichtenbaum \& Schlessinger (1967), André (1967), Quillen (1967)], as a result of the following facts:

(a) $(A, \mathbf{G})$ is the cotriple arising from

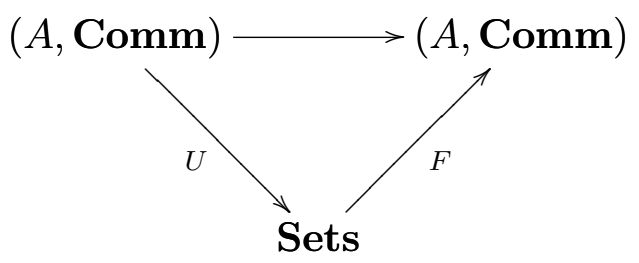

where $(A \longrightarrow B) U=B$. 
(b) If $E$ : $(\mathbf{C o m m}, D) \longrightarrow D$-Mod is $A E=\operatorname{Diff}_{D}(A) \otimes_{D} M$, then $(A \longrightarrow B)(A, E)=$ $\Omega_{B / A}^{1} \otimes_{B} M$. If $E:(\mathbf{C o m m}, D)^{*} \longrightarrow D$-Mod is $A E=\operatorname{Der}(A, M)_{D}$, then

$$
(A \longrightarrow B)(A, E)=A-\operatorname{Der}(B, M)_{D}
$$

(c)

$$
H_{n}(A \longrightarrow B, E)_{\mathbf{G}} \stackrel{\sim}{\longrightarrow} H_{n}(A \longrightarrow B,(A, E))_{A, \mathbf{G}}
$$

for any coproduct preserving coefficient functor $E:(\mathbf{C o m m}, D) \rightarrow \mathscr{A}$ (writing $A$ for $A \longrightarrow D)$.

(a) has been noted in Section 1. For (b),

$$
\begin{aligned}
(A \longrightarrow B)(A, E) & =\operatorname{coker}\left(\operatorname{Diff}_{D}(A) \otimes_{D} M \longrightarrow \operatorname{Diff}_{D}(B) \otimes_{D} M\right) \\
& =\operatorname{coker}\left(\operatorname{Diff}_{D}(A) \longrightarrow \operatorname{Diff}_{D}(B)\right) \otimes_{D} M \\
& =\left(\Omega_{B / A}^{1} \otimes_{B} D\right) \otimes_{D} M \\
& =\Omega_{B / A}^{1} \otimes_{B} M .
\end{aligned}
$$

In the dual theory, it is appropriate to lift a functor $E: \mathbf{C}^{*} \longrightarrow \mathscr{A}$ to a functor

$$
(E, A):\left(\mathbf{C}^{*}, A\right) \longrightarrow \mathscr{A}
$$

by defining $(B \longrightarrow A)(E, A)=\operatorname{ker}(B E \longrightarrow A E)$. For $E$ the contravariant functor in $(\mathrm{b})$, we have then

$$
\begin{aligned}
(A \longrightarrow B)(A, E) & =\operatorname{ker}\left(\operatorname{Der}(B, M)_{D} \longrightarrow \operatorname{Der}(A, M)\right) \\
& =A-\operatorname{Der}(B, M)_{D} .
\end{aligned}
$$

Alternatively and of course equivalently, dualize the coefficient category of $D$-modules. Finally (c) follows from the fact that the coproduct theorem holds for homology in this category when one factor is free. Indeed, $C .(A, G)$ is the polynomial $A$-algebra $A[C]$ and is $A$-flat; thus the coproduct ()$\otimes_{A} C .(A, G)$ preserves $(A, \mathbf{G})$-resolutions.

For the $A$-relative theory (1.2), the same exact sequences are available.

8.4. Associative Algebras. Let $\mathbf{G}$ denote the cotriple on $K$-Alg arising out of the adjoint pair

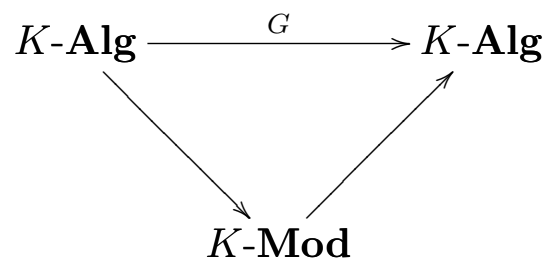

If $\Lambda \in K$-Alg, $\Gamma \longrightarrow \Lambda \in(K$-Alg, $\Lambda)$ and $M$ is a $\Lambda$-bimodule, we let $H_{n}(\Gamma, M)_{\mathbf{G}}$ and $H^{n}(\Gamma, M)_{\mathbf{G}}$ denote the derived functors with respect to $\mathbf{G}$ of $\operatorname{Diff}_{\Lambda}() \otimes_{\Lambda} e M$ and $\operatorname{Der}_{\Lambda}(, M)$ respectively. Let us drop $\Lambda$ from the notation from now on. Hence $\Gamma \longrightarrow \Gamma_{1}$ below really refers to $\Gamma \longrightarrow \Gamma_{1} \longrightarrow \Lambda$ etc. 
THEOREM (8.5).

$$
\begin{aligned}
& H_{n}\left(\Gamma \longrightarrow \Gamma_{1}, M\right)_{\mathbf{G}} \simeq H_{n}\left(\Gamma_{1}, M\right)_{(\Gamma, \mathbf{G})}, \\
& H^{n}\left(\Gamma \longrightarrow \Gamma_{1}, M\right)_{\mathbf{G}} \simeq H^{n}\left(\Gamma_{1}, M\right)_{(\Gamma, \mathbf{G})} .
\end{aligned}
$$

Proof. According to (8.1) this requires showing that for any $\Gamma^{\prime}$,

$$
H_{n}\left(\Gamma * \Gamma^{\prime} G, M\right)_{\mathbf{G}} \simeq \begin{cases}H_{0}(\Gamma, M)_{\mathbf{G}} \oplus \operatorname{Der}\left(\Gamma^{\prime} G, M\right), & n=0 \\ H_{n}(\Gamma, M)_{\mathbf{G}}, & n>0\end{cases}
$$

and similarly for cohomology. Before doing this we require

Proposition. Let $\mathbf{G}_{1}$ be the cotriple described in (6.3) above. Then

$$
\begin{aligned}
& H_{n}(\Gamma, M)_{\mathbf{G}} \simeq H_{n}(\Gamma, M)_{\mathbf{G}_{1}}, \\
& H^{n}(\Gamma, M)_{\mathbf{G}} \simeq H^{n}(\Gamma, M)_{\mathbf{G}_{1}} .
\end{aligned}
$$

The proof will be given at the end of this section.

Now observe that any $\mathbf{G}$-projective is $\mathbf{G}_{1}$-projective and also is supplemented. Now $\left(\Gamma G_{1}^{n+1}\right)_{n \geq 0}$ is a $\mathbf{G}_{1}$-resolution of $\Gamma$, which means it has a unitary $K$-linear contraction. As observed in (6.3) above, $\left(\Gamma G_{1}^{n+1} * \Gamma^{\prime} G\right)_{n \geq 0}$ also has a unitary $K$-linear contraction and it clearly consists of $\mathbf{G}_{1}$-projectives. Thus it is a $\mathbf{G}_{1}$-resolution of $\Gamma * \Gamma^{\prime} G$. But then

$$
\operatorname{Diff}_{\Lambda}\left(\Gamma G_{1}^{n+1} * \Gamma^{\prime} G\right)_{n \geq 0} \simeq \operatorname{Diff}_{\Lambda}\left(\Gamma G_{1}^{n+1}\right)_{n \geq 0} \oplus \operatorname{Diff}_{\Lambda} \Gamma^{\prime} G
$$

the second summand being a constant simplicial object, and the result follows easily.

To prove (8.6) we use acyclic models in form (11.3) below with $\mathbf{G}_{1}$ as the comparison cotriple. First observe that there is a natural transformation $\varphi: G \longrightarrow G_{1}$ which actually induces a morphism of cotriples (meaning it commutes with both comultiplication and counit). Actually $\mathbf{G}_{1}$ is presented as a quotient of $\mathbf{G}$ and $\varphi$ is the natural projection. Now we prove the theorem for cohomology. The proof for homology is similar. For any $\Gamma^{\prime} \longrightarrow \Gamma$ and any $\Gamma$-bimodule $M$, let $\Gamma^{\prime} E=\operatorname{Der}\left(\Gamma^{\prime}, M\right), \Gamma^{\prime} E^{n}=\operatorname{Der}\left(\Gamma^{\prime} G^{n+1}, M\right)$. Let $\bar{\varphi}: \operatorname{Der}\left(\Gamma^{\prime}, M\right) \longrightarrow \Gamma^{\prime} E$ be the identity and $\bar{\varphi}^{n}: \operatorname{Der}\left(\Gamma^{\prime} G_{1}^{n+1}, M\right) \longrightarrow \Gamma^{\prime} E^{n}$ be the map $\operatorname{Der}\left(\Gamma^{\prime} \varphi^{n+1}, M\right)$. Define $\vartheta^{n}: \Gamma^{\prime} G_{1} E^{n} \longrightarrow \Gamma^{\prime} E^{n}$ to be the composite

$$
\operatorname{Der}\left(\Gamma^{\prime} G_{1} G^{n+1}, M\right) \stackrel{\operatorname{Der}\left(\Gamma^{\prime} \varphi G^{n+1}, M\right)}{\longrightarrow} \operatorname{Der}\left(\Gamma^{\prime} G^{n+2}, M\right) \stackrel{\operatorname{Der}\left(\Gamma^{\prime} \delta G^{n}, M\right)}{\longrightarrow} \operatorname{Der}\left(\Gamma^{\prime} G^{n+1}, M\right)
$$

Then it is easily seen that $\operatorname{Der}\left(\Gamma^{\prime} \varepsilon_{1} G^{n+1}, M\right) \cdot \vartheta^{n}$ is the identity. (Of course, everything is dualised for cohomology.) Thus the proof is finished by showing that the complex

$$
\cdots \longrightarrow \Gamma^{\prime} G_{1} E^{n} \longrightarrow \Gamma^{\prime} G_{1} E^{n-1} \longrightarrow \cdots \longrightarrow \Gamma^{\prime} G_{1} E^{1} \longrightarrow \Gamma^{\prime} G_{1} E \longrightarrow 0
$$

is exact. But the homology of that complex is simply the Hochschild homology of $\Gamma^{\prime} G$, (with the usual degree shift), which in turn is $\operatorname{Ext}_{\left(\Gamma^{e}, K\right)}\left(\operatorname{Diff}_{\Gamma^{\prime}} \Gamma^{\prime} G_{1}, M\right)$. Hence we complete 
the proof by showing that $\operatorname{Diff}_{\Gamma} \Gamma^{\prime} G$ is a $K$-relative $\Gamma^{e}$-projective. But $\operatorname{Der}\left(\Gamma^{\prime} G_{1}, M\right)$ consists of those derivations of $\Gamma^{\prime} G \longrightarrow M$ which vanish on the ideal of $\Gamma^{\prime} G$ generated by $1_{\Gamma^{\prime}}-1_{K}$ or, since all derivations vanish on $1_{K}$, it simply consists of those derivations which vanish on $1_{\Gamma^{\prime}}$. But $\operatorname{Der}\left(\Gamma^{\prime} G, M\right) \simeq \operatorname{Hom}_{K}\left(\Gamma^{\prime}, M\right)$ and it is easily seen that $\operatorname{Der}\left(\Gamma^{\prime} G_{1}, M\right) \simeq$ $\operatorname{Hom}_{K}\left(\Gamma^{\prime} / K, M\right)$ where $\Gamma^{\prime} / K$ denotes $\operatorname{coker}(K \longrightarrow \Gamma)$. This in turn is $\simeq \operatorname{Hom}_{\Lambda} e\left(\Lambda^{e} \otimes\right.$ $\left.\Gamma^{\prime} / K, M\right)$ and so $\operatorname{Diff}_{\Gamma} \Gamma^{\prime} G_{1} \simeq \Lambda^{e} \otimes \Gamma^{\prime} / K$ which is clearly a $K$-relative $\Lambda^{e}$-projective. This completes the proof.

\section{Mayer-Vietoris theorems}

Using assumptions about the homology of coproducts, we shall deduce some theorems of Mayer-Vietoris type. We learned of such theorems from André's work [André (1967)]. In the case of commutative algebras we obtain slightly more comprehensive results (9.5). Mostly, however, we concentrate on the case of groups (9.4).

Let $E: \mathbf{C} \longrightarrow \mathscr{A}$ be a coefficient functor.

Theorem (9.1). Let

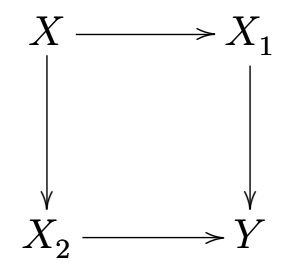

be a pushout diagram in $\mathbf{C}$ and suppose that the homology coproduct theorem holds for $Y$ viewed as a coproduct in $(X, \mathbf{C})$ :

$$
H_{n}\left(X \longrightarrow X_{1}, E\right) \oplus H_{n}\left(X \longrightarrow X_{2}, E\right) \stackrel{\sim}{\longrightarrow} H_{n}(X \longrightarrow Y, E), \quad n \geq 0 .
$$

Then there is an exact sequence,

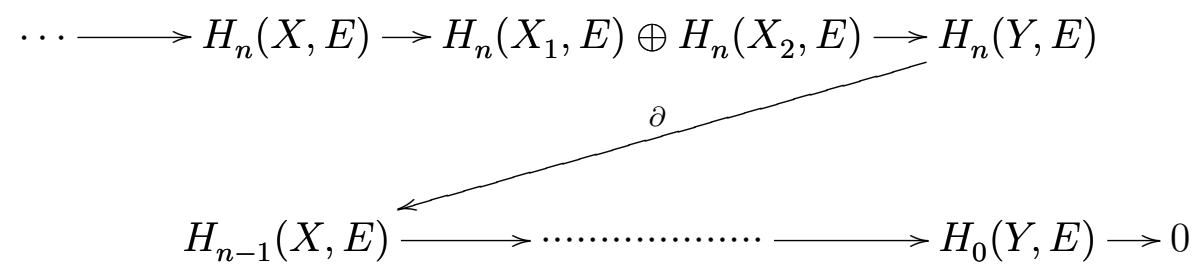

(The maps in the sequence are the usual Mayer-Vietoris maps $(\beta,-\gamma),\left(\beta_{1}, \gamma_{1}\right)$ transpose, if we momentarily write

$$
\begin{array}{ll}
\beta: H(X) \longrightarrow H\left(X_{1}\right), & \beta_{1}: H\left(X_{1}\right) \longrightarrow H(Y), \\
\gamma: H(X) \longrightarrow H\left(X_{2}\right), & \left.\gamma_{1}: H\left(X_{2}\right) \longrightarrow H(Y) .\right)
\end{array}
$$


TheOREM (9.2). Suppose that the natural map is an isomorphism

$$
H_{n}(X, E) \oplus H_{n}(Y, E) \stackrel{\sim}{\longrightarrow} H_{n}(X * Y, E), \quad n \geq 0
$$

for any $\operatorname{map} X * Y \longrightarrow Z$ there is an exact sequence

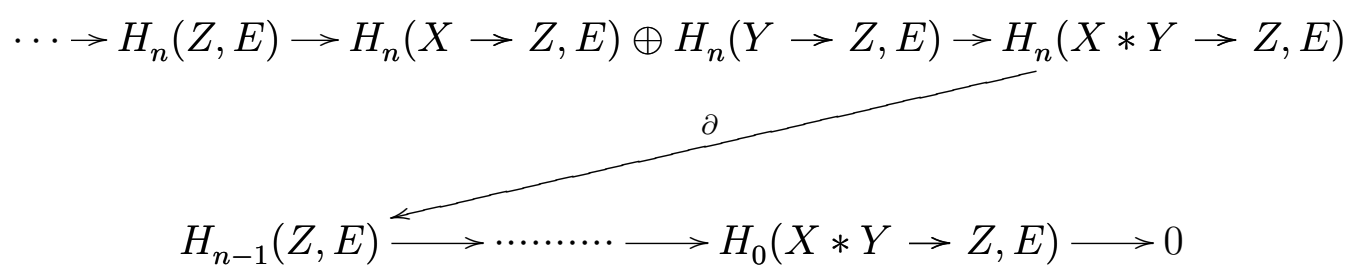

For the proof of (9.1), write down the diagram

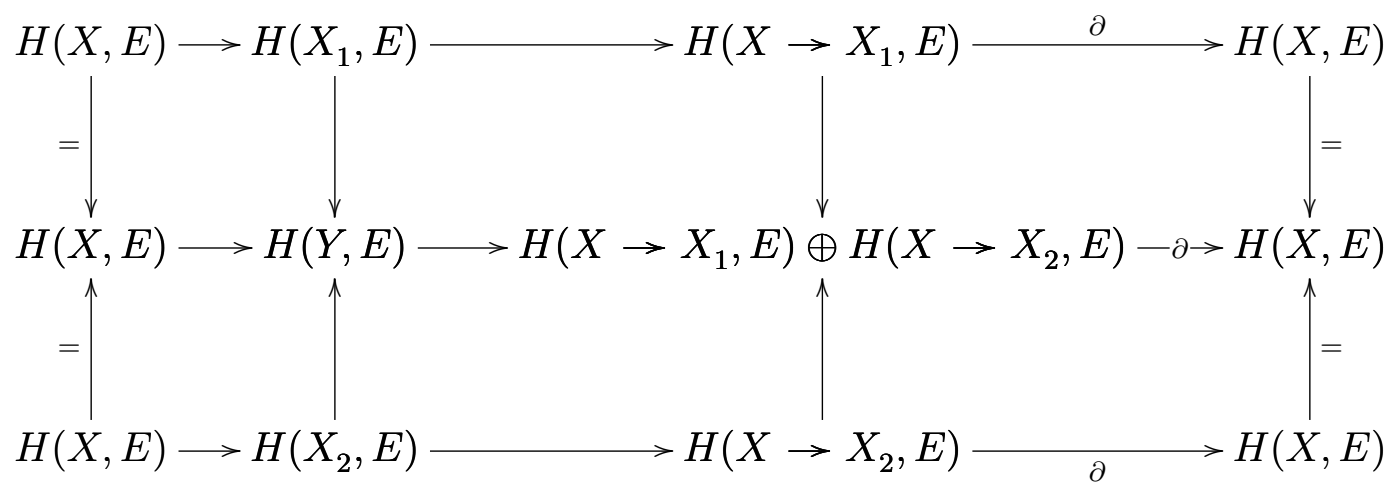

All three triangles are exact, the middle one by the coproduct theorem in $(\mathrm{X}, \mathbf{C})$. Lemma (9.3) below then yields that

$$
H(X, E) \longrightarrow H\left(X_{1}, E\right) \oplus H\left(X_{2}, E\right) \longrightarrow H(Y, E) \longrightarrow H(X, E)
$$

is an exact triangle. For (9.2), write

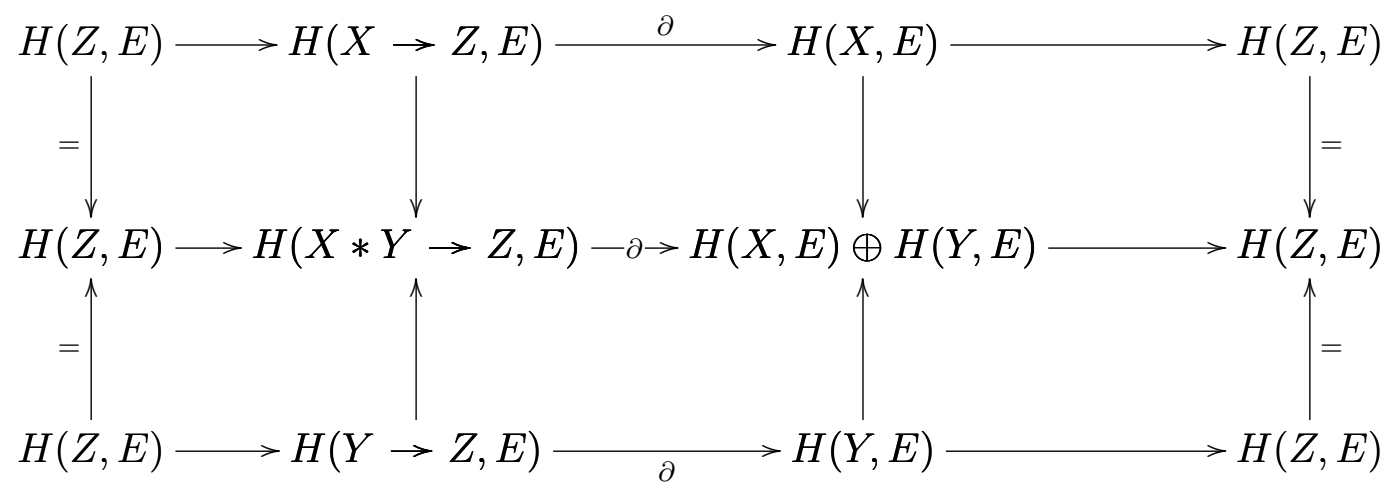

and again apply 
LEMMA (9.3). In an abelian category

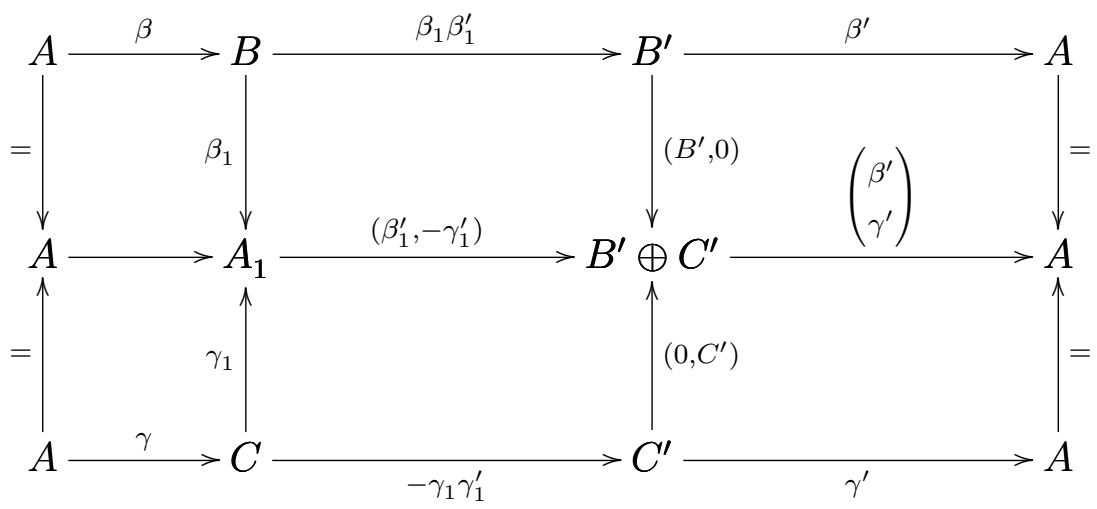

is commutative with exact triangles for rows $\Leftrightarrow$

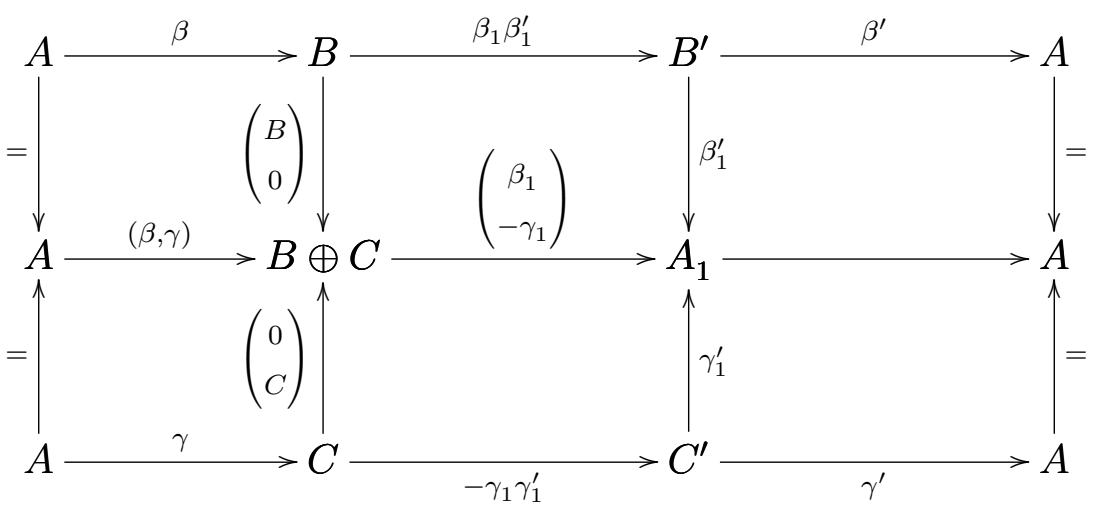

is commutative and has exact triangles for rows.

This lemma is dual to its converse and needn't be proved.

9.4 Groups. Theorem (9.2) holds without restriction. Because of the validity of the homology coproduct theorem (7.3), if $\Pi_{0} * \Pi_{1} \longrightarrow \Pi$ we get an exact sequence

$$
\begin{aligned}
\cdots \longrightarrow H_{n}(\Pi, M) \longrightarrow H_{n}\left(\Pi_{0} \longrightarrow \Pi, M\right) \oplus H_{n}\left(\Pi_{1} \longrightarrow \Pi, M\right) & \\
& \longrightarrow H_{n}\left(\Pi_{0} * \Pi_{1} \longrightarrow \Pi, M\right) \longrightarrow H_{n-1}(\Pi, M) \longrightarrow \cdots
\end{aligned}
$$

if $\mathrm{M}$ is a П-module; similar sequences hold in cohomology, or in homology with coefficients in any coproduct-preserving functor.

As to (9.1), its applicability is a little more restricted. Suppose that $\Pi_{0}$ is a subgroup of $\Pi_{1}$ and $\Pi_{2}$ and that $\Pi$ is the pushout or amalgamated coproduct:

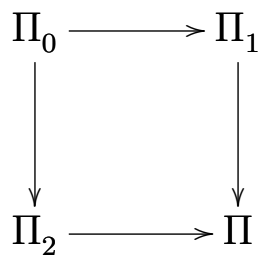


It will be shown that if $\mathrm{M}$ is a $\Pi$-module, then the $\Pi_{0}$-coproduct theorem holds for homology:

$$
H_{n}\left(\Pi_{0} \longrightarrow \Pi_{1}, M\right) \oplus H_{n}\left(\Pi_{0} \longrightarrow \Pi_{2}, M\right) \stackrel{\sim}{\longrightarrow} H_{n}\left(\Pi_{0} \longrightarrow \Pi, M\right) .
$$

Then the Mayer-Vietoris sequence

$$
\begin{aligned}
\cdots & \longrightarrow H_{n}\left(\Pi_{0}, M\right) \longrightarrow H_{n}\left(\Pi_{1}, M\right) \oplus H_{n}\left(\Pi_{2}, M\right) \longrightarrow H_{n}(\Pi, M) \\
& \longrightarrow H_{n-1}\left(\Pi_{0}, M\right) \longrightarrow H_{n-1}\left(\Pi_{1}, M\right) \oplus H_{n-1}\left(\Pi_{2}, M\right) \longrightarrow H_{n-1}(\Pi, M) \longrightarrow \cdots
\end{aligned}
$$

is exact. There is a similar exact sequence for cohomology with coefficients in $M$. While our argument will involve Diff $_{\Pi}$, we cannot claim this for arbitrary coefficient functors but only for those that are a composition of $\operatorname{Diff}_{\Pi}:(\mathscr{G}, \Pi) \longrightarrow \Pi$-Mod and an additive functor $E: \Pi-M o d \longrightarrow \mathscr{A}$. This theorem also has a topological proof using Eilenberg-Mac Lane spaces. Similar results have been obtained by [Ribes (1967)]. We now launch into the algebraic details:

The free group cotriple preserves monomorphisms: let $Y_{n}$ be the pushout or amalgamated coproduct

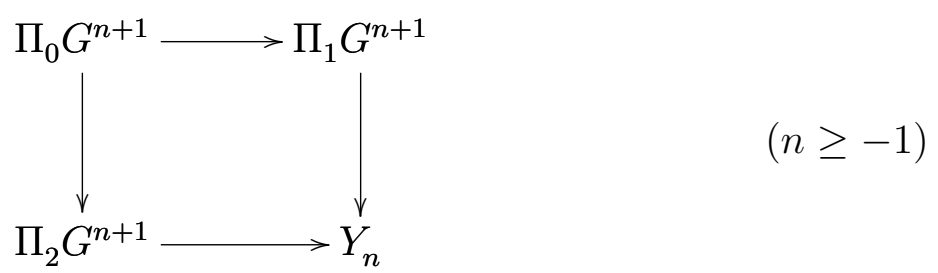

Thus $\left(Y_{n}\right)$ is an augmented simplicial group, with $Y_{-1}=\Pi$. Moreover $Y_{n}$ is a free group when $n \geq 0$ as $Y_{n}=S F$ where $S$ is the set-theoretic pushout

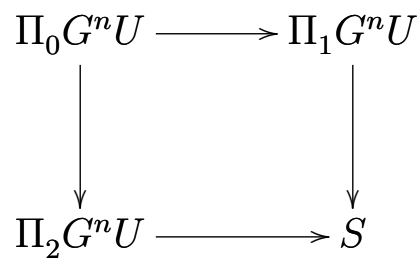

and $F$ is the free group functor $\mathscr{S} \longrightarrow \mathscr{G}$, which as a left adjoint preserves pushouts. Applying Diff $\Pi$, we get a square

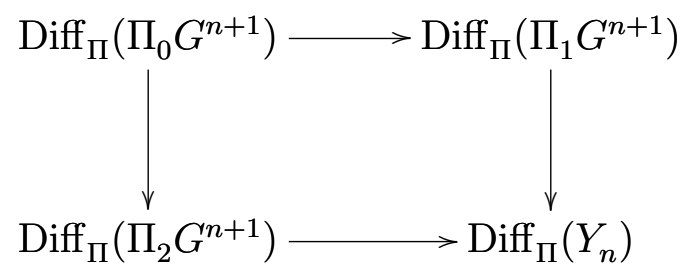

which is exact, i.e., simultaneously a pushout and a pullback. We will prove this later, as also fact (b) arrayed below. For the rest of this section we write

$$
\text { Diff }=\operatorname{Diff}_{\Pi}
$$


Now using the usual Mayer-Vietoris maps we get an exact sequence of chain complexes

$$
0 \longrightarrow\left(\operatorname{Diff}\left(\Pi_{0} G^{n+1}\right)\right) \longrightarrow\left(\operatorname{Diff}\left(\Pi_{1} G^{n+1}\right) \oplus \operatorname{Diff}\left(\Pi_{2} G^{n+1}\right)\right) \longrightarrow\left(\operatorname{Diff}\left(Y_{n}\right)\right) \longrightarrow 0
$$

for $n \geq 0$, whence the homology sequence

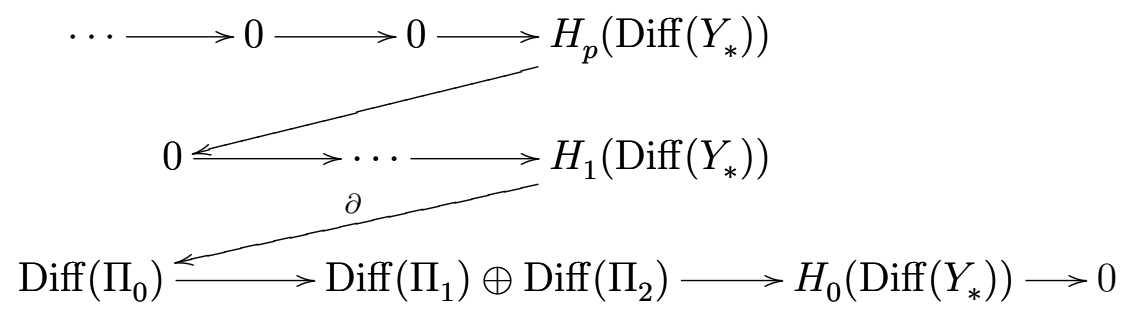

(The $p$ illustrated is $\geq 2$ ); in addition, the map $\partial$ is zero. This yields the conclusion that $\left(\operatorname{Diff}\left(Y_{n}\right)\right), n \geq-1$, is a $\Pi$-free resolution of $\operatorname{Diff}(\Pi)=I \Pi$ in the category of $\Pi$-modules.

Let $E$ be any additive functor $\Pi$-Mod $\longrightarrow \mathscr{A}$. The first two columns of the following commutative diagram are exact, hence the third column which consists of the mapping cones of the horizontal maps is also exact.

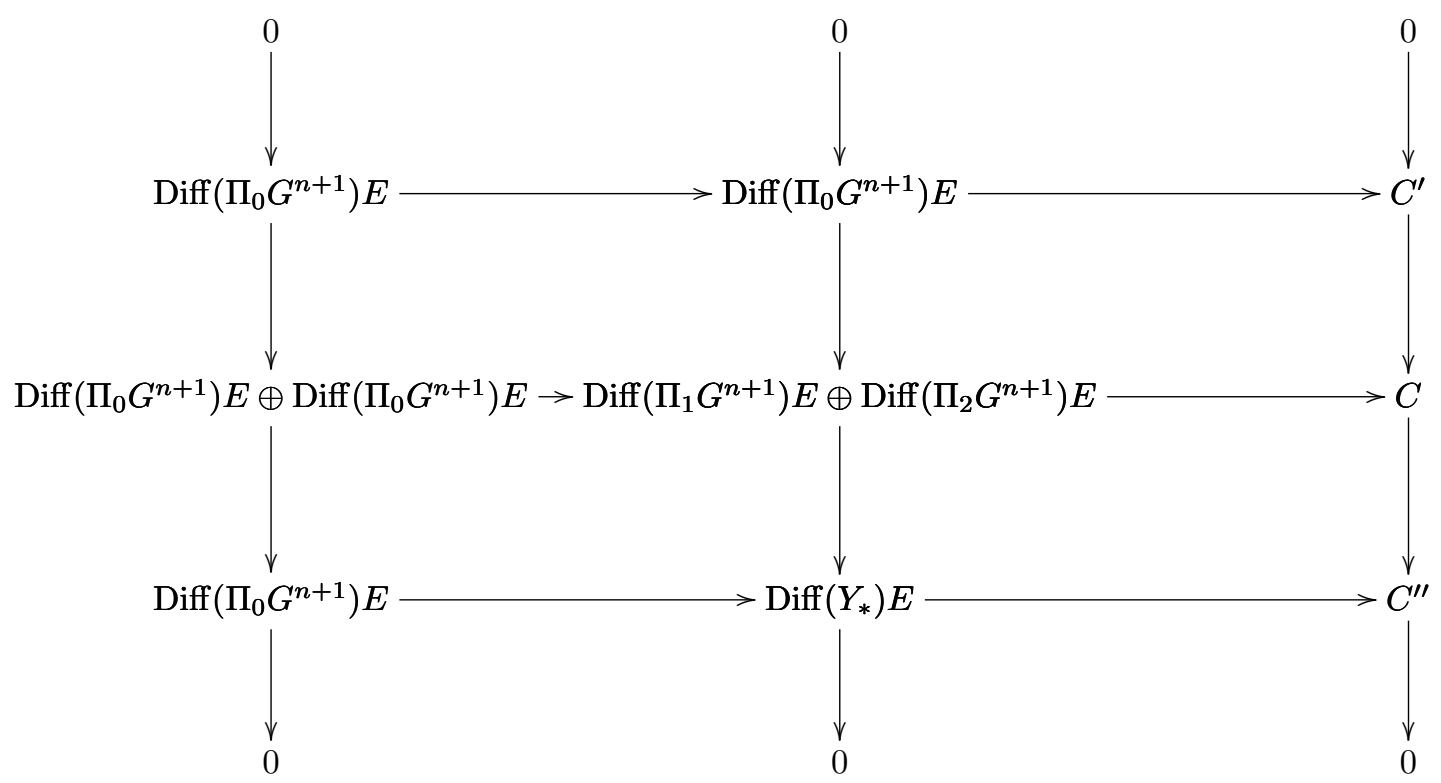

for $n \geq 0$. $C^{\prime}$ is acyclic as it is the mapping cone of an identity. Clearly,

$$
\begin{aligned}
H_{n}(C) & =H_{n}\left(\Pi_{0} \longrightarrow \Pi_{1}, E\right) \oplus H_{n}\left(\Pi_{0} \longrightarrow \Pi_{2}, E\right) \text { and } \\
H_{n}\left(C^{\prime \prime}\right) & =H_{n}\left(\Pi_{0} \longrightarrow \Pi, E\right) .
\end{aligned}
$$

The homology sequence of $0 \longrightarrow C^{\prime} \longrightarrow C \longrightarrow C^{\prime \prime} \longrightarrow 0$ then proves the coproduct theorem for groups under $\Pi_{0}$. This completes the proof that (9.1) applies to amalgamated coproduct diagrams in $\mathscr{G}$, modulo going back and proving (a), (b).

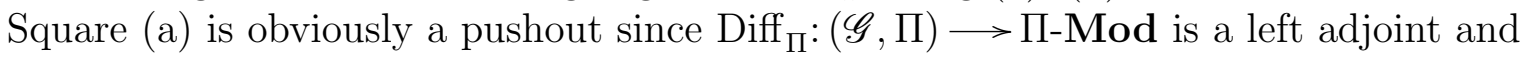
preserves pushouts. The hard part is proving that it is a pullback. For that it is enough to show that the top map $\operatorname{Diff}_{\Pi}\left(\Pi_{0} G^{n+1}\right) \longrightarrow \operatorname{Diff}_{\Pi}\left(\Pi_{1} G^{n+1}\right)$ is a monomorphism, in view of: 
LEMMA. In an abelian category,

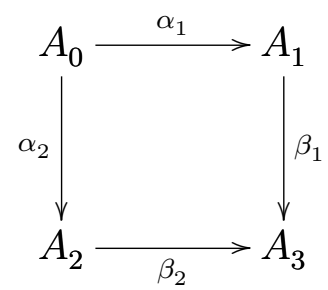

is a pushout $\Leftrightarrow$

$$
A_{0} \stackrel{\left(\alpha_{1}, \alpha_{2}\right)}{\longrightarrow} A_{1} \oplus A_{2} \stackrel{\left(\begin{array}{c}
\beta_{1} \\
-\beta_{2}
\end{array}\right)}{\longrightarrow} A_{3} \longrightarrow 0
$$

is exact, and dually, is a pullback $\Leftrightarrow$

$$
0 \longrightarrow A_{0} \stackrel{\left(\alpha_{1}, \alpha_{2}\right)}{\longrightarrow} A_{1} \oplus A_{2} \stackrel{\left(\begin{array}{c}
\beta_{1} \\
-\beta_{2}
\end{array}\right)}{\longrightarrow} A_{3}
$$

is exact.

This is standard. Thus, we are reduced to:

LEMmA. If $\Pi_{0} \longrightarrow \Pi$ is a subgroup, then $\operatorname{Diff}_{\Pi}\left(\Pi_{0}\right) \longrightarrow \operatorname{Diff}_{\Pi}(\Pi)$ is a monomorphism of I-modules. If

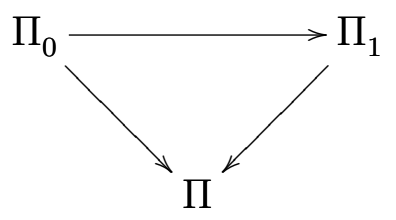

is a diagram of subgroups, then

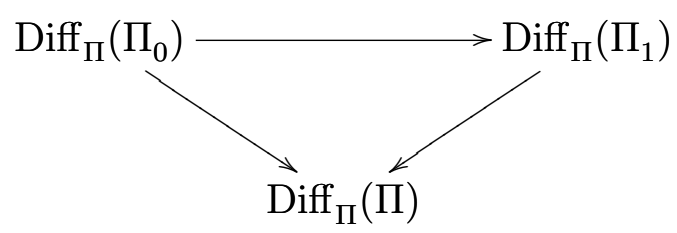

commutes, hence $\operatorname{Diff}_{\Pi}\left(\Pi_{0}\right) \longrightarrow \operatorname{Diff}_{\Pi}\left(\Pi_{1}\right)$ is a monomorphism of $\Pi$-modules.

Proof. ${ }^{1}$ We write $x \in \Pi_{0}, y \in \Pi$ and present an isomorphism

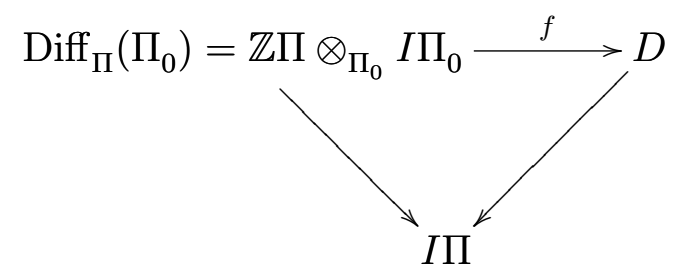

\footnotetext{
${ }^{1}$ There is a simple exact-sequences argument.
} 
where $D$ is the $\Pi$-submodule generated by all $x-1 . f$ and $f^{-1}$ are the $\Pi$-linear maps determined by the correspondence $1 \otimes(x-1) \Leftrightarrow x-1 . \quad f$ is more-or-less obviously well-defined. As for $f^{-1}$, it is deduced from the exact sequence of $\Pi$-modules

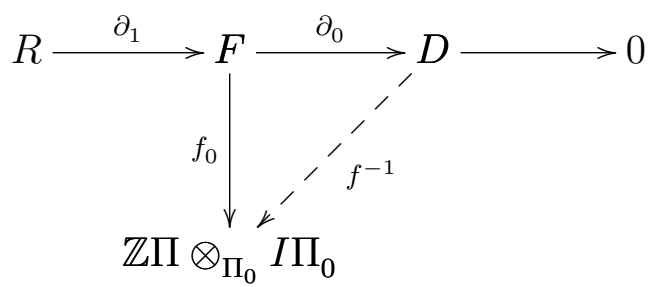

where $F$ is the free $\Pi$-module on generators $[x],[x] \partial_{0}=x-1$, and $\partial_{1}$ is the sub-module generated by all elements of the form

$$
y[x]+y_{1}\left[x_{1}\right]-y_{1}\left[y_{1}^{-1} y x\right]
$$

where $y=y_{1} x_{1} . f_{0}$ is defined by $[x] f_{0}=1 \otimes(x-1)$ and annihilates $\partial_{1}$.

For the proof of the statements around (b), we know that

$$
H_{p}\left(\operatorname{Diff}_{\Pi_{0}}\left(\Pi_{0} G^{n+1}\right)_{n \geq 0}\right)= \begin{cases}\operatorname{Diff}_{\Pi_{0}}\left(\Pi_{0}\right) & p=0 \\ 0 & p>0\end{cases}
$$

by (1.2). After tensoring over $\Pi_{0}$ with $\mathbb{Z} \Pi$, which is $\Pi_{0}$-projective since $\Pi_{0} \longrightarrow \Pi$ is a subgroup, we find that the homology becomes $\operatorname{Diff}_{\Pi}\left(\Pi_{0}\right)$ in dimension 0 and 0 in dimensions $>0$. This accounts for the two columns of 0's in (b). The fact that $\partial=0$ results from exactness and the above Lemma, which implies that $\operatorname{Diff}_{\Pi}\left(\Pi_{0}\right) \longrightarrow \operatorname{Diff}_{\Pi}\left(\Pi_{1}\right) \oplus \operatorname{Diff}_{\Pi}\left(\Pi_{2}\right)$ is monomorphic. This completes the proof.

(9.5) Commutative algebras. If

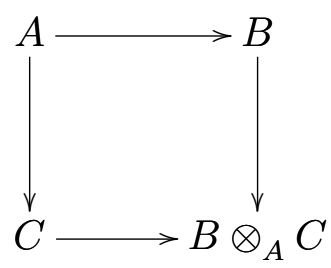

is a pushout in the category of commutative $K$-algebras, where $K$ is a commutative ring and $M$ is a $B \otimes_{A} C$-module, then

$$
H_{n}(A \longrightarrow B, M) \oplus H_{n}(A \longrightarrow C, M) \stackrel{\sim}{\longrightarrow} H_{n}\left(A \longrightarrow B \otimes_{A} C, M\right)
$$

for $n \geq 0$ if $\operatorname{Tor}_{p}^{A}(B, C)=0$ for $p>0$ (homology with respect to the absolute cotriple in the category of commutative $K$-algebras (cf. (7.4)). In this case (9.1) gives an exact sequence

$$
\cdots \rightarrow H_{n}(A, M) \rightarrow H_{n}(B, M) \oplus H_{n}(C, M) \rightarrow H_{n}\left(B \otimes_{A} C, M\right) \rightarrow H_{n-1}(A, M) \rightarrow \cdots
$$


A similar sequence holds in cohomology under the same Tor assumption. If $K=A$, this coincides with the homology coproduct theorem.

If $A \otimes_{K} B \longrightarrow C$ is a $K$-algebra map and $\operatorname{Tor}_{p}^{K}(A, B)=0$ for $p>0$, then the homology assumption in (9.2) is satisfied and we get the sequence

$$
\begin{aligned}
\cdots \longrightarrow H_{n}(C, M) \longrightarrow H_{n}(A \longrightarrow & C, M) \oplus H_{n}(B \longrightarrow C, M) \\
& \longrightarrow H_{n}\left(A \otimes_{K} B \longrightarrow C, M\right) \longrightarrow H_{n-1}(C, M) \longrightarrow \cdots
\end{aligned}
$$

if $M$ is a $C$-module; similarly in cohomology. This is the same sequence as in [André (1967)], Section 5, but the assumption $\operatorname{Tor}_{p}^{K}(C, C)=0, p>0$ employed there is seen to be superfluous.

\section{Cotriples and models}

For our purposes it is sufficient to consider a category with models to be a functor $\mathbf{M} \longrightarrow \mathbf{C}$ where $\mathbf{M}$ is discrete. The objects of $\mathbf{M}$ are known as the models. Many cotriples can be constructed in the following manner.

(10.1) Model-Induced Cotriple. If $X \in \mathbf{C}$ let

$$
X G=\star_{M} \underset{M \in \mathrm{M}}{\longrightarrow} X
$$

the coproduct indexed by all maps of model objects $M \longrightarrow X$.

We assume that such coproducts exist in $\mathbf{C}$, and write $M \longrightarrow X$ instead of $M I \longrightarrow X$ in order to avoid having to name $I: \mathbf{M} \longrightarrow \mathbf{C}$

Let $\langle x\rangle: M \longrightarrow X G$ denote the canonical map of the cofactor indexed by a map $x: M \longrightarrow X$. Then

$$
X G \stackrel{X \varepsilon}{\longrightarrow} X
$$

is the map such that $\langle x\rangle X \varepsilon=x$ for all $x: M \longrightarrow X, M \in \mathbf{M}$.

$$
X G \stackrel{X \delta}{\longrightarrow} X G G
$$

is the map such that $\langle x\rangle X \delta=\langle\langle x\rangle\rangle$ for all such $x$. (Since $\langle x\rangle: M \longrightarrow X G,\langle\langle x\rangle\rangle: M \rightarrow(X G) G$.) Both $\varepsilon$ and $\delta$ are natural transformations, and as

$$
\begin{aligned}
& \langle x\rangle X \delta . X G \delta=\langle\langle\langle x\rangle\rangle\rangle=\langle x\rangle X \delta . X \delta G \quad \text { and } \\
& \langle x\rangle X \delta . X G \varepsilon=\langle\langle x\rangle\rangle X G \varepsilon=\langle x\rangle=\langle\langle x\rangle\rangle X \varepsilon G=\langle x\rangle X \delta . X \varepsilon G
\end{aligned}
$$

we have that $\mathbf{G}=(\mathbf{G}, \varepsilon, \delta)$ is a cotriple in $\mathbf{C}$, which we call model-induced. (This special case is dual to the "triple structure" which Linton discusses in [Linton (1969)]; see also [Appelgate \& Tierney (1969)].)

If $M$ is a model, then $M$ viewed as an object in $\mathbf{C}$ is $\mathbf{G}$-projective (even a $\mathbf{G}$-coalgebra):

$$
M \stackrel{\langle M\rangle}{\longrightarrow} M G \stackrel{M \varepsilon}{\longrightarrow} M .
$$


Some other relations between model concepts and cotriple concepts are: A simplicial object $X_{*}$, has zero homotopy relative to $\mathbf{G}$ (every $\left(A G, X_{*}\right)$ has zero homotopy) $\Leftrightarrow$ every simplicial set $\left(M, X_{*}\right)$ has zero homotopy. In the additive case, $\mathbf{G}$-acyclicity is equivalent to acyclicity relative to all of the objects $M \in \mathbf{M}$.

(10.2) Examples of MOdel-InduCEd Cotriples. (a) Let $1 \longrightarrow R$-Mod be the functor whose value is $R$. Then $A G=\oplus R$, over all elements $R \rightarrow A$, is the free $R$-module cotriple (1.1). More generally, if $\mathbf{C}$ is tripleable over sets and $1 \rightarrow \mathbf{C}$ has value $1 F$, the free object on 1 generator, then the model-induced cotriple $\mathbf{G}$ is the free cotriple in $\mathbf{C}$, e.g., $\mathbf{C}=K$-Alg, $1 F=K[x], \mathbf{C}=$ Groups, $1 F=\mathbb{Z}$.

(b) Let $1 \longrightarrow$ Ab have value $\mathbb{Q} / \mathbb{Z}$ (rationals mod one). Let $T$ be the model-induced triple in $\mathrm{Ab}$

$$
A T=\prod_{A \longrightarrow \mathbb{Q} / \mathbb{Z}} \mathbb{Q} / \mathbb{Z} .
$$

$\left(A T^{n+1}\right)_{n \geq-1}$ is an injective resolution of $A$. The composition

$$
R-\operatorname{Mod} \longrightarrow \mathrm{Ab} \stackrel{T}{\longrightarrow} \mathrm{Ab} \stackrel{\mathrm{Hom}_{\mathbb{Z}}(R,-)}{\longrightarrow} R-\mathrm{Mod}
$$

is the Eckmann-Schopf triple $T_{R}$ in $R$-Mod. $\left(A T^{n+1}\right)_{n \geq-1}$ is an R-injective resolution of an $R$-module $A$.

(c) Let $\mathbf{M} \longrightarrow R$-Mod be the subset of cyclic $R$-modules. The model-induced cotriple is the pure cotriple

$$
C G=\bigoplus_{\substack{R / I \underset{I \subset R}{I \subset R} \\ I \neq 0}} R / I .
$$

The G-homology and cohomology of $C \in R$-Mod with coefficients in $A \otimes_{R}\left({ }_{-}\right)$, resp. $\operatorname{Hom}_{R}(-, A)$, are Harrison's $\operatorname{Ptor}_{n}^{R}(A, C)$, $\operatorname{Pext}_{R}^{n}(C, A)$; Pext classifies pure extensions of $R$-modules [Harrison (1959)]. This example is one of the original motivations for relative homological algebra.

(d) Let $\Delta \longrightarrow$ Top be the discrete subcategory whose objects are the standard Euclidian simplices $\Delta_{p}, p \geq 0$. Then

$$
X G=\bigcup_{\Delta_{p} \underset{p \geq 0}{\longrightarrow}{ }_{p \geq X}} \Delta_{p}
$$

If Top $\stackrel{E}{\longrightarrow} \mathscr{A}$ is $H_{0}(-, M)_{\text {sing }}$, the 0-th singular homology group of $X$ with coefficients in $M$, then

$$
H_{n}\left(X, H_{0}(-, M)_{\text {sing }}\right)_{\mathbf{G}} \simeq H_{n}(X, M)_{\text {sing }} .
$$

This is proved by a simple acyclic-models argument (11.2) or equivalently by collapsing of a spectral sequence like that in (10.5) . Singular cohomology is similarly captured.

(e) Let $\boldsymbol{\Delta} \longrightarrow$ Simp be the discrete subcategory of all $\Delta_{p}, p \geq 0$, where Simp is the category of simplicial spaces. The model-induced cotriple is $X G=\cup \Delta_{p}$ over all simplicial maps $\Delta_{p} \longrightarrow X, p \geq 0$. The $\mathbf{G}$-homology is simplicial homology. 
(10.3) Homology of A CAtegory. In [Roos (1961)], [André (1965)] Roos and André defined a homology theory $H_{n}(\mathbf{X}, E)$ of a category $\mathbf{X}$ with coefficients in a functor $E: \mathbf{X} \longrightarrow \mathscr{A}$. The homology theory arises from a complex

$$
C_{n}(\mathbf{X}, E)=\sum_{M_{0} \stackrel{\alpha_{0}}{\longrightarrow} M_{1} \longrightarrow \ldots \stackrel{\alpha_{n-1}}{\longrightarrow} M_{n}} M_{0} E, \quad n \geq 0 .
$$

Using the \langle\rangle notation for the coproduct injections $M_{0} E \longrightarrow C_{n}(\mathbf{X}, E)$, the face operators $\varepsilon_{i}: C_{n} \longrightarrow C_{n-1}, 0 \leq i \leq n$, are

$$
\left\langle\alpha_{0}, \ldots, \alpha_{n-1}\right\rangle \varepsilon_{i}= \begin{cases}\alpha_{0} E .\left\langle\alpha_{1}, \ldots, \alpha_{n-1}\right\rangle, & i=0 \\ \left\langle\alpha_{0}, \ldots, \alpha_{i-1} \alpha_{i}, \ldots, \alpha_{n-1}\right\rangle, & 0<i<n \\ \left\langle\alpha_{0}, \ldots, \alpha_{n-2}\right\rangle, & i=n\end{cases}
$$

it is understood that $\left\langle\alpha_{0}\right\rangle \varepsilon_{0}=\alpha_{0} E \cdot\left\langle M_{1}\right\rangle,\left\langle\alpha_{0}\right\rangle \varepsilon_{1}=\left\langle M_{0}\right\rangle$ and $C_{0}(\mathbf{X}, E)=\sum M E$ over all $M \in \mathbf{X}$. The homology groups of this complex, with respect to the boundary operator $\partial=\sum(-1)^{i} \varepsilon_{i}$, are denoted by $H_{n}(\mathbf{X}, E)$.

Clearly, $H_{0}(\mathbf{X}, E)=\lim E$, and Roos proves that if $\mathscr{A}$ has exact direct sums (AB4), then $H_{n}(\mathbf{X}, E)=\left(L_{n} \lim _{\rightarrow} \overrightarrow{)}(E)\right.$, the left satellite of the direct limit functor $(\mathbf{X}, \mathscr{A}) \longrightarrow \mathscr{A}$, for $n>0$.

If there is a terminal object $1 \in \mathbf{X}$, then $H_{n}(\mathbf{X}, E)=0$ for $n>0$. This follows from the existence of homotopy operators

$$
C_{0} \stackrel{h_{0}}{\longrightarrow} C_{1} \longrightarrow \cdots \rightarrow C_{n} \stackrel{h_{n}}{\longrightarrow} \cdots
$$

defined by $\left\langle\alpha_{0}, \ldots, \alpha_{n}\right\rangle h_{n}=\left\langle\alpha_{0}, \ldots, \alpha_{n},(-)\right\rangle$ where (-) is the unique map of the appropriate object into 1 . This is also obvious from the fact that $\lim _{\rightarrow} E=1 E$, that $\lim _{\rightarrow}$ is an exact functor (assuming $\mathscr{A}$ is AB4).

More generally, if $\mathbf{X}$ is directed and $\mathscr{A}$ is AB5, then $H_{n}(\mathbf{X}, E)=0$ for $n>0$. "Directed" means that if $X_{0}, X_{1} \in \mathbf{X}$, then there exist an object $X \in \mathbf{X}$, maps $X_{0} \longrightarrow X \longleftarrow X_{1}$, and if $x, y: X_{1} \longrightarrow X_{0}$, then there exists a map $z: X_{0} \longrightarrow X$, such that $x z=y z$. AB5 is equivalent to exactness of direct limits over directed index categories.

(10.4) André-Appelgate homology. In a models situation, let Im $\mathbf{M}$ be the full subcategory of $\mathbf{C}$ generated by the image of $\mathbf{M} \longrightarrow \mathbf{C}$. If $X \in \mathbf{C},(\operatorname{Im} \mathbf{M}, X)$ is the category whose objects are maps of models $M \longrightarrow X$ and whose maps are triangles $X \longleftarrow M_{0} \longrightarrow M_{1} \longrightarrow X$. If $E_{0}: \operatorname{Im} \mathbf{M} \longrightarrow \mathscr{A}$ is a coefficient functor, $E_{0}$ can be construed as a functor $(\operatorname{Im} \mathbf{M}, X) \longrightarrow \mathscr{A}$ by $(M \longrightarrow X) E_{0}=M E_{0}$.

The André-Appelgate homology of $X$ with coefficients in $E_{0}$ (relative to the models $\mathbf{M} \rightarrow \mathbf{C})$ is

$$
A_{n}\left(X, E_{0}\right)=H_{n}\left[(\operatorname{Im~M}, X), E_{0}\right]
$$


where on the right we have the Roos-André homology of the comma category. Explicitly, the chain complex which gives rise to this homology theory has

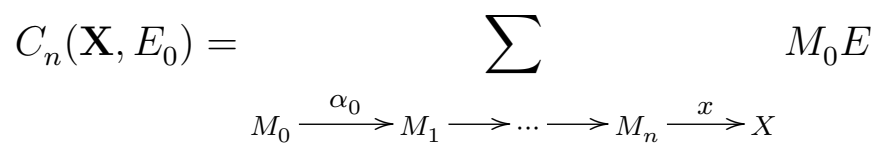

with boundary operator as in [André (1967)], Section 1. We note that H. Appelgate [Appelgate (1965)] developed this homology theory in a different way. He viewed the above complex as being generated by its 0-chains acting as a cotriple in the functor category $(\mathbf{C}, \mathscr{A})$.

A basic property of this theory is that if $\mathrm{M}$ is a model, then

$$
A_{n}\left(M, E_{0}\right) \simeq \begin{cases}M E_{0}, & n=0 \\ 0, & n>0,\end{cases}
$$

for any functor $E_{0}: \operatorname{Im} \mathbf{M} \longrightarrow \mathscr{A}$. The category $(\operatorname{Im} \mathbf{M}, M)$ has $M$ as final object and the contracting homotopy in (10.3) in available [André (1967)], Sub-section 1.1.

In general,

$$
A_{0}\left(X, E_{0}\right)=X . E_{J}\left(E_{0}\right)
$$

where $E_{J}:(\operatorname{Im} \mathbf{M}, \mathscr{A}) \longrightarrow(\mathbf{C}, \mathscr{A})$, the Kan extension, is left adjoint to the restriction functor $(\operatorname{Im} \mathbf{M}, \mathscr{A}) \longleftarrow(\mathbf{C}, \mathscr{A})$. As the Kan extension can also be written as $\lim _{\rightarrow}\left(E_{0}:(\operatorname{Im} \mathbf{M}, X) \longrightarrow \mathscr{A}\right)$, Roos's result implies that

$$
A_{n}\left(X, E_{0}\right)=X .\left(L_{n} E_{J}\right)\left(E_{0}\right), \quad n>0 .
$$

provided that $\mathscr{A}$ is AB4. (For further information about Kan extension, see Ulmer's paper in this volume.)

The theory $A_{n}(X, E)$ is also defined when $E: \mathbf{C} \longrightarrow \mathscr{A}$ by restricting $E$ to Im $\mathbf{M}$. It can always be assumed that the coefficient functor is defined on all of $\mathbf{C}$. If not, take the Kan extension. The restriction of $E_{J}\left(E_{0}\right)$ to $\operatorname{Im} \mathbf{M}$ is equivalent to the given $E_{0}$ since $J: \operatorname{Im} \mathbf{M} \longrightarrow \mathbf{C}$ is full.

Now suppose we have both a models situation $\mathbf{M} \longrightarrow \mathbf{C}$ and a cotriple $\mathbf{G}$ in $\mathbf{C}$. To compare the homology theories $A_{n}(X, E)$ and $H_{n}(X, E)=H_{n}(X, E)_{\mathbf{G}}$, we use:

(10.5) Spectral Sequence. Suppose that all models $M \in \mathbf{M}$ are $\mathbf{G}$-projective. Then there is a spectral sequence

$$
H_{p}\left(X, A_{q}(-, E)\right) \Longrightarrow A_{p+q}(X, E)
$$

where the total homology is filtered by levels $\leq p$.

Proof. For each $M \in \mathbf{M}$ choose a map $M \sigma: M \longrightarrow M G$ such that $M \sigma \cdot M \varepsilon=M$. Define $\vartheta_{q}: C_{q}(X, E) \longrightarrow C_{q}(X G, E)$ by the identity map from the $\left\langle\alpha_{0}, \ldots, \alpha_{q-1}, x\right\rangle$-th summand to the $\left\langle\alpha_{0}, \ldots, \alpha_{q-1} \cdot M_{q} \sigma, x G\right\rangle$-th. This makes $C_{*}(X, E) \mathbf{G}$-representable, and the result follows from (11.3). 
Proposition (10.6). If each category (Im M,XG) is directed and the coefficient category $\mathscr{A}$ is $A B 5$, then the above spectral sequence collapses and gives edge isomorphisms

$$
H_{n}\left(X, A_{0}(-, E)\right) \stackrel{\sim}{\longrightarrow} A_{n}(X, E), \quad n>0 .
$$

The André-Appelgate theory has a natural augmentation $A_{0}(-, E) \longrightarrow E$, which is induced by the following cokernel diagram and map $e$ such that $\langle x\rangle e=x E$ :

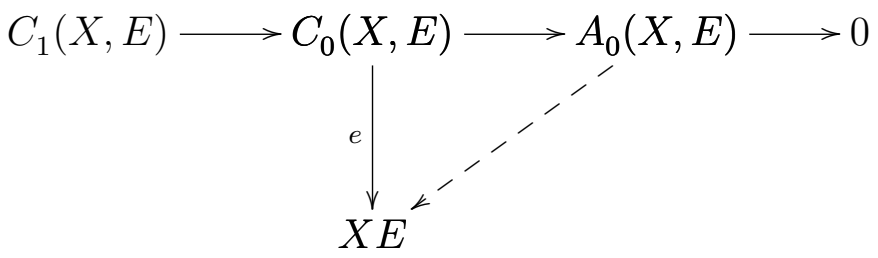

We obtain isomorphisms $H_{n} \simeq A_{n}$ from (10.6) when the augmentation is an isomorphism. Proposition (10.7). Equivalent are:
(1) $A_{0}(-, E) \longrightarrow E$
is an isomorphism,
(2) $E=E_{J}\left(E_{0}\right)$, where $E_{0}: \operatorname{Im} \mathbf{M} \longrightarrow \mathscr{A}$
(3) $\quad E=E_{J}(E)$ the Kan extension of $E$ restricted to $\operatorname{Im} \mathbf{M}$.

Finally, (1) (2) (3) are implied by:

(4) $E$ commutes with direct limits and $\mathrm{Im} \mathbf{M} \longrightarrow \mathbf{C}$ is adequate [Isbell (1964)] /dense [Ulmer (1968)].

The equivalences are trivial in view of fullness of $J: \operatorname{Im} \mathbf{M} \longrightarrow \mathbf{C}$. As to (4), this results from the fact that $J$ is adequate/dense $\Leftrightarrow \lim _{\rightarrow}[(\operatorname{Im} \mathbf{M}, X) \longrightarrow \mathbf{C}]=X$ for all $X \in \mathbf{C}$

(10.8) Examples in Which the models are G-PRojective. (a) Let the models be the values of the cotriple, that is, all $X G, X \in \mathbf{C}$. The comma category $(\operatorname{Im} \mathbf{M}, X G)$ has $X G$ as terminal object, hence is directed. Thus $A_{q}(X G, E)=0$ for $q>0$ and any $E$, and (10.6) gives an isomorphism

$$
H_{n}\left(X A_{0}(-, E)\right) \stackrel{\sim}{\longrightarrow} A_{n}(X, E), \quad n \geq 0 .
$$

(10.7) is inapplicable in general.

A stronger result follows directly from acyclic models $(11.2)$. The complex $C_{*}(X, E)$ is $\mathbf{G}$-representable (10.5) and is $\mathbf{G}$-acyclic since each $X G$ is a model. Thus

$$
H_{n}(X, E) \stackrel{\sim}{\longrightarrow} A_{n}(X, E)
$$

(b) Another convenient set of models with the same properties is that of all $\mathbf{G}$ projectives.

Here and above, existence of the André-Appelgate complex raises some difficulties. The sets of models are too large. However, for coefficient functors with values in AB5 
categories with generators, the problem can be avoided. Such categories are Ab-topos [Roos (1961)], realizable as categories of abelian sheaves on suitable sites, and it suffices to pass to models or abelian groups in a larger universe. (See the discussion of this point in [André (1967)] as well.)

(c) Let $\mathbf{G}$ be the free $R$-module cotriple and let $\mathbf{M} \longrightarrow R$-Mod be the set of finitely generated free $R$-modules. The categories ( $\operatorname{Im} \mathbf{M}, X G)$ are directed, since any $M \longrightarrow X G$ can be factored

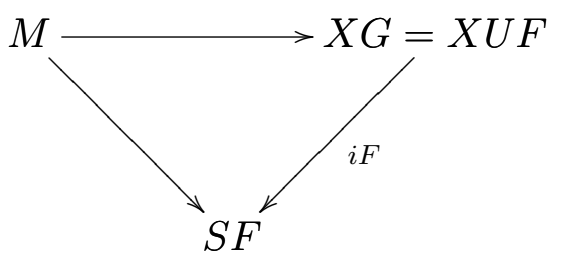

where $i: S \longrightarrow X U$ is a finite subset of the free basis $X U$, ( $U$ is the underlying set functor.) Moreover, $\operatorname{Im} \mathbf{M} \longrightarrow R$-Mod is adequate. Thus if $E$ is any cocontinuous coefficient functor with values in an AB5 category, then $H_{n}(X, E) \simeq A_{n}(X, E)$ for all $R$-modules $X$.

(d) More generally, if $\mathbf{C}$ is tripleable over sets, $\aleph$ is a rank of the triple [Linton (1966a)] and $\mathbf{M}$ is the set of free algebras on fewer than $\aleph$ generators, then $(\operatorname{Im} \mathbf{M}, X G)$ can be proved directed in the same way, Thus homology relative to the models agrees with the cotriple homology (for cocontinuous AB5-category-valued coefficient functors; $\mathbf{G}$ is the free algebra cotriple relative to sets).

In these examples, adequacy/denseness of $\operatorname{Im} \mathbf{M}$ is well known or easily verified. In the following case adequacy fails. Let $1 \longrightarrow R$-Mod have $R$ as value. $A_{0}(X, i d$. $) \longrightarrow X$ is non-isomorphic (coefficients are in the identity functor $R$-Mod $\longrightarrow R$-Mod). In fact $A_{0}(X, i d)=.R(X) / I$, the free $R$-module on $X$ modulo the submodule generated by all $r(x)-(r x)$. Of course, $H_{0}(X, i d$. $) \simeq X$ (homology with respect to the absolute cotriple, which is induced by the above model).

(e) Cohomology. Let $E: \mathbf{C} \longrightarrow \mathscr{A}^{*}$ be a "contravariant" coefficient functor. Isomorphisms $A^{n}(X, E) \stackrel{\sim}{\longrightarrow} H^{n}(X, E)$ follow purely formally in cases (a), (b) above. Cases (c), (d) offer the difficulty that the coefficient category $\mathscr{A}^{*}$ cannot be assumed to be AB5, since in practice it is usually dual to a category of modules and therefore AB $5^{*}$. Assume that the rank $\aleph$ of the triple $\mathbf{T}$ is $\aleph_{0}$, however, one can proceed as follows.

If $X$ is a $\mathbf{T}$-algebra, the category of $X$-modules is abelian, AB5, has a projective generator and is complete and cocomplete. Thus injective resolutions can be constructed, in the abelian category sense. Moreover, the free abelian group functor $\operatorname{Diff}_{X}:(\mathbf{C}, X) \longrightarrow X$-Mod exists. Consider the André-Appelgate complex with values in $X$-Mod: $\left(C_{p}(X)\right)=\left(C_{p}\left(X, \operatorname{Diff}_{X}\right)_{p \geq 0}\right)$. Its homology, written $A_{p}(X)$, measures the failure of the André-Appelgate theory to be a derived functor on the category $X$-Mod. If $Y \longrightarrow X$ is an $X$-module, $\left(\operatorname{Hom}_{X}\left(C_{p} X, Y\right)_{p \geq 0}\right)$ has $A^{P}(X, Y)$ as its cohomology. Let $\left(Y^{q}\right)_{q \geq 0}$ be an injective resolution of $Y$. We get a double complex $\left(\operatorname{Hom}_{X}\left(C_{p} X, Y^{q}\right)_{p, q \geq 0}\right)$, hence a universal-coefficients spectral sequence

$$
\operatorname{Ext}_{X}^{q}\left(A_{p}(X), Y\right) \longrightarrow A^{p+q}(X, Y)
$$


where the total cohomology is filtered by $q$. (Use the fact that the complex $C_{p}(X)$ consists of projective $X$-modules.)

For example, in the case of commutative $A$-algebras over $B$, one obtains

$$
\operatorname{Ext}_{B}^{q}\left(H_{p}(A, B, B), M\right) \longrightarrow H^{p+q}(A, B, M)
$$

in the notation of [André (1967)], Section 16.

Similarly, in the cotriple theory, there is a spectral sequence

$$
\operatorname{Ext}_{X}^{q}\left(H_{p}(X), Y\right) \longrightarrow H^{p+q}(X, Y)
$$

Now, by the assumption that the rank of the triple is $\aleph_{0}$, the free $\mathbf{T}$-algebra $X G \longrightarrow X$ is a filtered direct limit of free $\mathbf{T}$-algebras of finite type, that is, of models. Since the homology $A_{p}(-)$ commutes with filtered limits, $A_{p}(X G)=0$ for $p>0, A_{0}(X G)=\operatorname{Diff}_{X}(X G)$. Thus the above spectral sequence yields $A^{n}(X G, Y)=0$ for $n>o, A^{0}(X G, Y)=$ $\operatorname{Hom}_{X}(X G, Y)$. Acyclic models (11.2) now yields isomorphisms

$$
A^{n}(X, Y) \stackrel{\sim}{\longrightarrow} H^{n}(X, Y) .
$$

A case in which this comparison technique runs into difficulty is the following. Let $\mathbf{M} \longrightarrow K$-Alg be the set of tensor algebras of finitely generated $K$-modules, and let $\mathbf{G}$ be the cotriple in $K$-Alg relative to $K$-modules. Homology isomorphisms $H_{n} \stackrel{\sim}{\longrightarrow} A_{n}$ are easily obtained, as in (d). But the above derivation of the universal-coefficients spectral sequence does not work, because one seems to need to resolve the module variable both $\mathscr{S}$-relatively and $K$-relatively at the same time.

\section{Appendix on acyclic models}

Let $0 \longleftarrow C_{-1} \longleftarrow C_{0} \longleftarrow C_{1} \longleftarrow \cdots$ be a chain complex of functors $\mathbf{C} \longrightarrow \mathscr{A}$. $\left(C_{n}\right)$ is $\mathbf{G}$-representable, where $\mathbf{G}$ is a cotriple in $\mathbf{C}$, if there are natural transformations $\vartheta_{n}: C_{n} \longrightarrow G C_{n}$ such that $\vartheta_{n} . \varepsilon C_{n}=C_{n}$ for all $n \geq 0 . \quad\left(C_{n}\right)$ is G-contractible if the complex $\left(G C_{n}\right)_{n \geq-1}$ has a contracting homotopy (by natural transformations).

Proposition [(11.1)]. [Barr \& Beck (1966)] Suppose that $\left(C_{n}\right)$ is $\mathbf{G}$-representabie, $\left(K_{n}\right)$ is $\mathbf{G}$-contractible, and $\varphi_{-1}: C_{-1} \longrightarrow K_{-1}$ is a given natural transformation, then $\varphi_{-1}$ can be extended to a natural chain transformation $\left(\varphi_{n}\right):\left(C_{n}\right) \longrightarrow\left(K_{n}\right)_{n \geq-1}$ by the inductive formula

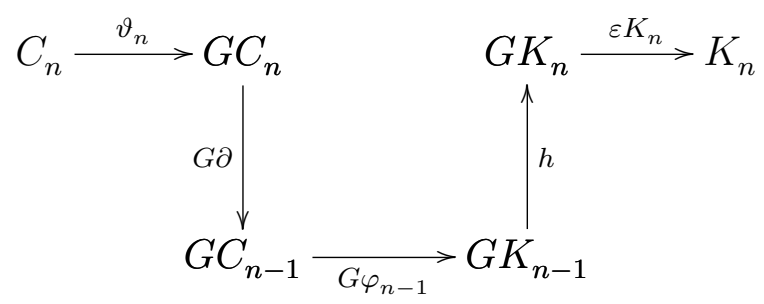

Any two extensions of $\varphi_{-1}$ are naturally chain homotopic (we omit the formula). 
In particular, if $C_{-1}=K_{-1}$, then there are natural chain equivalences $\left(C_{n}\right) \stackrel{\sim}{\rightleftarrows}\left(K_{n}\right)$. If $E: \mathbf{C} \longrightarrow \mathscr{A}$ is a functor with values in an additive category, then the standard chain complex

$$
0 \longleftarrow E \longleftarrow G E \longleftarrow G^{2} E \longleftarrow \cdots
$$

is $\mathbf{G}$-representable and $\mathbf{G}$-contractible by virtue of $\partial G^{n} E: G^{n+1} E \longrightarrow G^{n+2} E$. Thus if

$$
0 \longleftarrow E \longleftarrow E_{0} \longleftarrow E_{1} \longleftarrow \cdots
$$

is any $\mathbf{G}$-representable chain complex of functors $\mathbf{C} \longrightarrow \mathscr{A}$, there exists a unique natural chain transformation $\left(\varphi_{n}\right):\left(E_{n}\right) \longrightarrow\left(G^{n+1} E\right)$ such that $\varphi_{-1}=E$ (up to homotopy).

The proof is more of less contained in the statement. The term "G-contractible" was not used in [Barr \& Beck (1966)], the term "G-acyclic" used there is reintroduced below with a different meaning.

The conclusions of (11.1) in practice are often too hard to establish and too strong to be relevant, At present all we need is homology isomorphism - a conclusion which is much weaker than chain equivalence. Thus it is convenient and reasonably satisfying to have the following weaker result available (as M. André has pointed out to us - see also [André (1967)]), that one can conclude a homology isomorphism $H\left(X E_{*}\right) \longrightarrow H(X, E)_{\mathbf{G}}$ from the information that the complex $E_{*}$ is $\mathbf{G}$-representable as above and $\mathbf{G}$-acyclic merely in the sense that $H_{n}\left(X G E_{*}\right)=0$ if $n>0$, and $=X G E$ if $n=0$. This observation greatly simplifies proofs of agreement between homology theories arising from standard complexes, such as those of [Barr \& Beck (1966)].

Proposition (11.2). Let

$$
0 \longleftarrow E \longleftarrow E_{0} \longleftarrow E_{1} \longleftarrow \cdots
$$

be a complex of functors $\mathbf{C} \longrightarrow \mathscr{A}$ such that

$$
H_{n}\left(X G E_{*}\right)= \begin{cases}X G E, & n=0 \\ 0 & n>0\end{cases}
$$

and the $\mathbf{G}$-homology groups

$$
H_{p}\left(X, E_{q}\right)_{\mathbf{G}}= \begin{cases}X E_{q}, & p=0 \\ 0, & p>0,\end{cases}
$$

for all $X \in \mathbf{C}, q \geq 0$. Then the spectral sequences obtained from the double complex

$$
\left(X G^{p+1} E_{q}\right)_{p, q \geq 0}
$$

by filtering by levels $\leq p$ and $\leq q$ both collapse, giving edge isomorphisms

$$
\begin{array}{ll}
H_{n}\left(X E_{*}\right) \stackrel{\sim}{\longrightarrow} \text { total } H_{n} & (p \text { filtration }) \\
H_{n}(X, E)_{\mathbf{G}} \stackrel{\sim}{\longrightarrow} \text { total } H_{n} & \text { (q filtration) }
\end{array}
$$


for all $n \geq 0$, hence natural isomorphisms $H_{n}\left(X E_{*}\right) \stackrel{\sim}{\longrightarrow} H_{n}(X, E)_{\mathbf{G}}$.

In particular, G-representability of the complex $\left(E_{n}\right)$ guarantees the second acyclicity condition, since the $E_{q}$ are then retracts of the $\mathbf{G}$-acyclic functors $G E_{q}, q \geq 0$.

There is an obvious overlap between these two propositions which we encountered in Theorem (8.1):

Proposition (11.3). Let $0 \longleftarrow E \longleftarrow E_{0} \longleftarrow \cdots$ be a $\mathbf{G}$-representable chain complex of functors $\mathbf{C} \longrightarrow \mathscr{A}$ and $\left(\varphi_{n}\right):\left(E_{n}\right) \longrightarrow\left(G^{n+1} E\right), n \geq-1$, a chain transformation such that $\varphi_{-1}=E($ see (11.1)). By $\mathbf{G}$-representability, the acyclicity hypothesis

$$
H_{p}\left(X, E_{q}\right)= \begin{cases}X E_{q}, & p=0 \\ 0, & p>0\end{cases}
$$

is satisfied and the rows of the double complex $X G^{p+1} E_{q}$ have homology zero. We obtain a spectral sequence

$$
H_{p}\left(X, H_{q}\left(-, E_{*}\right)\right)_{G} \longrightarrow H_{p+q}\left(X E_{*}\right),
$$

where the total homology is filtered by levels $\leq p$. The edge homomorphisms are

$$
H_{0}\left(X, H_{n}\left(-, E_{*}\right)\right)_{\mathbf{G}} \stackrel{\lambda_{\mathbf{G}}}{\longrightarrow} H_{n}\left(X E_{*}\right)
$$

and the top map in the commutative diagram

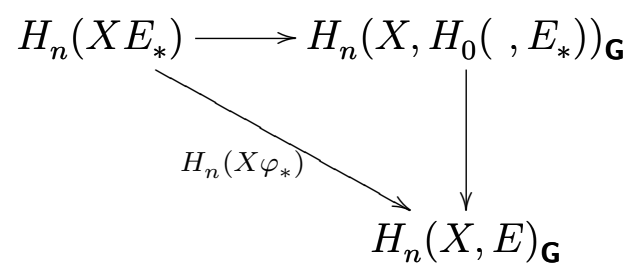

Finally suppose that

$$
H_{n}\left(X G E_{*}\right)= \begin{cases}X G E, & n=0 \\ 0 & n>0 .\end{cases}
$$

The spectral sequence collapses, as $H_{p}\left(X, H_{q}\left(-, E_{*}\right)\right)_{\mathbf{G}}=0$ if $q>0$. The edge homomorphism $\lambda_{\mathbf{G}}$ is zero. The second edge homomorphism and the vertical map in the above triangle both become isomorphisms. Thus the homology isomorphism produced by (11.2) is actually induced by the chain map $\varphi_{*}: E_{*} \longrightarrow\left(G^{n+1} E\right)_{n \geq 0}$.

The proof is left to the reader.

\section{References}

[André (1965)] M. André,Limites et fibrés. C. R. Acad. Sci. 260 (1965), 756-759. 
[André (1967)] M. André, Méthode simpliciale en algèbre homologique et algèbre commutative. Lecture Notes Math. 32 (1967), Springer-Verlag, Berlin.

[Appelgate (1965)] H. Appelgate, Acyclic models and resolvent functors. Dissertation, Columbia University, New York (1965).

[Appelgate \& Tierney (1969)] H. Appelgate \& M. Tierney, Categories with models. This volume.

[Barr (1966)] M. Barr, Cohomology in tensored categories. Proc. Conf. Categorical Algebra (La Jolla, 1965). Springer-Verlag, Berlin (1966), 344-354.

[Barr (1967)] M. Barr, Shukla cohomology and triples. J. Alg. 5 (1967), 222-231.

[Barr (1969)] M. Barr, Composite cotriples and derived functors. This volume.

[Barr (1968)] M. Barr, Harrison homology, Hochschild homology and triples. J. Alg. 8 (1968), 314-323.

[Barr \& Beck (1966)] M. Barr \& J. Beck, Acyclic models and triples. Proc. Conf. Categorical Algebra (La Jolla, 1965). Springer-Verlag, Berlin (1966), 336-343.

[Barr \& Rinehart (1966)] M. Barr \& G. Rinehart, Cohomology as the derived functor of derivations. Trans. Amer. Math. Soc. 122 (1966), 416-426.

[Beck (1967)] J. Beck, Triples, algebras, and cohomology. Dissertation, Columbia University, New York (1967). Republished electronically, Reprinted as Theory and Applications of Categories, 2 (2003), http://www.tac.mta.ca/tac/reprints/articles/2/tr2abs.html

[Cartan \& Eilenberg (1956)] H. Cartan \& S. Eilenberg, Homological Algebra. Princeton University Press (1956).

[Eckmann \& Stammbach (1967)] B. Eckmann \& U. Stammbach , Homologie et différentielles. C. R. Acad. Sci. 265 (1967), 46-48.

[Eilenberg \& Moore (1965)] S. Eilenberg \& J. C. Moore, Foundation of relative homological algebra. Memoirs Amer. Math. Soc. 55, (1965).

[Eilenberg \& Steenrod (1952)] S. Eilenberg \& N. Steenrod, Foundations of Algebraic Topology. Princeton Univerity Press (1952).

[Eilenberg \& Zilber (1953)] S. Eilenberg \& J. A. Zilber, On products of complexes. Ann. Math. 75 (1953), 200-204.

[Freyd (1964)] P. J. Freyd, Abelian Categories. Harper \& Row, New York (1964). Republished electronically, Reprints in Theory and Applications of Categories, 3 (2003), http://www.tac.mta. $\mathrm{ca} / \mathrm{tac} / \mathrm{reprints/articles/3/tr3abs.html}$

[Grothendieck \& Dieudonné (1964)] A. Grothendieck \& J. Dieudonné, Éléments de géométrie algébrique: IV. Étude locale des schémas et des morphismes de schémas. Publ. Math. IHÉS 20 (1964). Available online at http://www.numdam.org/item?id=PMIHES_1964_20_5_0

[Harrison (1959)] D. K. Harrison, Infinite abelian groups and homological methods. Ann. Math. 69 (1959), 366-391.

[Harrison (1962)] D. K. Harrison, Commutative algebras and cohomology. Trans. Amer. Math. Soc. 104 (1962), 191-204. 
[Hochschild (1956)] G. Hochschild, Relative homological algebra. Trans. Amer. Math. Soc. 82 (1956), $246-269$.

[Huber (1961)] P. J. Huber, Homotopy theory in general categories. Math. Ann. 144 (1961), 361-385.

[Huber (1962)] P. J. Huber, Standard constructions in abelian categories. Math. Ann. 146 (1962), 321325.

[Isbell (1964)] J. R. Isbell, Subobjects, adequacy, completeness and categories of algebras. Rozprawy Mat. 36 (1964), Warszawa.

[Kleisli (1967)] H. Kleisli, Résolutions dans les catégories avec multiplication. C. R. Acad. Sci. 264 (1967), $11-14$.

[Lichtenbaum \& Schlessinger (1967)] S. Lichtenbaum \& M. Schlessinger, The cotangent complex of a morphism. Trans. Amer. Math. Soc. 128 (1967), 41-70.

[Linton (1966a)] F. E. J. Linton, Some aspects of equational categories. Proc. Conf. Categorical Algebra (La Jolla, 1965). Springer-Verlag, Berlin , (1966) 84-94.

[Linton (1969a)] F. E. J. Linton, Applied functorial semantics II. , Homology and standard constructions. Lecture Notes Math. 80. Reprinted in Theorem and Applications of Categories, 18 (1969), http:/ /www.tac.mta.ca/tac/reprints/articles/18/tr18.pdf .

[Linton (1969)] F. E. J. Linton, An Outline of functorial semantics. Homology and standard constructions. Lecture Notes Math. 80. Reprinted in Theorem and Applications of Categories, 18 (1969), http://www.tac.mta.ca/tac/reprints/articles/18/tr18.pdf .

[Mac Lane (1963)] S. Mac Lane, Homology. Springer-Verlag, Berlin (1963).

[Moore (1956)] J. C. Moore, Seminar on algebraic homotopy theory. Mimeographed, Princeton University (1956).

[Quillen (1967)] D. Quillen On the (co-) homology of commutative rings. Applications of Categorical Algebra (Proc. Sympos. Pure Math., Vol. XVII, New York, 1968). Amer. Math. Soc., Providence, R.I, 65-87.

[Ribes (1967)] L. Ribes, A cohomology theory for pairs of groups. Dissertation, University of Rochester (1967).

[Röhrl (1962)] H. Röhrl, Über satelliten halbexakter Funktoren. Math. Z. 79 (1962), 193-223.

[Roos (1961)] J.-E. Roos, Sur les foncteurs dérivés de lim . C. R. Acad. Sci. 252 (1961), 3702-3704.

[Shukla (1961)] U. Shukla, Cohomologie des algèbres associatives. Ann. Sci. École Norm. Sup. 78 (1961), 163-209.

[Stallings (1965)] J. Stallings, Homology and central series of groups. J. Alg. 2 (1965), 170-181.

[Stammbach (1966)] U. Stammbach, Anwendungen der Homologietheorie der Gruppen. Math. Z. 94 (1966), 157-177.

[Takasu (1959/60)] S. Takasu, Relative homology and relative cohomology of groups. J. Fac. Sci. Tokyo, Sec. I 8 (1959/60), 75-110. 
[Trotter (1962)] H. F. Trotter, Homology of group systems with applications to knot theory. Ann. Math. 76 (1962), 464-498.

[Ulmer (1968)] F. Ulmer, On cotriple and André (co)homology, their relationship with classical homological algebra. Homology and standard constructions. Lecture Notes Math. 80. Reprinted in Theorem and Applications of Categories, 18 (1968), http://www.tac.mta.ca/tac/reprints/articles/ 18/tr18.pdf .

[Wall (1966)] C. T. C. Wall, On the exactness of interlocking sequences. Enseignement Math. 12 (1966), 95-100. 